\title{
Configurationally Stabilized Analogs of M. ulcerans Exotoxins Mycolactones A and B reveal the Importance of Side Chain Geometry for Mycolactone Virulence
}

\author{
Matthias Gehringer, ${ }^{\mathrm{a}, \mathrm{c}}$ Patrick Mäder, ${ }^{\mathrm{ad}}$ Philipp Gersbach,, \\ Bernhard Pfeiffer, ${ }^{a}$ Nicole Scherr, ${ }^{\mathrm{b}, \mathrm{f}}$ Jean-Pierre Dangy, \\ Gerd Pluschke, ${ }^{\mathrm{c}}$ and Karl-Heinz Altmann ${ }^{\mathrm{a}, *}$
}

\section{Supporting Information}

${ }^{a}$ Swiss Federal Institute of Technology (ETH) Zürich

Department of Chemistry and Applied Biosciences

Institute of Pharmaceutical Sciences

Vladimir-Prelog-Weg 4, CH-8093 Zürich, Switzerland

E-mail: karl-heinz.altmann@pharma.ethz.ch

${ }^{\mathrm{b}}$ Swiss Tropical and Public Health Institute

Socinstrasse 57, CH-4002 Basel, Switzerland

${ }^{c}$ Current address:

Eberhard Karls Universität Tübingen

Institute of Pharmaceutical Sciences

Pharmaceutical and Medicinal Chemistry

Auf der Morgenstelle 8, D-72076 Tübingen, Germany

${ }^{\mathrm{d}}$ Current address:

ENDOTHERM GmbHScience-Park II

D-66123 Saarbrücken, Germany

${ }^{\mathrm{e}}$ Current address:

Akorn, Inc.

CH-8442 Hettlingen, Switzerland

${ }^{\mathrm{f}}$ Current address:

Biozentrum, University of Basel

Klingelbergstrasse 50/70, CH-4056 Basel, Switzerland 


\section{Content}

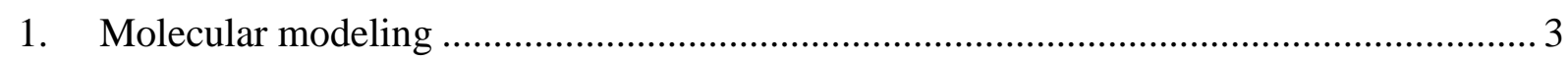

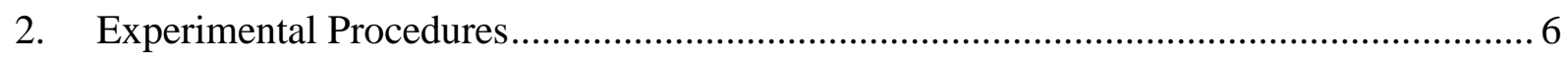

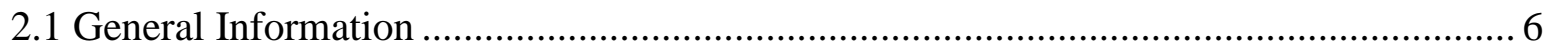

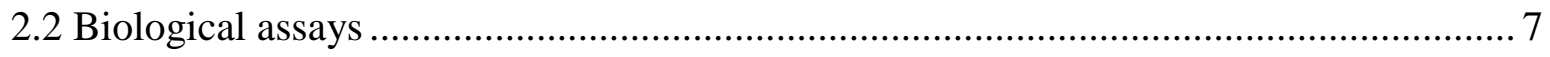

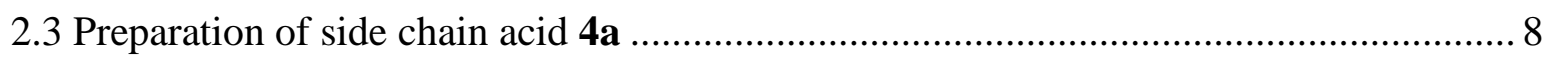

2.3.1 Synthesis of the western 1-phenyl-1H-tetrazol-5-yl sulfone building block 7 ........ 8

2.3.2 Assembly of sulfone $\mathbf{7}$ and aldehyde $\mathbf{8}$ by Julia-Kocienski olefination and ester

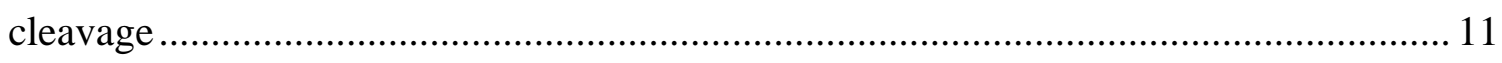

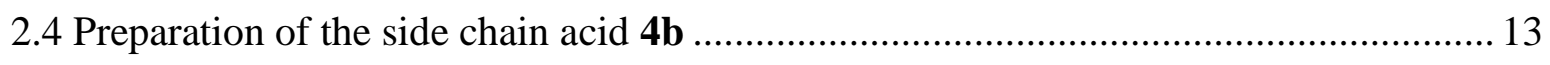

2.4.1 Synthesis of the western vinyl iodide building block 18 ................................ 13

2.4.2 Synthesis of the eastern vinyl stannane building block 14.............................. 17

2.4.3 Assembly of building blocks $\mathbf{1 2}$ and $\mathbf{1 4}$ by Stille coupling and ester cleavage ...... 18

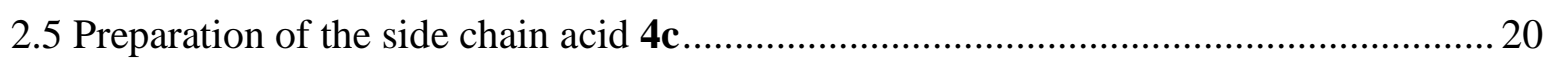

2.5.1 Synthesis of the western vinyl iodide building block 18 ................................ 20

2.5.2 Assembly of the building blocks $\mathbf{1 8}$ and $\mathbf{1 4}$ by Stille coupling and ester cleavage 25

2.6 Esterification of the mycolactone core with acids $\mathbf{4 a}-\mathbf{4 c}$ and final deprotection.......... 27

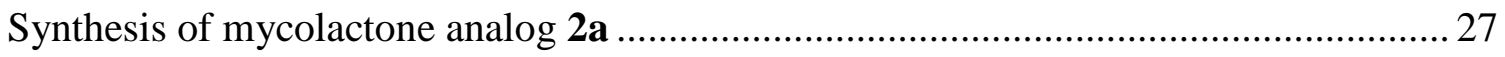

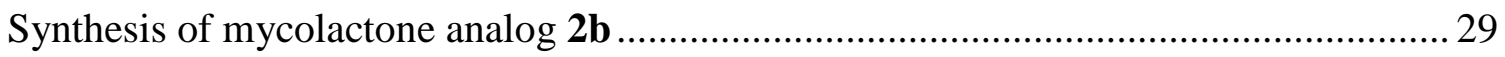

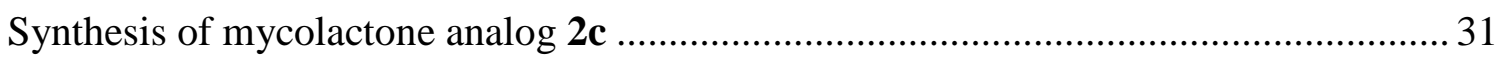

2.7 Optimization of the Stille coupling between stannane 14 and vinyl iodide 12 ............. 35

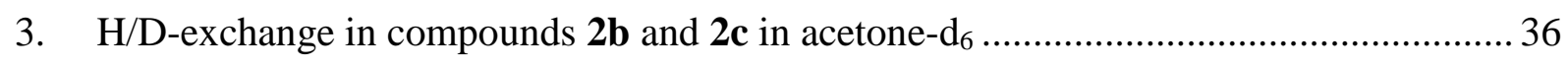

3.1 Note added during revision.............................................41

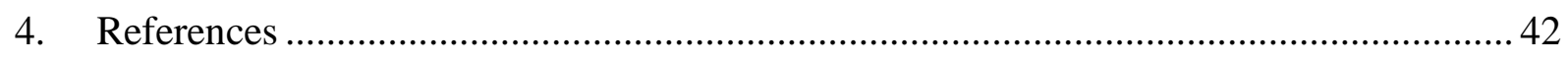

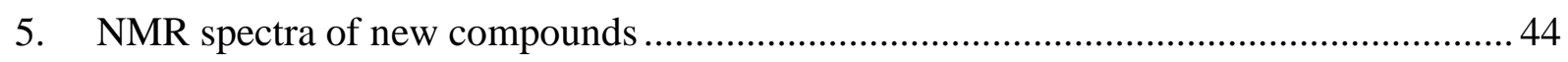




\section{Molecular modeling}

Method: The Schrödinger software package, release 2018-2 (Schrödinger, LLC, New York, NY, 2012) was used for modeling. All compounds were first minimised (PRCG, max. iteration, gradient 0.05 ). The complete $\mathrm{C} 5$-side chains were subsequently subjected to a conformational search (FF: OPLS3e, solvent: water, cut-off: extended; mixed torsional/low mode, torsion samp. method: intermediate, \#steps: 1000, 100 per rot. bond) while the macrocycle and the C11 core extension were frozen. The resulting 100-300 conformations were clustered into 33 clusters. The 10 lowest energy clusters were finally superimposed on the basis of the common macrocycle and the upper core extension.

\section{Results:}

The superimposition of the ten lowest energy clusters of mycolactones A and B (1a and $\mathbf{1 b})$ shows mycolactone A (1a) to favour folded conformations while mycolactone B (1b) preferably adopts extended conformations (Figure S1).

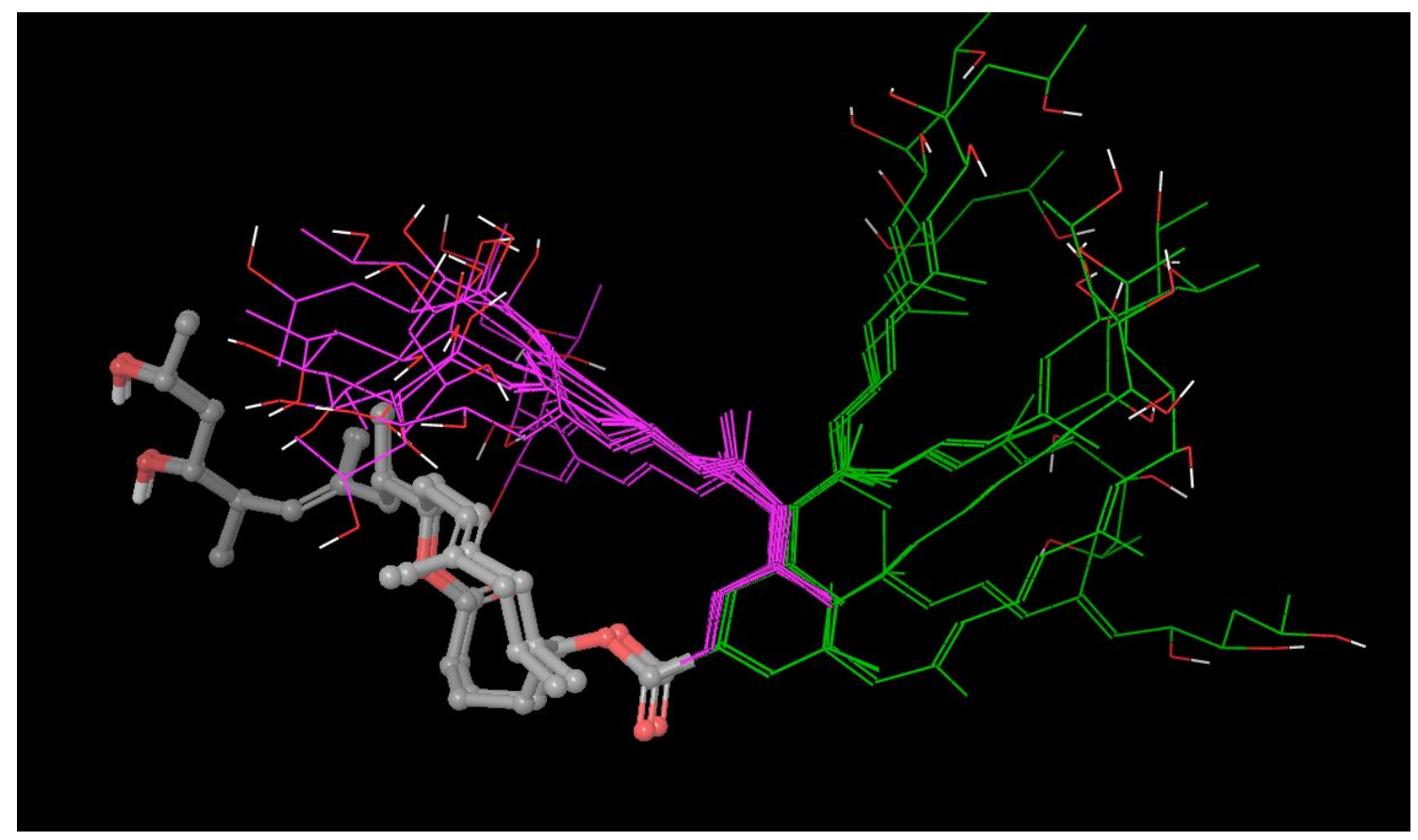

Figure S1: Superimposition of the lowest energy clusters of mycolactones A and B (1a and $\mathbf{1 b})$. The macrocycle and the upper core extension (C11-side chain) are shown in ball and stick representation, the $\mathrm{C} 5$-side chains are depicted as lines. Mycolactone A $\left(Z-\Delta^{4,5^{\prime}}\right)(\mathbf{1 a})$ : magenta. Mycolactone B $\left(E-\Delta^{4,5}\right)(\mathbf{1 b})$ : green. 
The superimposition of the ten lowest energy clusters of mycolactone B (1b) with those of the active analog $\mathbf{2 a}$ shows that $\mathbf{2 a}$ preferably adopts similar extended conformations as the natural product (Figure S2).

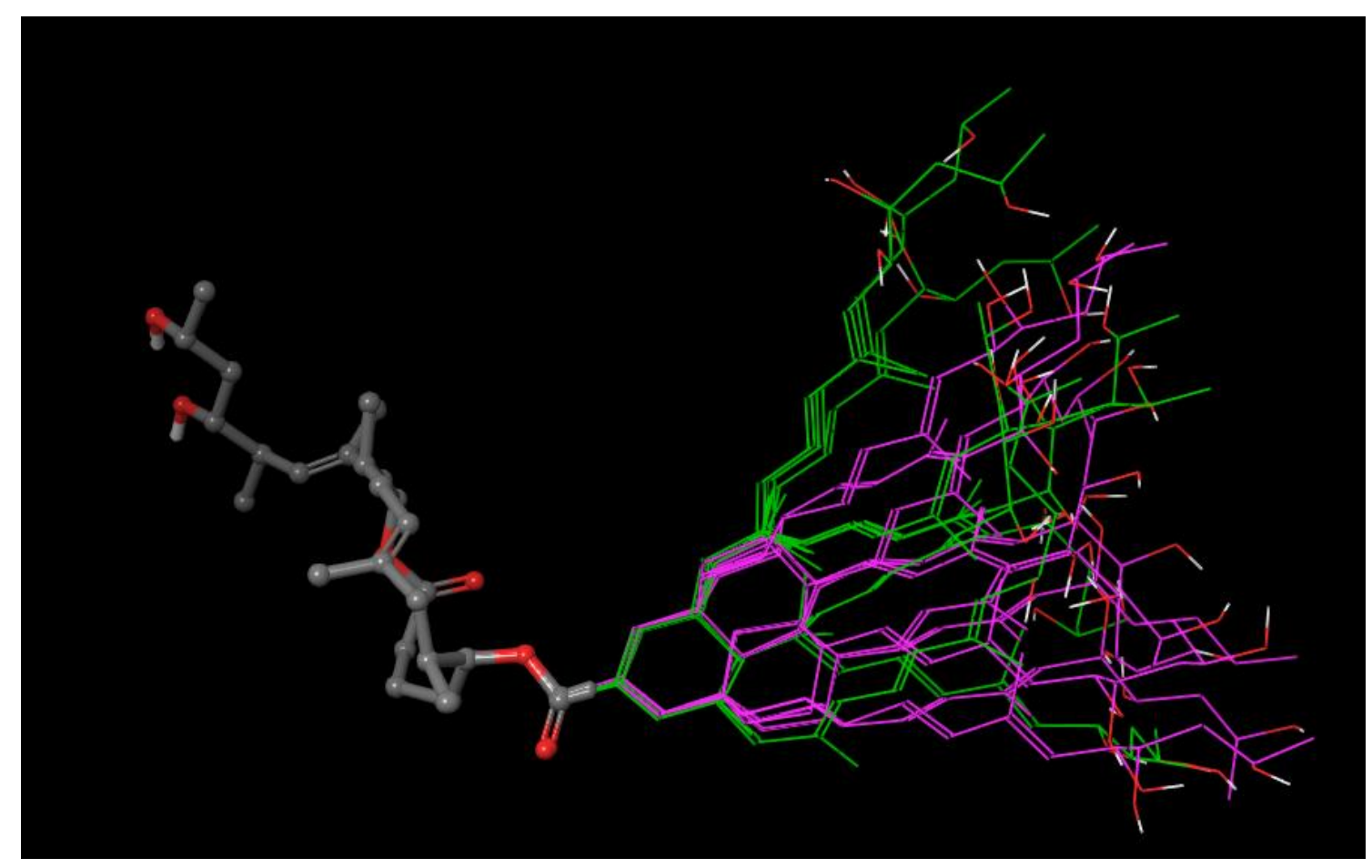

Figure S2: Superimposition of the lowest energy clusters of mycolactone B (1b) (green) and mycolactone analog 2a (magenta). The macrocycle and the upper core extension (C11-side chain) are depicted in ball and stick representation, the $\mathrm{C} 5$-side chains are shown as lines.

The low energy clusters of mycolactone analogs $\mathbf{2 b}$ and $\mathbf{2 c}$ clearly resemble the cluster of mycolactone A (1a) and are strongly dissimilar from those of either mycolactone B (1b) or $\mathbf{2 a}$ (Figure S3). 

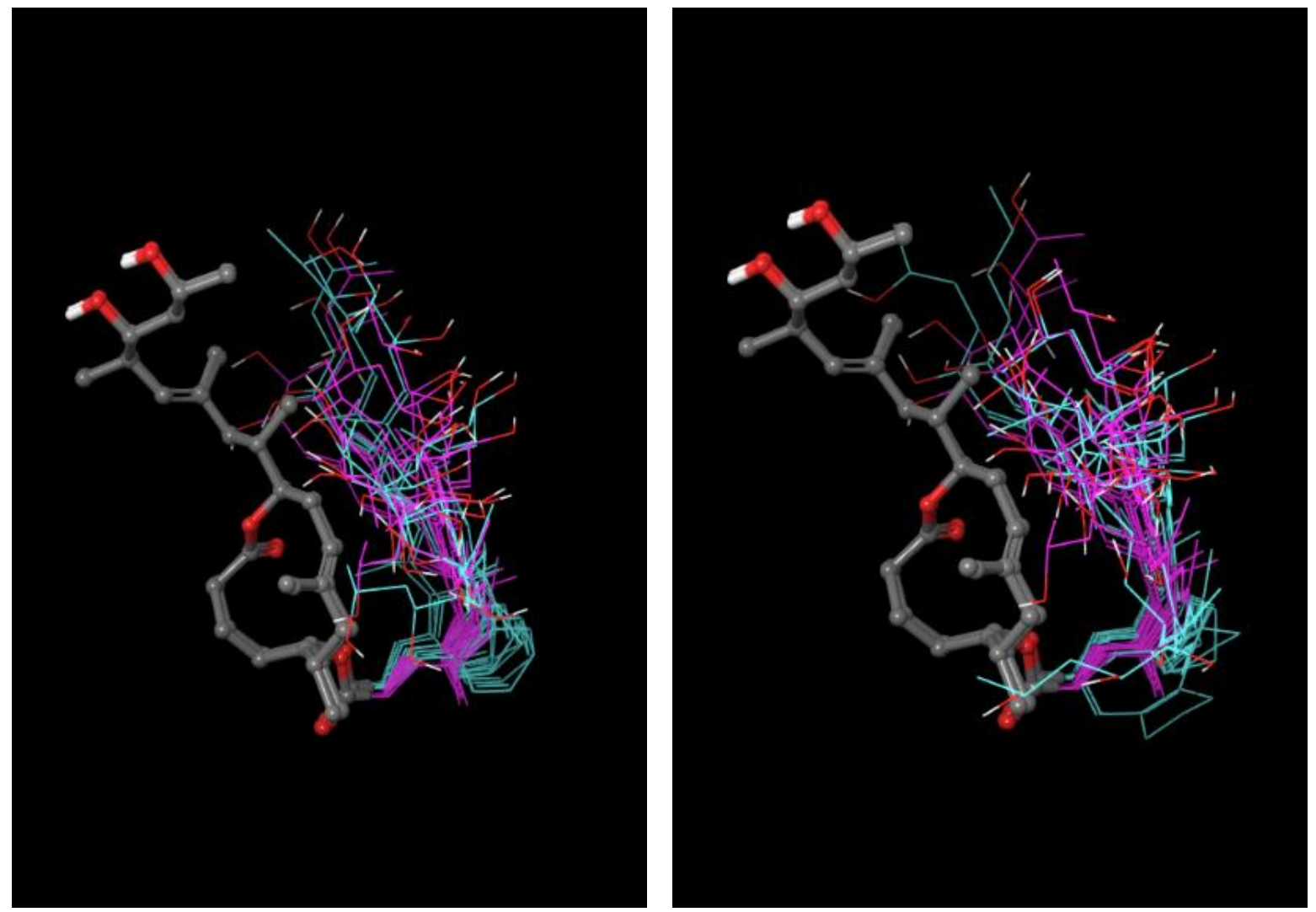

Figure S3: Superimposition of the lowest energy clusters of mycolactone A (1a) and mycolactone analogs $2 \mathbf{b}$ (left) and 2c (right). Mycolactone A (1a) is shown in magenta. Mycolactone analogs $\mathbf{2 b}$ and $\mathbf{2 c}$ are shown in cyan. The macrocycle and the upper core extension (C11-side chain) are depicted in ball and stick representation, the C5side chains are shown as lines. 


\section{Experimental Procedures}

\subsection{General Information}

All non-aqueous reactions were performed in flame-dried glassware under argon atmosphere and standard septum/syringe techniques were used. THF, $\mathrm{Et}_{2} \mathrm{O}, \mathrm{CH}_{2} \mathrm{Cl}_{2}$, and $\mathrm{Et}_{3} \mathrm{~N}$ were distilled ( $\mathrm{Et}_{3} \mathrm{~N}$ and $\mathrm{CH}_{2} \mathrm{Cl}_{2}$ from $\mathrm{CaH}_{2}$, THF and $\mathrm{Et}_{2} \mathrm{O}$ from $\mathrm{Na} /$ benzophenone) under argon atmosphere before to use. All other dry solvents $\left(\mathrm{H}_{2} \mathrm{O}<0.005 \%\right)$ were purchased over molecular sieves in septum bottles from standard suppliers (Sigma-Aldrich, Acros) and used without further purification. Solvents used for flash column chromatography (FC), thin layer chromatography (TLC) and extractions were purchased as commercial grade; EtOAc, $n$-hexane and $\mathrm{Et}_{2} \mathrm{O}$ were distilled prior to use. Generally, commercially available reagents were used without purification unless stated otherwise. Reaction mixtures were stirred magnetically and progress was monitored by TLC using Merck TLC aluminum sheets (silica gel $60 \mathrm{~F}_{254}$ ). Spots were visualized by staining with $\mathrm{Ce}_{2}\left(\mathrm{SO}_{4}\right)_{3} /$ phosphomolybdic $\quad \mathrm{acid} / \mathrm{H}_{2} \mathrm{SO}_{4} \quad$ (CPS), $\mathrm{KMnO}_{4} / \mathrm{K}_{2} \mathrm{CO}_{3} / \mathrm{NaOH}$ or, if possible, with UV light $(\lambda=254 \mathrm{~nm})$. Chromatographic purification of products by FC was performed using Silicycle SiliaFlash ${ }^{\circledR} \mathrm{P} 6$ silica gel or Fluka silica gel 60 (particle size 40-63 $\mu \mathrm{m}$ ). Melting points were obtained in open capillary tubes on a Büchi B-540 melting point apparatus and are uncorrected. ${ }^{1} \mathrm{H}$ - and ${ }^{13} \mathrm{C}-\mathrm{NMR}$ spectra were recorded in $\mathrm{CDCl}_{3}$, DMSO-d $\mathrm{d}_{6}$ or acetone- $\mathrm{d}_{6}$ on a Bruker AV-400 $400 \mathrm{MHz}$ spectrometer or on a Bruker AV-500 $500 \mathrm{MHz}$ spectrometer at room temperature. Chemical shifts $(\delta)$ are reported in ppm and are referenced to the respective solvent (chloroform $\delta 7.26 \mathrm{ppm}$ for ${ }^{1} \mathrm{H}, \delta 77.16 \mathrm{ppm}$

for ${ }^{13} \mathrm{C}$; DMSO $\delta 2.50 \mathrm{ppm}$ for ${ }^{1} \mathrm{H}, \delta 39.52 \mathrm{ppm}$ for ${ }^{13} \mathrm{C}$; acetone $\delta 2.05 \mathrm{ppm}$ for ${ }^{1} \mathrm{H}, \delta 206.26$ ppm for ${ }^{13} \mathrm{C}$ ). All ${ }^{13} \mathrm{C}$-NMR spectra were recorded with full proton decoupling. Data for NMR spectra are reported as follows: $\mathrm{s}=$ singlet, $\mathrm{d}=$ doublet, $\mathrm{t}=$ triplet, $\mathrm{q}=$ quartet, $\mathrm{m}=$ multiplet, $\mathrm{br}=$ broad signal, $J=$ coupling constant in Hz. Infrared spectra (IR) were recorded on a Jasco FT/IR-6200 instrument. Resonance frequencies are reported as wavenumbers in $\mathrm{cm}^{-1}$. Optical rotations were determined on an Anton-Paar MCP 300 polarimeter at the sodium D-line with a path length of $10 \mathrm{~mm}$ or $100 \mathrm{~mm}$, respectively, and are reported as follows: $[\alpha]_{\mathrm{D}} \mathbf{p}^{25}$ : (concentration $[\mathrm{g} / 100 \mathrm{~mL}]$, and solvent). High resolution mass spectra (HR-MS) were recorded by the ETH Zürich MS service on a Bruker maXis (ESI) or on a Micromass (Waters) AutoSpec Ultima spectrometer (EI), respectively. 


\subsection{Biological assays}

Murine L929 fibroblasts were grown in RPMI medium (Gibco) supplemented with 10\% FCS (Sigma), $2 \mathrm{mM}$ glutamine (Gibco) and incubated at $37{ }^{\circ} \mathrm{C}$ and $5 \% \mathrm{CO}_{2}$. For flow cytometry analysis 24,000 cells were seeded into 24-well plates (Falcon) and allowed to adhere o/n. Medium was then aspirated and replaced by $0.5 \mathrm{~mL}$ medium containing different concentrations of the respective mycolactone and $0.12 \% \mathrm{DMSO}$ (vol/vol). After incubation for $48 \mathrm{~h}$, cells were detached from the culture plates by repeated gentle flushing through a pipette tip without use of Trypsin-EDTA.

Harvested cells were centrifuged for $10 \mathrm{~min}$ at $1,200 x \mathrm{~g}$, resuspended in $0.3 \mathrm{~mL}$ binding buffer with $0.2 \mu \mathrm{g} / \mathrm{mL}$ Annexin V-FITC (AnnexinV kit, Calbiochem) and incubated for $30 \mathrm{~min}$ at $4{ }^{\circ} \mathrm{C}$. The cells were spun again and pellets were resuspended in $0.3 \mathrm{~mL}$ staining buffer containing $0.3 \mu \mathrm{g} / \mathrm{mL}$ propidium iodide (AnnexinV kit, Calbiochem). The cells were washed twice in $0.5 \mathrm{~mL}$ of Dulbecco PBS, spun again and pellets were resuspended in $0.3 \mathrm{~mL}$ of $1 \mathrm{x}$ binding buffer.

Cell suspensions were analysed by flow cytometry using a BD FACS Calibur Flow Cytometer (Becton Dickinson) and apoptotic $\left(\mathrm{A}^{+} / \mathrm{PI}^{-}\right)$and necrotic $\left(\mathrm{A}^{+} / \mathrm{PI}^{+}\right)$cell populations were determined using the CellQuest Pro Software (Becton Dickinson). The experiment was set up in duplicates and repeated several times; results of a representative experiment are shown. 


\subsection{Preparation of side chain acid 4a}

2.3.1 Synthesis of the western 1-phenyl- $1 H$-tetrazol-5-yl sulfone building block 7

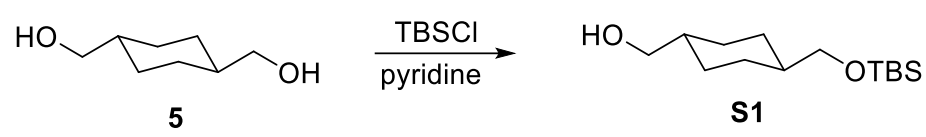

((1r,4r)-4-(((tert-Butyldimethylsilyl)oxy)methyl)cyclohexyl)methanol (S1). To a solution of trans-(cyclohexane-1,4-diyl)dimethanol $(5,9.57 \mathrm{~g}, 66.3 \mathrm{mmol})$ in $\mathrm{CH}_{2} \mathrm{Cl}_{2}(12 \mathrm{~mL})$ was added pyridine $(5.4 \mathrm{~mL}, 66.3 \mathrm{mmol})$ and a solution of $\mathrm{TBSCl}(5.00 \mathrm{~g}, 33.2 \mathrm{mmol})$ in $\mathrm{CH}_{2} \mathrm{Cl}_{2}(3 \mathrm{~mL}+$ $1 \mathrm{~mL}$ for rinsing) at $0{ }^{\circ} \mathrm{C}$ over $10 \mathrm{~min}$, during which period the initially colorless solution turned into a suspension. The suspension was stirred at room temperature for $20 \mathrm{~min}$ while it turned into a colorless solution again. Stirring was continued at room temperature for $21 \mathrm{~h}$. Water (50 $\mathrm{mL})$ and $\mathrm{Et}_{2} \mathrm{O}(100 \mathrm{~mL})$ were added and the layers were separated. The organic layer was washed with water $(50 \mathrm{~mL})$, sat. aq. $\mathrm{NH}_{4} \mathrm{Cl}$ solution $(50 \mathrm{~mL}), 10 \%$ aq. $\mathrm{CuSO}_{4}$ solution $(2 \times 10$ $\mathrm{mL})$, water $(2 \times 10 \mathrm{~mL})$ and brine $(10 \mathrm{~mL})$, dried over $\mathrm{MgSO}_{4}$ and concentrated under reduced pressure. Purification of the residue by FC ( $n$-hexane/EtOAc 9:1 $\rightarrow 6: 1$ ) gave $5.86 \mathrm{~g}$ (22.7 $\mathrm{mmol}, 68 \%$ ) of the mono-TBS protected diol $\mathbf{S} \mathbf{1}^{1}$ as a colorless oil.

${ }^{1} \mathbf{H}-\mathbf{N M R}\left(400 \mathrm{MHz}, \mathrm{CDCl}_{3}\right) \delta 3.43(\mathrm{~d}, J=6.3 \mathrm{~Hz}, 2 \mathrm{H}), 3.39(\mathrm{~d}, J=6.3 \mathrm{~Hz}, 2 \mathrm{H}), 1.90-1.73$ $(\mathrm{m}, 4 \mathrm{H}), 1.67(\mathrm{~s}, 1 \mathrm{H}), 1.50-1.34(\mathrm{~m}, 2 \mathrm{H}), 0.97-0.89(\mathrm{~m}, 4 \mathrm{H}), 0.88(\mathrm{~s}, 9 \mathrm{H}), 0.02(\mathrm{~s}, 6 \mathrm{H}) ;{ }^{13} \mathrm{C}-$ NMR $\left(101 \mathrm{MHz}, \mathrm{CDCl}_{3}\right) \delta$ 68.89, 68.87, 40.90, 40.77, 29.16, 29.12, 26.13, 18.55, -5.18.

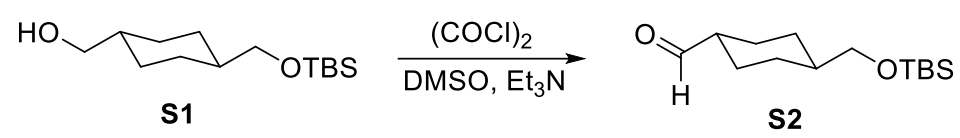

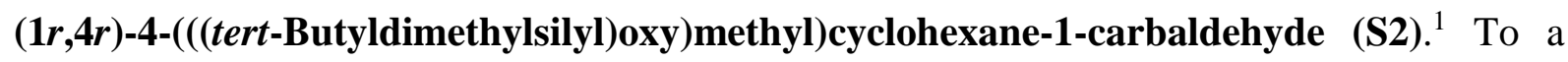
solution of oxalyl chloride $(0.65 \mathrm{~mL}, 7.74 \mathrm{mmol})$ in $\mathrm{CH}_{2} \mathrm{Cl}_{2}(20 \mathrm{~mL})$ cooled to $-78{ }^{\circ} \mathrm{C}$ was added DMSO (1.10 mL, $15.5 \mathrm{mmol})$ slowly and dropwise. After stirring for $10 \mathrm{~min}$ at $-78{ }^{\circ} \mathrm{C}$ the alcohol $\mathbf{S 1}(1.00 \mathrm{~g}, 3.87 \mathrm{mmol})$ in $\mathrm{CH}_{2} \mathrm{Cl}_{2}(10 \mathrm{~mL}+5 \mathrm{~mL}$ for rinsing $)$ was added via dropping funnel. After stirring for another $10 \mathrm{~min}$ at $-78{ }^{\circ} \mathrm{C}, \mathrm{Et}_{3} \mathrm{~N}(2.69 \mathrm{~mL}, 19.3 \mathrm{mmol})$ was added dropwise and the solution was allowed to warm to room temperature. Water $(50 \mathrm{~mL})$ and $\mathrm{Et}_{2} \mathrm{O}(100 \mathrm{~mL})$ were added and the layers were separated. The organic layer was washed with water $(50 \mathrm{~mL})$. The combined aqueous layers were re-extracted with $\mathrm{Et}_{2} \mathrm{O}(50 \mathrm{~mL})$. The combined organic layers were washed with $1 \mathrm{M}$ aq. $\mathrm{HCl}(40 \mathrm{~mL})$, water $(50 \mathrm{~mL})$, sat. aq. $\mathrm{NaHCO}_{3}$ solution $(50 \mathrm{~mL})$ and brine $(20 \mathrm{~mL})$, dried over $\mathrm{MgSO}_{4}$ and concentrated under reduced pressure. Purification of the residue by FC ( $n$-hexane/Et $2 \mathrm{O} 20: 1 \rightarrow 9: 1$ ) gave $900 \mathrm{mg}$ (3.51 mmol, 91\%) of aldehyde $\mathbf{S 2}^{1}$ as a colorless oil. 
${ }^{1} \mathbf{H}-\mathbf{N M R}\left(400 \mathrm{MHz}, \mathrm{CDCl}_{3}\right) \delta 9.61(\mathrm{~d}, J=1.6 \mathrm{~Hz}, 1 \mathrm{H}), 3.42(\mathrm{~d}, J=6.2 \mathrm{~Hz}, 2 \mathrm{H}), 2.16(\mathrm{ttd}, J=$ 12.2, 3.6, $1.7 \mathrm{~Hz}, 1 \mathrm{H}), 2.05-1.94(\mathrm{~m}, 2 \mathrm{H}), 1.93-1.81$ (m, 2H), $1.50-1.37$ (m, 1H), 1.27 (qd, $J=13.1,3.6 \mathrm{~Hz}, 2 \mathrm{H}), 0.98(\mathrm{qd}, J=13.1,3.4 \mathrm{~Hz}, 2 \mathrm{H}), 0.89$ (s, 9H), 0.03 (s, 6H); ${ }^{13} \mathbf{C}-\mathbf{N M R}$ $\left(101 \mathrm{MHz}, \mathrm{CDCl}_{3}\right) \delta 204.69,68.27,50.59,39.95,28.30,25.95,25.55,18.36,-5.37$.
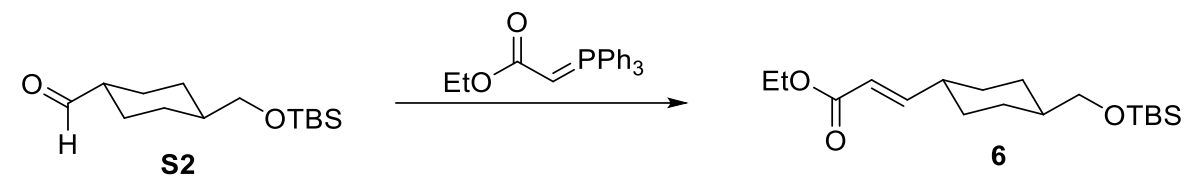

Ethyl (E)-3-((1r,4r)-4-(((tert-butyldimethylsilyl)oxy)methyl)cyclohexyl)acrylate (6). To a solution of aldehyde $\mathbf{S 2}(900 \mathrm{mg}, 3.51 \mathrm{mmol})$ in $\mathrm{CH}_{2} \mathrm{Cl}_{2}(25 \mathrm{~mL})$ was added (triphenylphosphoranylidene)acetic acid ethyl ester (1.41 g, $4.04 \mathrm{mmol})$. The colorless solution was stirred at room temperature for $20 \mathrm{~h}$. After $16 \mathrm{~h}$, additional phosphorane $(122 \mathrm{mg}$, $0.35 \mathrm{mmol}$ ) was added. Silica gel and additional $\mathrm{CH}_{2} \mathrm{Cl}_{2}$ were added and the volatiles were evaporated under reduced pressure. Purification by FC ( $n$-hexane/EtOAc 20:1) gave $1.09 \mathrm{~g}$ (3.34 mmol, 95\%) of ester $\mathbf{6}$ as a mixture of isomers (trans/cis 19:1) as a colorless oil. $\mathbf{R}_{\mathbf{f}}(n$ hexane/EtOAc 9:1) = 0.52; ${ }^{1} \mathrm{H}-\mathrm{NMR}\left(400 \mathrm{MHz}, \mathrm{CDCl}_{3}\right): \delta 6.91(\mathrm{dd}, J=15.8,6.8 \mathrm{~Hz}, 1 \mathrm{H})$, $5.76(\mathrm{dd}, J=15.8,1.4 \mathrm{~Hz}, 1 \mathrm{H}), 4.17(\mathrm{q}, J=7.1 \mathrm{~Hz}, 2 \mathrm{H}),, 3.40(\mathrm{~d}, J=6.3 \mathrm{~Hz}, 2 \mathrm{H}), 2.16-1.96$ (m, 1H), $1.91-1.72(\mathrm{~m}, 4 \mathrm{H}), 1.49-1.35(\mathrm{~m}, 1 \mathrm{H}), 1.28(\mathrm{t}, J=7.1 \mathrm{~Hz}, 3 \mathrm{H}), 1.22-1.07(\mathrm{~m}, 2 \mathrm{H})$, $1.09-0.91(\mathrm{~m}, 2 \mathrm{H}), 0.89$ (s, 9H), 0.03 (s, 6H); ${ }^{13}$ C-NMR (100 MHz, $\left.\mathrm{CDCl}_{3}\right): 167.25,154.18$, $119.15,68.67,60.29,40.86,40.07,31.31,29.08,26.11,18.53,14.42,-5.21$; IR (neat): $\widetilde{v}=$ 2961 2926, 2855, 1721, 1651, 1471, 1449, 1389, 1365, 1299, 1255, 1216, 1176, 1150, 1107 , 1094, 1052, 1006, 985, 941, 834, 814, 774, 666; HR-MS (ESI) calc. 327.2350 for $\mathrm{C}_{18} \mathrm{H}_{35} \mathrm{O}_{3} \mathrm{Si}^{+}$ $[\mathrm{M}+\mathrm{H}]^{+}$, found 327.2359 .
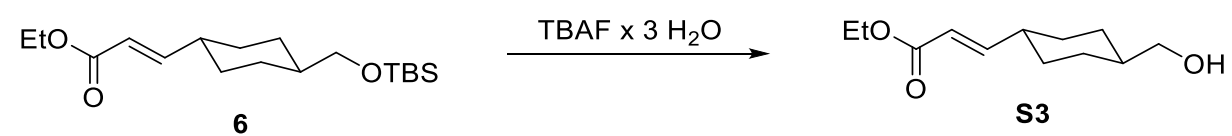

Ethyl (E)-3-((1r,4r)-4-(hydroxymethyl)cyclohexyl)acrylate (S3). To a solution of ester 6 $(1.09 \mathrm{~g}, 3.34 \mathrm{mmol})$ in THF $(20 \mathrm{~mL})$ was added TBAF. $3 \mathrm{H}_{2} \mathrm{O}(1.37 \mathrm{~g}, 4.34 \mathrm{mmol})$ at room temperature. The yellow solution was stirred at room temperature for $2.5 \mathrm{~h}$ before being concentrated under reduced pressure. Purification by FC ( $n$-hexane/EtOAc 4:1 $\rightarrow$ 1:1, 0.5\% $\mathrm{MeOH})$ gave $700 \mathrm{mg}$ (3.30 mmol, 99\%) of alcohol $\mathbf{S 3}$ as a colorless oil. $\mathbf{R}_{\mathbf{f}}$ ( $n$-hexane/EtOAc 2:1) $=0.30 ;{ }^{1} \mathbf{H}-\mathbf{N M R}\left(400 \mathrm{MHz}, \mathrm{CDCl}_{3}\right): \delta 6.91(\mathrm{dd}, J=15.8,6.8 \mathrm{~Hz}, 1 \mathrm{H}), 5.77(\mathrm{dd}, J=15.8$, $1.4 \mathrm{~Hz}, 1 \mathrm{H}), 4.18$ (q, $J=7.1 \mathrm{~Hz}, 2 \mathrm{H}), 3.47$ (d, $J=6.3 \mathrm{~Hz}, 2 \mathrm{H}), 2.20-1.99(\mathrm{~m}, 1 \mathrm{H}), 1.89-1.80$ 
(m, 4H), $1.63(\mathrm{~s}, 1 \mathrm{H}), 1.52-1.40(\mathrm{~m}, 1 \mathrm{H}), 1.28(\mathrm{t}, J=7.1 \mathrm{~Hz}, 3 \mathrm{H}), 1.18$ (ddd, $J=15.7,13.3$, $3.5 \mathrm{~Hz}, 2 \mathrm{H}), 1.02$ (ddd, $J=16.9,13.1,3.4 \mathrm{~Hz}, 2 \mathrm{H}) ;{ }^{13} \mathrm{C}-\mathbf{N M R}\left(100 \mathrm{MHz}, \mathrm{CDCl}_{3}\right): \delta$ 167.05, 153.73, 119.13, 68.39, 60.18, 40.53, 39.91, 31.03, 28.78, 14.26; IR (film, EtOAc): $\widetilde{v}=3422$, 2920, 2852, 1716, 1700, 1649, 1448, 1392, 1368, 1299, 1273, 1240, 1216, 1177, 1147, 1133, 1114, 1096, 1065, 1035, 984, 949, 863; HR-MS (ESI) calc. 235.1305 for $\mathrm{C}_{12} \mathrm{H}_{20} \mathrm{NaO}_{3}{ }^{+}$ $[\mathrm{M}+\mathrm{Na}]^{+}$, found 235.1309 .
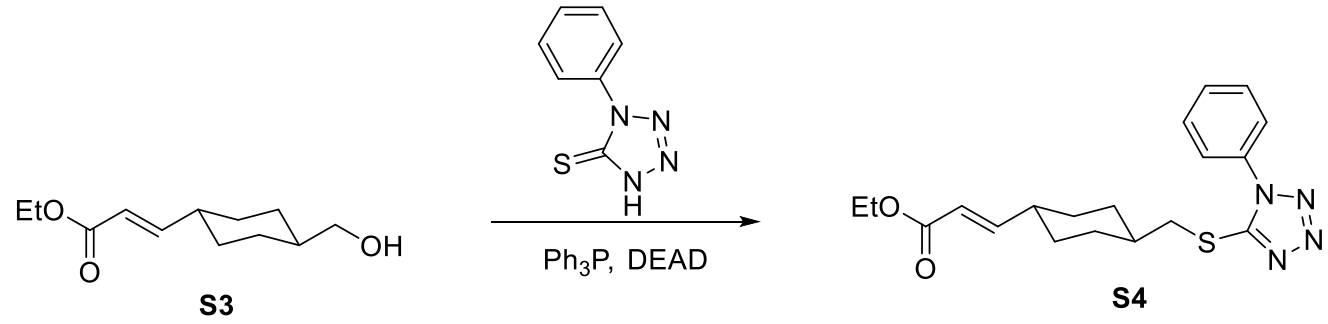

Ethyl $(E)-3-((1 r, 4 r)-4-(((1-p h e n y l-1 H$-tetrazol-5-yl)thio)methyl)cyclohexyl)acrylate (S4). Alcohol S3 (100 mg, $471 \mu \mathrm{mol})$, triphenylphosphine (185 mg, $70.5 \mu \mathrm{mol})$ and 1-phenyl-2tetrazoline-5-thione (168 mg, $943 \mu \mathrm{mol})$ were dissolved in THF $(4.0 \mathrm{~mL})$ at room temperature. After cooling the solution to $0{ }^{\circ} \mathrm{C}$, diisopropyl azodicarboxylate (DIAD; $162 \mu \mathrm{L}, 824 \mu \mathrm{mol}$ ) was added dropwise. The resulting orange solution was allowed to warm to room temperature and stirred for 100 min over which period it turned colorless. The reaction was quenched by adding sat. aq. $\mathrm{NaHCO}_{3}$ solution $(10 \mathrm{~mL})$, water $(10 \mathrm{~mL})$ and $\mathrm{Et}_{2} \mathrm{O}(20 \mathrm{~mL})$. The layers were separated and the aqueous layer was extracted with $\mathrm{Et}_{2} \mathrm{O}(2 \times 20 \mathrm{~mL})$. The combined organic layers were dried over $\mathrm{MgSO}_{4}$ and concentrated under reduced pressure. Purification by $\mathrm{FC}$ ( $n$ hexane/EtOAc 9:1) gave $157 \mathrm{mg}(421 \mu \mathrm{mol}, 89 \%)$ of sulfide S4 as a colorless oil which solidified in the freezer overnight. $\mathbf{R}_{\mathbf{f}}(n$-hexane/EtOAc $4: 1)=0.29 ; \mathbf{m} . \mathbf{p} .=68{ }^{\circ} \mathrm{C} ;{ }^{1} \mathbf{H}-\mathbf{N M R}$ $\left(400 \mathrm{MHz}, \mathrm{CDCl}_{3}\right): \delta 7.64-7.45(\mathrm{~m}, 5 \mathrm{H}), 6.87(\mathrm{dd}, J=15.8,6.8 \mathrm{~Hz}, 1 \mathrm{H}), 5.75(\mathrm{dd}, J=15.8$, $1.4 \mathrm{~Hz}, 1 \mathrm{H}), 4.17$ (q, $J=7.1 \mathrm{~Hz}, 2 \mathrm{H}), 3.32$ (d, $J=6.9 \mathrm{~Hz}, 2 \mathrm{H}), 2.24-2.03$ (m, 1H), $2.03-1.92$ $(\mathrm{m}, 2 \mathrm{H}), 1.89-1.80(\mathrm{~m}, 2 \mathrm{H}), 1.80-1.69(\mathrm{~m}, 1 \mathrm{H}), 1.26(\mathrm{t}, J=7.1 \mathrm{~Hz}, 3 \mathrm{H}), 1.25-1.01(\mathrm{~m}$, 5H); ${ }^{13}$ C-NMR $\left(100 \mathrm{MHz}, \mathrm{CDCl}_{3}\right): \delta 166.88,153.08,130.06,129.75,123.81,119.38,60.19$, 40.05, 39.87, 36.85, 31.57, 31.01, 14.22; IR (film, EtOAc): $\widetilde{v}=2924,2851,2369,2363,2340$, 1713,1652 , 1597, 1500, 1448, 1411, 1387, 1367, 1300, 1275, 1237, 1213, 1185, 1130, 1090, 1074, 1041, 1015, 984, 946, 761, 694, 686; HR-MS (ESI) calc. 373.1693 for $\mathrm{C}_{19} \mathrm{H}_{25} \mathrm{~N}_{4} \mathrm{O}_{2} \mathrm{~S}^{+}$ $[\mathrm{M}+\mathrm{H}]^{+}$, found 373.1695 . 


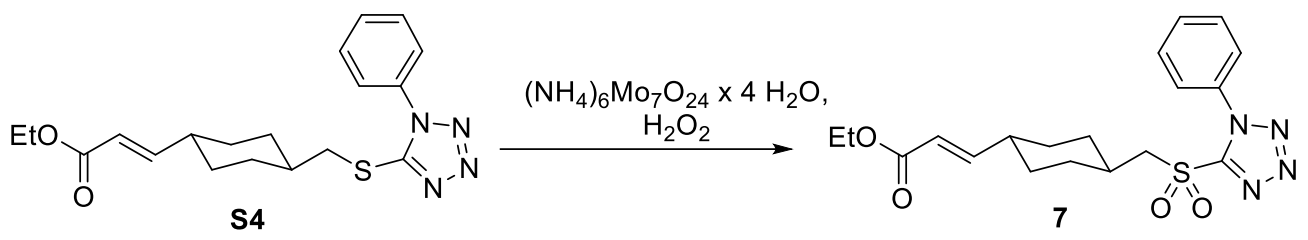

Ethyl (E)-3-((1r,4r)-4-(((1-phenyl-1H-tetrazol-5-yl)sulfonyl)methyl)cyclohexyl)acrylate (7). To sulfide $\mathbf{S 4}(154 \mathrm{mg}, 413 \mu \mathrm{mol})$ in EtOH/THF/ $\mathrm{H}_{2} \mathrm{O}(5: 4: 1,4 \mathrm{~mL})$ were added ammonium molybdate tetrahydrate $(102 \mathrm{mg}, 82.7 \mu \mathrm{mol})$ and $\mathrm{H}_{2} \mathrm{O}_{2}\left(50 \%\right.$ in $\left.\mathrm{H}_{2} \mathrm{O}, 281 \mu \mathrm{L}, 4.13 \mathrm{mmol}\right)$ at room temperature. The white suspension was stirred at room temperature for $6 \mathrm{~h}$ over which period it turned yellow. The reaction was quenched by adding $10 \%$ aq. $\mathrm{Na}_{2} \mathrm{~S}_{2} \mathrm{O}_{3}$ solution (25 mL). The EtOH was partly removed under reduced pressure, $\mathrm{Et}_{2} \mathrm{O}(50 \mathrm{~mL})$ was added and the layers were separated. The aqueous layer was extracted with $\mathrm{Et}_{2} \mathrm{O}(2 \times 20 \mathrm{~mL})$ and the combined organic layers were washed with water $(5 \mathrm{~mL})$ and brine, dried over $\mathrm{MgSO}_{4}$ and concentrated under reduced pressure. Purification by FC ( $n$-hexane/EtOAc 12:1 $\rightarrow$ 9:1 $\rightarrow$ 4:1 $\rightarrow 1: 1)$ yielded $159 \mathrm{mg}$ (393 $\mu \mathrm{mol}, 95 \%)$ of sulfone 7 as a colorless oil. $\mathbf{R}_{\mathbf{f}}(n$-hexane/EtOAc 4:1) = 0.19; ${ }^{1} \mathbf{H}-\mathbf{N M R}\left(400 \mathrm{MHz}, \mathrm{CDCl}_{3}\right): \delta 7.72-7.56(\mathrm{~m}, 5 \mathrm{H}), 6.85(\mathrm{dd}, J=15.8,6.8 \mathrm{~Hz}$, 1H), $5.76(\mathrm{dd}, J=15.8,1.4 \mathrm{~Hz}, 1 \mathrm{H}), 4.18(\mathrm{q}, J=7.1 \mathrm{~Hz}, 2 \mathrm{H}), 3.70(\mathrm{~d}, J=6.2 \mathrm{~Hz}, 2 \mathrm{H}), 2.21-$ $2.03(\mathrm{~m}, 4 \mathrm{H}), 1.92-1.79(\mathrm{~m}, 2 \mathrm{H}), 1.32-1.15(\mathrm{~m}, 7 \mathrm{H}) ;{ }^{13} \mathbf{C}-\mathbf{N M R}\left(100 \mathrm{MHz}, \mathrm{CDCl}_{3}\right): \delta 166.80$, $152.49,131.47,129.69,125.06,119.64,61.80,60.26,39.50,32.16,32.09,30.88,14.23$; IR (film, EtOAc): $\widetilde{v}=2928,2855,1712,1650,1498,1449,1341,1303,1277,1234,1215,1184$, 1152, 1097, 1045, 1015, 985, 859, 764, 690, 632, 613, 548, 519; HR-MS (ESI) calc. 405.1591 for $\mathrm{C}_{19} \mathrm{H}_{25} \mathrm{~N}_{4} \mathrm{O}_{4} \mathrm{~S}^{+}[\mathrm{M}+\mathrm{H}]^{+}$, found 405.1588 .

\subsubsection{Assembly of sulfone 7 and aldehyde 8 by Julia-Kocienski olefination and ester cleavage}
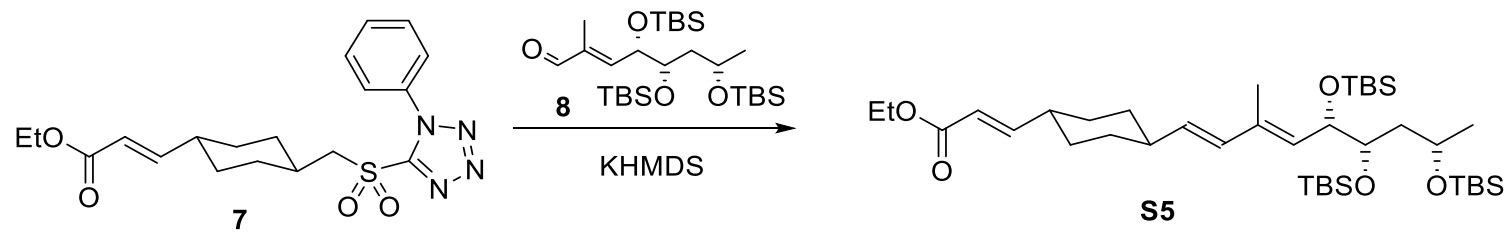

Ethyl $(E)-3-((1 S, 4 r)-4-((1 E, 3 E, 5 S, 6 S, 8 S)-5,6,8$-tris((tert-butyldimethylsilyl)oxy)-3-methylnona-1,3-dien-1-yl)cyclohexyl)acrylate (S5). To a solution of sulfone 7 (74.0 mg, $183 \mu \mathrm{mol})$ in THF $(1.5 \mathrm{~mL})$ was added KHMDS $(0.5 \mathrm{M}$ in toluene, $320 \mu \mathrm{L}, 160 \mu \mathrm{mol})$ at $-78{ }^{\circ} \mathrm{C}$. The brown solution was stirred at $-78^{\circ} \mathrm{C}$ for $5 \mathrm{~min}$ before a solution of aldehyde $\mathbf{8}^{2}$ (48.6 $\mathrm{mg}, 91.5$ $\mu \mathrm{mol})$ in THF (3.0 mL) was added over a period of $90 \mathrm{~min}$. The yellow solution then was stirred at $-78{ }^{\circ} \mathrm{C}$ for $20 \mathrm{~min}$. Sat. aq. $\mathrm{NH}_{4} \mathrm{Cl}$ solution $(10 \mathrm{~mL})$ and EtOAc $(20 \mathrm{~mL})$ were added before 
the solution was warmed to room temperature. The layers were separated and the aqueous layer was extracted with EtOAc $(2 \times 20 \mathrm{~mL})$. The combined organic layers were washed with brine, dried over $\mathrm{MgSO}_{4}$ and concentrated under reduced pressure after addition of silica. Repeated purification by FC ( $n$-hexane/EtOAc 100:1 $\rightarrow$ 20:1 $\rightarrow$ 4:1; then $n$-hexane/EtOAc 100:1) gave $40 \mathrm{mg}(56 \mu \mathrm{mol}, 62 \%)$ of the fully protected side chain $\mathbf{S 5}$ (all-trans only) as a colorless oil. Rf $\left(n\right.$-hexane/EtOAc 4:1) $=0.77 ;[\alpha] \mathbf{D}^{25}=-18.5^{\circ}\left(\mathrm{c}=0.77, \mathrm{CHCl}_{3}\right) ;{ }^{1} \mathbf{H}-\mathbf{N M R}\left(400 \mathrm{MHz}, \mathrm{CDCl}_{3}\right)$ : $\delta 6.92(\mathrm{dd}, J=15.8,6.8 \mathrm{~Hz}, 1 \mathrm{H}), 6.03(\mathrm{~d}, J=15.8 \mathrm{~Hz}, 1 \mathrm{H}), 5.78(\mathrm{dd}, J=15.8,1.4 \mathrm{~Hz}, 1 \mathrm{H})$, $5.52(\mathrm{dd}, J=15.7,6.9 \mathrm{~Hz}, 1 \mathrm{H}), 5.37(\mathrm{~d}, J=9.1 \mathrm{~Hz}, 1 \mathrm{H}), 4.39$ (dd, $J=9.1,3.7 \mathrm{~Hz}, 1 \mathrm{H}), 4.18$ $(\mathrm{q}, J=7.1 \mathrm{~Hz}, 2 \mathrm{H}), 3.96-3.85(\mathrm{~m}, 1 \mathrm{H}), 3.67-3.56(\mathrm{~m}, 1 \mathrm{H}), 2.14-1.93(\mathrm{~m}, 2 \mathrm{H}), 1.89-1.71$ $(\mathrm{m}, 8 \mathrm{H}), 1.65-1.55(\mathrm{~m}, 1 \mathrm{H}), 1.36-1.10(\mathrm{~m}, 10 \mathrm{H}), 0.88(\mathrm{~s}, 9 \mathrm{H}), 0.87(\mathrm{~s}, 9 \mathrm{H}), 0.86(\mathrm{~s}, 9 \mathrm{H})$, $0.05(\mathrm{~s}, 3 \mathrm{H}), 0.05(\mathrm{~s}, 3 \mathrm{H}), 0.04(\mathrm{~s}, 3 \mathrm{H}), 0.03(\mathrm{~s}, 3 \mathrm{H}), 0.01(\mathrm{~s}, 3 \mathrm{H}),-0.04(\mathrm{~s}, 3 \mathrm{H}) ;{ }^{13} \mathrm{C}-\mathbf{N M R}(100$ $\left.\mathrm{MHz}, \mathrm{CDCl}_{3}\right): \delta 167.23,154.09,134.28,134.17,132.99,130.57,119.24,73.82,71.53,66.29$, 60.32, 42.94, 40.57, 40.29, 32.53, 32.52, 31.50, 26.10, 26.06, 26.01, 23.69, 18.31, 18.24, 18.15, 14.44, 13.63, -3.97, -4.03, -4.14, -4.35, -4.42, -4.52; IR (film, EtOAc): $\widetilde{v}=2954,2928,2856$, 1724, 1652, 1472, 1448, 1362, 1298, 1254, 1216, 1175, 1143, 1094, 1066, 1005, 985, 963, 938, 918, 896, 834, 809, 774, 665; HR-MS (ESI) calc. 731.4893 for $\mathrm{C}_{39} \mathrm{H}_{76} \mathrm{NaO}_{5} \mathrm{Si}_{3}{ }^{+}[\mathrm{M}+\mathrm{Na}]^{+}$, found 731.4880 .
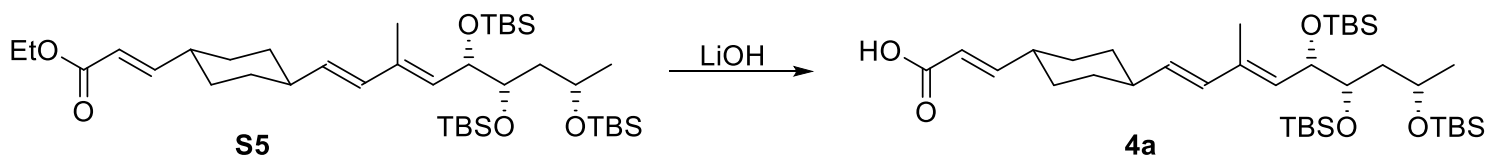

\section{(E)-3-((1S,4r)-4-((1E,3E,5S,6S,8S)-5,6,8-tris((tert-Butyldimethylsilyl)oxy)-3-methylnona-}

1,3-dien-1-yl)cyclohexyl)acrylic acid (4a). To a solution of ester S5 (47.0 mg, $66.3 \mu \mathrm{mol})$ in $\mathrm{THF} / \mathrm{MeOH} / \mathrm{H}_{2} \mathrm{O}(4: 1: 1,3 \mathrm{~mL})$ was added $\mathrm{LiOH} \cdot \mathrm{H}_{2} \mathrm{O}(27.8 \mathrm{mg}, 663 \mu \mathrm{mol})$ at room temperature. The slightly yellow solution was stirred at room temperature for $17 \mathrm{~h}$. The reaction was quenched by adding sat. aq. $\mathrm{NH}_{4} \mathrm{Cl}$ solution $(10 \mathrm{~mL})$ and EtOAc $(15 \mathrm{~mL})$, the layers were separated and the aqueous layer was extracted with EtOAc $(2 \times 15 \mathrm{~mL})$. The combined organic layers were washed with brine, dried over $\mathrm{MgSO}_{4}$ and concentrated under reduced pressure. Purification by FC ( $n$-hexane/EtOAc 20:1 $\rightarrow n$-hexane/EtOAc 9:1, 0.25\% AcOH) yielded $42 \mathrm{mg}(61.6 \mu \mathrm{mol}, 93 \%)$ of acid $\mathbf{4 a}$ as a colorless oil. $\mathbf{R} \mathbf{f}(n$-hexane/EtOAc/AcOH 80:20:1) $=$ $0.30 ;[\alpha]_{\mathbf{D}^{25}}=-15.4^{\circ}\left(\mathrm{c}=0.42, \mathrm{CHCl}_{3}\right) ;{ }^{1} \mathbf{H}-\mathbf{N M R}\left(400 \mathrm{MHz}, \mathrm{CDCl}_{3}\right): \delta 7.04(\mathrm{dd}, J=15.7,6.9$ $\mathrm{Hz}, 1 \mathrm{H}), 6.03(\mathrm{~d}, J=15.8 \mathrm{~Hz}, 1 \mathrm{H}), 5.79(\mathrm{dd}, J=15.8,1.3 \mathrm{~Hz}, 1 \mathrm{H}), 5.52(\mathrm{dd}, J=15.8,6.9 \mathrm{~Hz}$, $1 \mathrm{H}), 5.38(\mathrm{~d}, J=9.1 \mathrm{~Hz}, 1 \mathrm{H}), 4.39(\mathrm{dd}, J=9.1,3.7 \mathrm{~Hz}, 1 \mathrm{H}), 3.99-3.84(\mathrm{~m}, 1 \mathrm{H}), 3.68-3.55$ (m, 1H), $2.20-2.07(\mathrm{~m}, 1 \mathrm{H}), 2.06-1.93(\mathrm{~m}, 1 \mathrm{H}), 1.91-1.70(\mathrm{~m}, 8 \mathrm{H}), 1.61(\mathrm{ddd}, J=13.3$, 
7.8, 5.4 Hz, 1H), $1.34-1.08(\mathrm{~m}, 7 \mathrm{H}), 0.88(\mathrm{~s}, 9 \mathrm{H}), 0.87(\mathrm{~s}, 9 \mathrm{H}), 0.86(\mathrm{~s}, 9 \mathrm{H}), 0.05(\mathrm{~s}, 3 \mathrm{H}), 0.05$ (s, 3H), $0.04(\mathrm{~s}, 3 \mathrm{H}), 0.03(\mathrm{~s}, 3 \mathrm{H}), 0.01(\mathrm{~s}, 3 \mathrm{H}),-0.04(\mathrm{~s}, 3 \mathrm{H}) ;{ }^{13} \mathbf{C}-\mathbf{N M R}\left(100 \mathrm{MHz}, \mathrm{CDCl}_{3}\right): \delta$ $171.63,156.90,134.26,134.07,133.05,130.62,118.44,73.82,71.53,66.30,42.94,40.52$, $40.42,32.47,32.45,31.35,26.10,26.07,26.01,23.69,18.31,18.24,18.16,13.63,-3.97,-4.02$, $-4.14,-4.35,-4.42,-4.52$; IR (film, EtOAc): $\widetilde{v}=2955,2928,2895,2856,2357,1698,1254$, 1093, 1067, 835, 774; HR-MS (ESI) calc. 703.4580 for $\mathrm{C}_{37} \mathrm{H}_{72} \mathrm{NaO}_{5} \mathrm{Si}_{3}{ }^{+}[\mathrm{M}+\mathrm{Na}]^{+}$, found 703.4580 .

\subsection{Preparation of the side chain acid $4 b$}

\subsubsection{Synthesis of the western vinyl iodide building block $\mathbf{1 8}$}<smiles>COC(=O)c1ccccc1I</smiles>
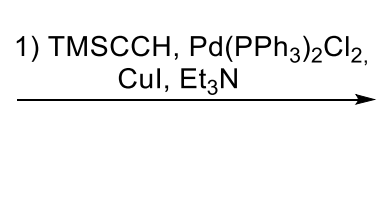

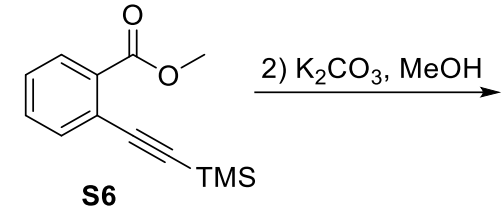

S6

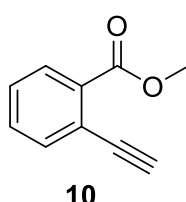

10

\section{Methyl 2-ethynylbenzoate (10) ${ }^{3}$}

Step 1) To $\mathrm{Pd}\left(\mathrm{PPh}_{3}\right)_{2} \mathrm{Cl}_{2}(268 \mathrm{mg}, 382 \mu \mathrm{mol})$ and $\mathrm{CuI}(145 \mathrm{mg}, 763 \mu \mathrm{mol})$ in THF $(75 \mathrm{~mL})$ was added methyl 2-iodobenzoate $(9,5.00 \mathrm{~g}, 19.1 \mathrm{mmol})$ via cannula followed by $\mathrm{Et}_{3} \mathrm{~N}(7.98 \mathrm{~mL}$, $57.2 \mathrm{mmol})$ and TMS-acetylene $(5.39 \mathrm{~mL}, 38.2 \mathrm{mmol})$. The suspension turned black and was stirred at ambient temperature for $1 \mathrm{~h}$. Sat. aq. $\mathrm{NH}_{4} \mathrm{Cl}$ solution $(100 \mathrm{~mL})$ and $n$-hexane $(100 \mathrm{~mL})$ were added and the aqueous layer was extracted twice with $n$-hexane/EtOAc 5:1 (150 mL). The combined organic layers were dried over $\mathrm{MgSO}_{4}$ and the solvent was removed under reduced pressure. The TMS-protected intermediate $\mathbf{S 6}^{4}$ (4.35 g, $18.7 \mathrm{mmol}, 98 \%$, crude), which was obtained as a brown oil, was immediately used in the next step without further characterization or purification.

Step 2) To a solution of crude protected alkyne S6 (4.35 g, $18.7 \mathrm{mmol})$ in $\mathrm{MeOH}$ (200 mL) was added $\mathrm{K}_{2} \mathrm{CO}_{3}(7.76 \mathrm{~g}, 56.2 \mathrm{mmol})$ and the mixture was stirred at room temperature for $14 \mathrm{~h}$. Water $(200 \mathrm{~mL})$ was added and the mixture was extracted with ether $(2 \times 150 \mathrm{~mL})$. The aq. layer was concentrated to a volume of $200 \mathrm{~mL}$ and re-extracted with ether $(2 \times 100 \mathrm{~mL})$. The combined organic layers were washed with brine, dried over $\mathrm{MgSO}_{4}$ and concentrated under reduced pressure. Purification by column chromatography (n-hexane/EtOAc 20:1) afforded $2.74 \mathrm{~g}$ (17.1 mmol, $89 \%$ over two steps) of the terminal alkyne $\mathbf{1 0}^{\mathbf{3}, 4}$ as a yellow oil. 
$\mathbf{R}_{\mathbf{f}}\left(n\right.$-hexane/EtOAc 20:1) $=0.21 ;{ }^{1} \mathbf{H}$-NMR $\left(400 \mathrm{MHz}, \mathrm{CDCl}_{3}\right) \delta 7.94(\mathrm{ddd}, J=7.9,1.5,0.5$ $\mathrm{Hz}, 0 \mathrm{H}), 7.62(\mathrm{dd}, J=7.5,1.4 \mathrm{~Hz}, 1 \mathrm{H}), 7.48(\mathrm{td}, J=7.6,1.5 \mathrm{~Hz}, 1 \mathrm{H}), 7.40(\mathrm{td}, J=7.6,1.4 \mathrm{~Hz}$, 1H), 3.93 (s, 4H), 3.39 (s, 1H); ${ }^{13}$ C-NMR (101 MHz, $\left.\mathrm{CDCl}_{3}\right) \delta 166.57,135.10,132.64,131.85$, $130.41,128.62,122.78,82.36,82.14,52.32$.

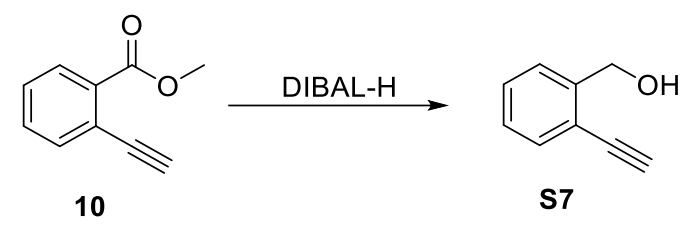

(2-Ethynylphenyl)methanol (S7). To a solution of terminal alkyne $\mathbf{1 0}$ (2.72 g, $16.9 \mathrm{mmol})$ in THF (100 mL) was slowly added DIBAL-H (1.2 M in toluene, $35.4 \mathrm{~mL}, 42.5 \mathrm{mmol})$ at $-78^{\circ} \mathrm{C}$. The reaction was stirred for $3 \mathrm{~h}$ at $-78^{\circ} \mathrm{C}$. Rochelle salt solution $(300 \mathrm{~mL})$ and ether $(200 \mathrm{~mL})$ were added and the mixture was vigorously stirred overnight. The aqueous layer was extracted with ether $\left(3 \times 150 \mathrm{~mL}\right.$ ) and the combined organic layers were dried over $\mathrm{MgSO}_{4}$ and concentrated under reduced pressure. Purification by FC ( $n$-hexane/EtOAc 20:1 $\rightarrow$ 4:1) yielded $1.59 \mathrm{~g}$ (12.0 mmol, 71\%) of alcohol $\mathbf{S 7 ^ { 5 }}$ as a yellow solid.

$\mathbf{R}_{\mathbf{f}}\left(n\right.$-hexane/EtOAc 4:1) $=0.22 ;{ }^{1} \mathbf{H}-\mathbf{N M R}\left(400 \mathrm{MHz}, \mathrm{CDCl}_{3}\right) \delta 7.51(\mathrm{dd}, J=7.6,1.3 \mathrm{~Hz}, 1 \mathrm{H})$, $7.48-7.41(\mathrm{~m}, 1 \mathrm{H}), 7.37(\mathrm{td}, J=7.6,1.4 \mathrm{~Hz}, 1 \mathrm{H}), 7.26(\mathrm{dd}, J=7.5,1.4 \mathrm{~Hz}, 1 \mathrm{H}), 4.84(\mathrm{~s}, 2 \mathrm{H})$, $3.34(\mathrm{~s}, 1 \mathrm{H}), 2.14(\mathrm{~s}, 1 \mathrm{H}) ;{ }^{13} \mathrm{C}-\mathbf{N M R}\left(101 \mathrm{MHz}, \mathrm{CDCl}_{3}\right) \delta 143.35,133.01,129.38,127.59$, 127.47, 120.37, 82.09, 81.39, 63.96. IR (film): $\tilde{v}=3289,3065,2878,2102,1480,1449,1206$, 1099, 1038, 1006, 947, 756, 711, 651, 611, 562, 446; HR-MS (EI): $\mathrm{m} / z$ calc. for $\mathrm{C}_{9} \mathrm{H}_{8} \mathrm{O}$ [M$\mathrm{H} \cdot]^{+}:$131.0502, found: 131.0482 .

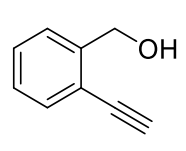

S7

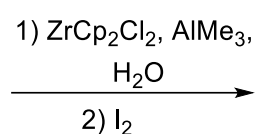

2) $\mathrm{I}_{2}$

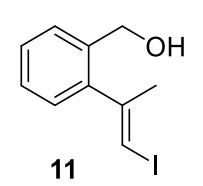

(E)-(2-(1-Iodoprop-1-en-2-yl)phenyl)methanol (11). ${ }^{6} \mathrm{AlMe}_{3}(2 \mathrm{M}$ in toluene, $22.4 \mathrm{~mL}$, $44.8 \mathrm{mmol}$ ) was added to a pale yellow solution of zirconocene dichloride (512 $\mathrm{mg}, 2.24 \mathrm{mmol}$ ) in $\mathrm{CH}_{2} \mathrm{Cl}_{2}(25 \mathrm{~mL})$ over $3 \mathrm{~min}$ at $0{ }^{\circ} \mathrm{C}$. The obtained yellowish solution was stirred for $30 \mathrm{~min}$ at room temperature and then cooled to $-78{ }^{\circ} \mathrm{C}$. Water $(202 \mu \mathrm{L}, 11.2 \mathrm{mmol})$ was added at -78 ${ }^{\circ} \mathrm{C}$ and the mixture was stirred for $30 \mathrm{~min}$ at $-78{ }^{\circ} \mathrm{C}$ and then $30 \mathrm{~min}$ at room temperature. A solution of alkyne $\mathbf{S 7}(1.48 \mathrm{~g}, 11.2 \mathrm{mmol})$ in $\mathrm{CH}_{2} \mathrm{Cl}_{2}(35 \mathrm{~mL})$ was added dropwise over $3 \mathrm{~min}$ at $-78{ }^{\circ} \mathrm{C}$. The obtained orange solution was stirred at room temperature for $3.5 \mathrm{~h}$. It was then cooled again to $-78{ }^{\circ} \mathrm{C}$ and a solution of iodine $(5.68 \mathrm{~g}, 22.4 \mathrm{mmol})$ in THF (25 mL) was added dropwise over $5 \mathrm{~min}$. The resulting red suspension was stirred for $30 \mathrm{~min}$ at $-78{ }^{\circ} \mathrm{C}$ and then 
warmed to $-10{ }^{\circ} \mathrm{C}$. The suspension was further warmed to room temperature during $1.5 \mathrm{~h}$ forming a yellow solution. The reaction was carefully quenched with by adding sat. aq. $\mathrm{Na}_{2} \mathrm{~S}_{2} \mathrm{O}_{3}$ solution (ca. $6 \mathrm{~mL}$ ) at $0{ }^{\circ} \mathrm{C}$. Caution: highly exothermic reaction! The slightly yellow suspension was slowly warmed to room temperature. Ether $(300 \mathrm{~mL})$ was added and the organic layer was dried over $\mathrm{MgSO}_{4}$. The suspension was filtered, the filtrate was concentrated under reduced pressure and the residue purified by FC ( $n$-hexane/EtOAc 6:1) to obtain $2.58 \mathrm{~g}(9.41$ mmol, $84 \%$ ) of vinyl iodide $\mathbf{1 1}^{6}$ as a yellowish oil.

$\mathbf{R}_{\mathbf{f}}\left(n\right.$-hexane/ EtOAc 6:1): 0.29; ${ }^{1} \mathbf{H}-\mathbf{N M R}\left(400 \mathrm{MHz}, \mathrm{CDCl}_{3}\right) \delta 7.47(\mathrm{dd}, J=7.5,1.1 \mathrm{~Hz}, 1 \mathrm{H})$, $7.32(\mathrm{td}, J=7.5,1.6 \mathrm{~Hz}, 1 \mathrm{H}), 7.27(\mathrm{td}, J=7.4,1.5 \mathrm{~Hz}, 1 \mathrm{H}), 7.11(\mathrm{dd}, J=7.5,1.5 \mathrm{~Hz}, 1 \mathrm{H}), 6.19$ (q, $J=1.1 \mathrm{~Hz}, 1 \mathrm{H}), 4.62(\mathrm{~s}, 2 \mathrm{H}), 2.18(\mathrm{~d}, J=1.2 \mathrm{~Hz}, 3 \mathrm{H}), 1.73(\mathrm{~s}, 1 \mathrm{H}) ;{ }^{13} \mathrm{C}-\mathbf{N M R}(101 \mathrm{MHz}$, $\left.\mathrm{CDCl}_{3}\right) \delta 147.60,141.76,137.41,128.44,128.10,127.83,80.12,62.97,26.92$. IR (film): $\tilde{v}=$ 3308, 3060, 3022, 2910, 2880, 1735, 1721, 1710, 1601, 1482, 1445, 1433, 1372, 1289, 1258, 1202, 1186, 1110, 1034, 1005, 946, 758, 723, 661, 605, 567, 556, 511; HR-MS (EI): $\mathrm{m} / \mathrm{z}$ calc. for $\mathrm{C}_{10} \mathrm{H}_{11} \mathrm{IO}[\mathrm{M} \cdot]^{+}:$273.9855, found: 273.9850 .

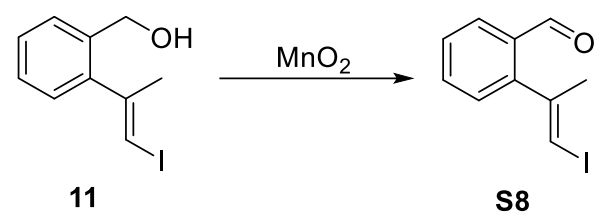

(E)-2-(1-Iodoprop-1-en-2-yl)benzaldehyde (S8). To a solution of benzylic alcohol 11 (500 $\mathrm{mg}, 1.82 \mathrm{mmol}$ ) in $\mathrm{CH}_{2} \mathrm{Cl}_{2}(50 \mathrm{~mL})$ was added $\mathrm{MnO}_{2}$ (activated, $1.59 \mathrm{~g}, 18.2 \mathrm{mmol}$ ). The reaction was stirred for $18 \mathrm{~h}$ at room temperature. The suspension was filtered and the filtrate rapidly concentrated under reduced pressure. The obtained aldehyde S8 was used immediately in the next step without purification.

${ }^{1} \mathbf{H}-\mathbf{N M R}\left(400 \mathrm{MHz}, \mathrm{CDCl}_{3}\right) \delta 10.09(\mathrm{~d}, J=0.6 \mathrm{~Hz}, 1 \mathrm{H}), 7.92(\mathrm{dd}, J=7.8,1.4 \mathrm{~Hz}, 1 \mathrm{H}), 7.56$ $(\mathrm{td}, J=7.6,1.5 \mathrm{~Hz}, 1 \mathrm{H}), 7.44(\mathrm{tt}, J=7.5,1.1 \mathrm{~Hz}, 1 \mathrm{H}), 7.28(\mathrm{dd}, J=7.6,1.2 \mathrm{~Hz}, 1 \mathrm{H}), 6.26(\mathrm{q}$, $J=1.2 \mathrm{~Hz}, 1 \mathrm{H}), 2.28(\mathrm{~d}, J=1.2 \mathrm{~Hz}, 3 \mathrm{H}) ;{ }^{13} \mathbf{C}-\mathbf{N M R}\left(101 \mathrm{MHz}, \mathrm{CDCl}_{3}\right) \delta 191.52,146.15$, $145.47,133.88,133.50,128.72,128.62,128.29,82.36,27.41$. 
<smiles>C/C(=C\I)c1ccccc1C=O</smiles>

S8

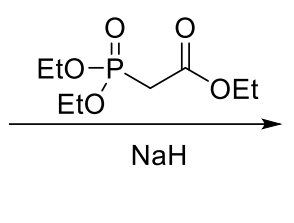<smiles>CCOC(=O)/C=C/c1ccccc1/C(C)=C/I</smiles>

Ethyl (E)-3-(2-((E)-1-iodoprop-1-en-2-yl)phenyl)acrylate (12). To a solution of triethyl phosphonoacetate $(816 \mathrm{mg}, 3.64 \mathrm{mmol})$ in THF $(15 \mathrm{~mL})$ was added sodium hydride $(60 \%$ in mineral oil, $132 \mathrm{mg}, 3.28 \mathrm{mmol}$ ) at $0{ }^{\circ} \mathrm{C}$. The solution was stirred for $1 \mathrm{~h}$ at room temperature before being added to a solution of the crude aldehyde $\mathbf{S 8}$ (ca. $495 \mathrm{mg}, 1.82 \mathrm{mmol}$ ) in THF (15 $\mathrm{mL}$ ) at $0{ }^{\circ} \mathrm{C}$. The mixture was stirred at room temperature for $24 \mathrm{~h} . *$ The mixture was poured on celite, the solvent was evaporated and the residue purified by FC ( $n$-hexane/EtOAc 20:1). The product still contained some aldehyde according to NMR analysis. Therefore, it was treated with another equivalent of deprotonated triethyl phosphonoacetate according to the procedure described above. The solution was again evaporated on celite and re-purified by FC ( $n$ hexane/EtOAc 20:1). The ester 12 was obtained as a yellowish oil (620 mg, $1.81 \mathrm{mmol}, 99 \%$ over two steps).

*TLC (n-hexane/EtOAc 15:1) did not separate the product from the starting material but dinitrophenylhydrazine stain suggested the absence of significant amounts of an aldehyde.

$\mathbf{R}_{\mathbf{f}}\left(n\right.$-hexane/EtOAc 15:1) $=0.38 ;{ }^{1} \mathbf{H}-\mathbf{N M R}\left(400 \mathrm{MHz}, \mathrm{CDCl}_{3}\right) \delta 7.73(\mathrm{~d}, J=15.9 \mathrm{~Hz}, 1 \mathrm{H})$, $7.63-7.56(\mathrm{~m}, 1 \mathrm{H}), 7.32(\mathrm{pd}, J=7.3,1.7 \mathrm{~Hz}, 2 \mathrm{H}), 7.21-7.15(\mathrm{~m}, 1 \mathrm{H}), 6.37(\mathrm{~d}, J=15.9 \mathrm{~Hz}$, $1 \mathrm{H}), 6.20(\mathrm{q}, J=1.2 \mathrm{~Hz}, 1 \mathrm{H}), 4.26(\mathrm{q}, J=7.1 \mathrm{~Hz}, 2 \mathrm{H}), 2.19(\mathrm{~d}, J=1.2 \mathrm{~Hz}, 3 \mathrm{H}), 1.34(\mathrm{t}, J=7.1$ $\mathrm{Hz}, 3 \mathrm{H}) ;{ }^{13} \mathrm{C}-\mathbf{N M R}\left(101 \mathrm{MHz}, \mathrm{CDCl}_{3}\right) \delta 166.88,147.08,143.61,142.70,132.05,129.99$, 128.59, 128.14, 126.95, 119.78, 81.93, 60.69, 27.04, 14.45; IR (film): $\tilde{v}=3060,2979,2931$, 2870, 2852, 17008, 1633, 1596, 1476, 1445, 1365, 1312, 1264, 1206, 1172, 1095, 1035, 983 , 862, 793, 755, 660, 597, 553, 522, 472; HR-MS (ESI): $m / z$ calc. for $\mathrm{C}_{14} \mathrm{H}_{16} \mathrm{IO}_{2}[\mathrm{M}+\mathrm{H}]^{+}$: 343.0190, found: 343.0187 . 


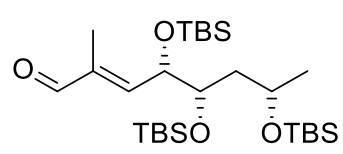

8

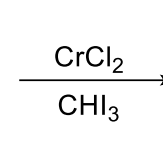

$\mathrm{HI}_{3}$

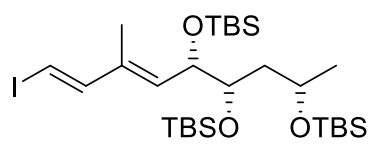

13

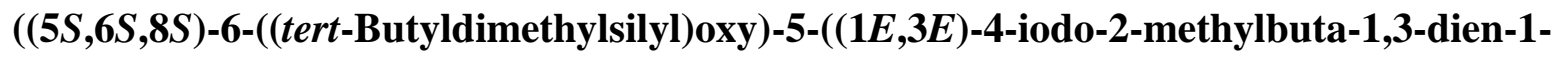
yl)-2,2,3,3,8,10,10,11,11-nonamethyl-4,9-dioxa-3,10-disiladodecane (13). To a stirred solution of $\mathrm{CrCl}_{2}(1.18 \mathrm{~g}, 9.61 \mathrm{mmol})$ in anhydrous THF $(10 \mathrm{~mL})$ was added a solution of aldehyde $\mathbf{8}^{2,7}$ (850 mg, $\left.1.60 \mathrm{mmol}\right)$ and $\mathrm{CHI}_{3}(1.89 \mathrm{~g}, 4.80 \mathrm{mmol})$ in anhydrous THF (5.5 mL $+2 \mathrm{~mL}$ for rinsing) dropwise. The reaction was stirred at room temperature for $15 \mathrm{~h}$. The reaction was quenched by the addition of water $(150 \mathrm{~mL})$ and the mixture was diluted with ether. The aqueous layer was extracted with ether $(3 \times 100 \mathrm{~mL})$ and the combined organic extracts were washed with sat. aq. $\mathrm{Na}_{2} \mathrm{~S}_{2} \mathrm{O}_{3}$, water and brine, dried over $\mathrm{MgSO}_{4}$, filtered, and concentrated under reduced pressure. The crude product was purified twice by FC ( $n$ hexane/EtOAc 100:1 in both cases) to afford the product (561 mg, pure fraction and $246 \mathrm{mg}$, impure fraction) as a colorless oil. The impure fraction was re-purified ( $n$-hexane/toluene 30:1) to obtain another $170 \mathrm{mg}$ (total: $731 \mathrm{mg}, 1.12 \mathrm{mmol}, \mathbf{7 0 \%}$ ) of vinyl iodide $\mathbf{1 3}$ as a colorless oil, that slowly decomposes upon storage at $-18{ }^{\circ} \mathrm{C}$.

$\mathbf{R}_{\mathbf{f}}(n$-hexane $)=0.14 .[\boldsymbol{\alpha}]^{\mathbf{D}_{\mathbf{2 0}}}=-27.94^{\circ}\left(\mathrm{c}=0.68, \mathrm{CHCl}_{3}\right) .{ }^{\mathbf{1}} \mathbf{H}-\mathbf{N M R}\left(400 \mathrm{MHz}, \mathrm{CDCl}_{3}\right) \delta 7.06$ $(\mathrm{dd}, J=14.6,0.6 \mathrm{~Hz}, 1 \mathrm{H}), 6.25(\mathrm{~d}, J=14.6 \mathrm{~Hz}, 1 \mathrm{H}), 5.47$ (d, $J=9.1 \mathrm{~Hz}, 1 \mathrm{H}), 4.37$ (dd, $J=9.1$, $3.7 \mathrm{~Hz}, 1 \mathrm{H}), 3.90(\mathrm{ddt}, J=11.4,8.1,5.9 \mathrm{~Hz}, 1 \mathrm{H}), 3.61(\mathrm{dt}, J=7.9,3.9 \mathrm{~Hz}, 1 \mathrm{H}), 1.81$ (ddd, $J=$ $13.3,8.2,4.0 \mathrm{~Hz}, 1 \mathrm{H}), 1.75$ (d, $J=1.2 \mathrm{~Hz}, 3 \mathrm{H}), 1.57$ (ddd, $J=13.6,8.5,5.4 \mathrm{~Hz}, 1 \mathrm{H}), 1.13$ (d, $J=6.0 \mathrm{~Hz}, 3 \mathrm{H}), 0.88(\mathrm{~s}, 9 \mathrm{H}), 0.86(\mathrm{~s}, 9 \mathrm{H}), 0.86(\mathrm{~s}, 9 \mathrm{H}), 0.05(\mathrm{~s}, 3 \mathrm{H}), 0.05(\mathrm{~s}, 3 \mathrm{H}), 0.04(\mathrm{~s}, 3 \mathrm{H})$, 0.03 (s, 3H), 0.01 (s, 3H), -0.03 (s, 3H). ${ }^{13} \mathbf{C}-N M R\left(101 \mathrm{MHz}, \mathrm{CDCl}_{3}\right) \delta$ 149.78, 134.91, 133.91, 75.20, 73.63, 71.12, 66.15, 42.67, 26.08, 26.01, 25.93, 23.57, 18.31, 18.20, 18.08, 13.12, -3.99, -4.17, -4.38, -4.43, -4.57. IR (film): $\tilde{v}=2954,2927,2884,2857,1472,1561$, 1250, 1091, 1068, 1004, 831, 807, 770, 663. HR-MS (ESI): $m / z$ calc. for $\mathrm{C}_{28} \mathrm{H}_{59} \mathrm{IO}_{3} \mathrm{Si}_{3} \mathrm{Na}$ $[\mathrm{M}+\mathrm{Na}]^{+}:$677.2709, found: 677.2700 . 


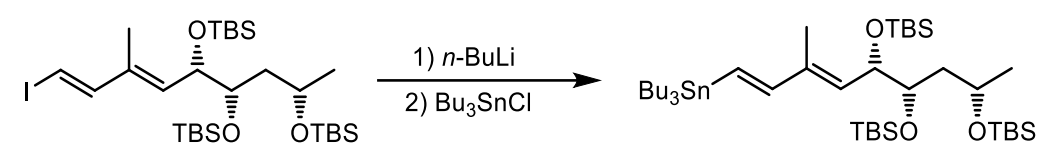

13

14

$(5 S, 6 S, 8 S)-6-((t e r t-B u t y l d i m e t h y l s i l y l) o x y)-2,2,3,3,8,10,10,11,11-n o n a m e t h y l-5-((1 E, 3 E)-$ 2-methyl-4-(tributylstannyl)buta-1,3-dien-1-yl)-4,9-dioxa-3,10-disiladodecane (14). To a stirred solution of vinyl iodide 13 (372 mg, $568 \mu \mathrm{mol})$ in diethyl ether (2 mL) was slowly added $n$-BuLi (1.6 $\mathrm{M}$ in hexane, $533 \mu \mathrm{L}, 852 \mu \mathrm{mol})$ at $-78{ }^{\circ} \mathrm{C}$. The reaction was stirred for $30 \mathrm{~min}$ at $-78^{\circ} \mathrm{C}$ before tributyltin chloride $(231 \mu \mathrm{l}, 852 \mu \mathrm{mol})$ was added. Stirring was continued for $1 \mathrm{~h}$ at $-78^{\circ} \mathrm{C}$, the mixture was warmed to room temperature within $1 \mathrm{~h}$ and stirred for $20 \mathrm{~min}$ at room temperature. Sat. aq. $\mathrm{NaHCO}_{3}$ solution $(5 \mathrm{~mL})$ was added and the aqueous layer was extracted with ether $(3 \times 25 \mathrm{~mL})$. The combined organic extracts were washed with brine, dried over $\mathrm{MgSO}_{4}$ and the solvent was evaporated under reduced pressure to obtain stannane $\mathbf{1 4}^{8}$ as a yellow oil (436 mg, $533 \mathrm{mmol}$, 94\% crude yield, contains residual $\mathrm{Bu}_{3} \mathrm{SnX}$ ). Since an attempt to purify a part of the product by $\mathrm{FC}\left(\mathrm{Et}_{3} \mathrm{~N}\right.$-buffered silica, $n$-hexane, $\left.1 \% \mathrm{Et}_{3} \mathrm{~N}\right)$ did not increase purity significantly, the crude material was used in the subsequent Stille coupling reactions without further purification. In contrast to vinyl iodide 13, stannane 14 proved to be storage stable at $-18{ }^{\circ} \mathrm{C}$ for extended periods.

\section{$\underline{2.4 .3 \text { Assembly of building blocks } 12 \text { and } 14 \text { by Stille coupling and ester cleavage }}$}

For the optimization of Stille coupling conditions, see Table S1, section 2.7

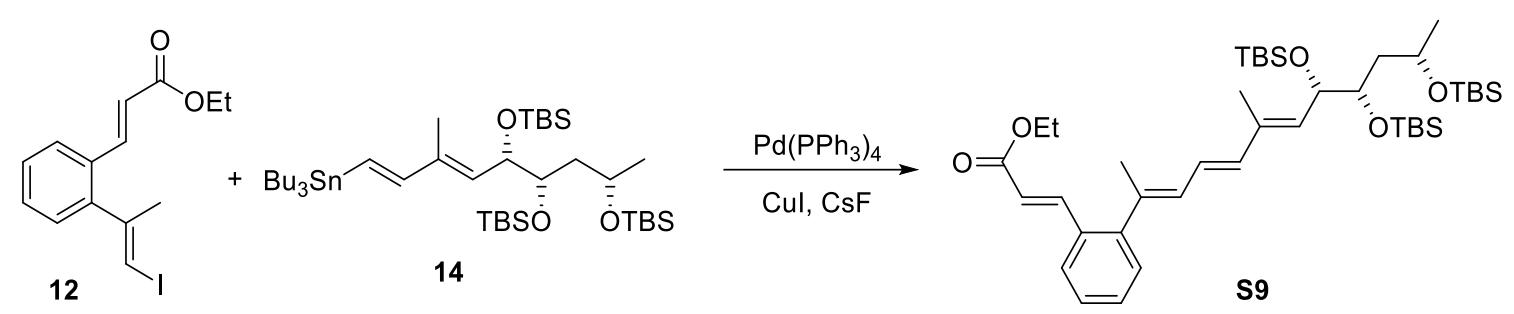

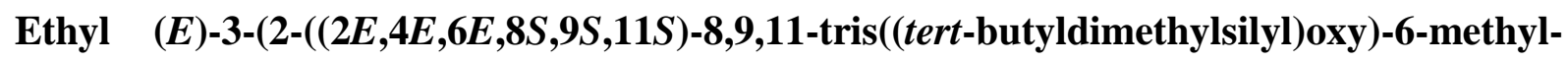
dodeca-2,4,6-trien-2 yl)phenyl)acrylate (S9). To vinyl iodide $12(20.0 \mathrm{mg}, 58.4 \mu \mathrm{mol})$ and stannane 14 (59.8 mg, $73.1 \mu \mathrm{mol})$ was added CsF (17.8 mg, $117 \mu \mathrm{mol})$, CuI (22.3 mg, 117 $\mu \mathrm{mol})$ and $\mathrm{Pd}\left(\mathrm{PPh}_{3}\right)_{4}(6.8 \mathrm{mg}, 5.8 \mu \mathrm{mol})$ in the glovebox and the reaction vial was sealed with a septum cap. Dry and degassed DMF ( $2 \mathrm{~mL}$ ) was added via cannula. The reaction was stirred at room temperature for $2.5 \mathrm{~h}$. Water $(15 \mathrm{~mL})$ and $n$-hexane $(60 \mathrm{~mL})$ were added and the organic 
layer was extracted with water $(2 \times 15 \mathrm{~mL})$ and brine $(15 \mathrm{~mL})$. The organic layer was dried over $\mathrm{MgSO}_{4}$, filtered and the volatiles removed under reduced pressure. The product was purified by FC ( $n$-hexane/EtOAc $150: 1)$ to obtain $38.8 \mathrm{mg}(52.2 \mu \mathrm{mol}, 89 \%)$ of the fully protected side chain $\mathbf{S 9}$ as a yellowish oil.

$\mathbf{R f}_{\mathbf{f}}\left(n\right.$-hexane/EtOAc 50:1) $=0.14 ;[\boldsymbol{\alpha}] \mathbf{D}^{\mathbf{2 0}}=-10.78^{\circ}\left(\mathrm{c}=1.02, \mathrm{CHCl}_{3}\right) ;{ }^{1} \mathbf{H}-\mathbf{N M R}(400 \mathrm{MHz}$, $\left.\mathrm{CDCl}_{3}\right) \delta 7.84(\mathrm{~d}, J=16.0 \mathrm{~Hz}, 1 \mathrm{H}), 7.59(\mathrm{dd}, J=7.8,1.4 \mathrm{~Hz}, 1 \mathrm{H}), 7.34(\mathrm{td}, J=7.4,1.4 \mathrm{~Hz}$, $1 \mathrm{H}), 7.29-7.21(\mathrm{~m}, 2 \mathrm{H}), 6.54(\mathrm{dd}, J=15.2,10.9 \mathrm{~Hz}, 1 \mathrm{H}), 6.37(\mathrm{~d}, J=16.0 \mathrm{~Hz}, 1 \mathrm{H}), 6.30(\mathrm{~d}$, $J=15.2 \mathrm{~Hz}, 1 \mathrm{H}), 6.04(\mathrm{dd}, J=10.9,1.7 \mathrm{~Hz}, 1 \mathrm{H}), 5.53(\mathrm{~d}, J=9.1 \mathrm{~Hz}, 1 \mathrm{H}), 4.44(\mathrm{dd}, J=9.2$, $3.6 \mathrm{~Hz}, 1 \mathrm{H}), 4.24(\mathrm{q}, J=7.1 \mathrm{~Hz}, 2 \mathrm{H}), 3.96-3.87$ (m, 1H), 3.64 (dt, $J=7.9,4.0 \mathrm{~Hz}, 1 \mathrm{H}), 2.15$ $(\mathrm{d}, J=1.3 \mathrm{~Hz}, 3 \mathrm{H}), 1.88(\mathrm{~d}, J=1.2 \mathrm{~Hz}, 3 \mathrm{H}), 1.87-1.80(\mathrm{~m}, 1 \mathrm{H}), 1.68-1.57(\mathrm{~m}, 1 \mathrm{H}), 1.31(\mathrm{t}$, $J=7.1 \mathrm{~Hz}, 3 \mathrm{H}), 1.14(\mathrm{~d}, J=6.0 \mathrm{~Hz}, 3 \mathrm{H}), 0.88(\mathrm{~s}, 18 \mathrm{H}), 0.87$ (s, 9H), $0.06(\mathrm{~s}, 3 \mathrm{H}), 0.06(\mathrm{~s}, 3 \mathrm{H})$, $0.05(\mathrm{~s}, 3 \mathrm{H}), 0.04(\mathrm{~s}, 3 \mathrm{H}), 0.03$ (s, 3H), -0.02 (s, 3H); ${ }^{13} \mathbf{C}-\mathbf{N M R}\left(101 \mathrm{MHz}, \mathrm{CDCl}_{3}\right) \delta 167.18$, $146.54,143.96,138.81,135.79,134.68,133.47,132.29,132.15,129.86,128.84,127.22$, 126.93, 123.91, 118.84, 73.82, 71.48, 66.23, 60.52, 42.85, 26.08, 25.98, 23.64, 19.44, 18.31, 18.24, 18.11, 14.48, 13.66, -3.97, -4.16, -4.35, -4.43, -4.54; IR (film): $\tilde{v}=2952,2910,2875$, 2845, 1457, 1413, 1375, 1236, 1176, 1087, 1054, 1003, 972, 796, 725, 673; HR-MS (ESI): $m / z$ calc. for $\mathrm{C}_{42} \mathrm{H}_{74} \mathrm{NaO}_{5} \mathrm{Si}_{3}[\mathrm{M}+\mathrm{Na}]^{+}:$765.4736, found: 765.4728.

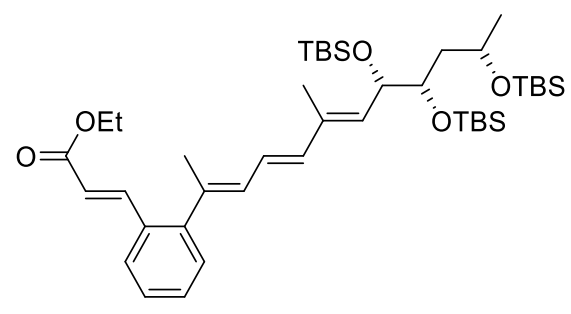

s9

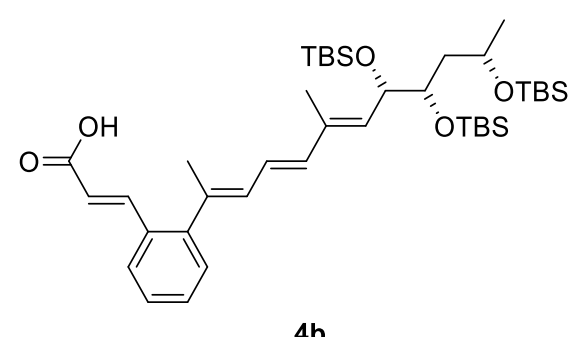

4b

\section{(E)-3-(2-((2E,4E,6E,8S,9S,11S)-8,9,11-tris((tert-Butyldimethylsilyl)oxy)-6-methyldodeca-}

2,4,6-trien-2-yl)phenyl)acrylic acid (4b). To a solution of ester $\mathbf{S 9}(40.0 \mathrm{mg}, 53.8 \mu \mathrm{mol})$ in $\mathrm{THF} / \mathrm{MeOH} / \mathrm{H}_{2} \mathrm{O}(4: 1: 1,4.5 \mathrm{~mL})$ was added $\mathrm{LiOH} \cdot \mathrm{H}_{2} \mathrm{O}(27.1 \mathrm{mg}, 646 \mu \mathrm{mol})$ at room temperature. The solution was protected from light and stirred for $12 \mathrm{~h}$ at room temperature. The reaction was quenched by adding $15 \mathrm{~mL}$ of sat. aq. $\mathrm{NH}_{4} \mathrm{Cl}$ solution and EtOAc. The layers were separated and the aqueous layer was extracted with EtOAc $(3 \times 25 \mathrm{~mL})$. The combined organic layers were washed with brine, dried over $\mathrm{MgSO}_{4}$ and concentrated under reduced pressure. Purification by FC ( $n$-hexane/EtOAc 8:1, $1 \% \mathrm{MeOH}, 0.01 \% \mathrm{HOAc}$ ) and subsequent co-evaporation of the combined fractions with toluene yielded $37.8 \mathrm{mg}(52.8 \mu \mathrm{mol}, 98 \%)$ of acid $\mathbf{4 b}$ as yellowish oil. 
$\mathbf{R}_{\mathbf{f}}(n$-hexane/EtOAc $4: 1)=0.40 ;[\boldsymbol{\alpha}]_{\mathbf{D}^{20}}=-14.28^{\circ}\left(\mathrm{c}=0.21, \mathrm{CHCl}_{3}\right) ;{ }^{1} \mathbf{H}-\mathbf{N M R}(400 \mathrm{MHz}$, $\left.\mathrm{CDCl}_{3}\right) \delta 7.92(\mathrm{~d}, J=15.9 \mathrm{~Hz}, 1 \mathrm{H}), 7.61(\mathrm{dd}, J=7.8,1.4 \mathrm{~Hz}, 1 \mathrm{H}), 7.36(\mathrm{td}, J=7.5,1.4 \mathrm{~Hz}$, $1 \mathrm{H}), 7.29(\mathrm{td}, J=7.6,1.5 \mathrm{~Hz}, 1 \mathrm{H}), 7.24(\mathrm{dd}, J=7.6,1.5 \mathrm{~Hz}, 1 \mathrm{H}), 6.53(\mathrm{dd}, J=15.2,10.9 \mathrm{~Hz}$, $1 \mathrm{H}), 6.38(\mathrm{~d}, J=15.9 \mathrm{~Hz}, 1 \mathrm{H}), 6.31(\mathrm{~d}, J=15.3 \mathrm{~Hz}, 1 \mathrm{H}), 6.04(\mathrm{dd}, J=11.0,1.7 \mathrm{~Hz}, 1 \mathrm{H}), 5.55$ $(\mathrm{d}, J=9.1 \mathrm{~Hz}, 1 \mathrm{H}), 4.44(\mathrm{dd}, J=9.2,3.6 \mathrm{~Hz}, 1 \mathrm{H}), 4.01-3.84(\mathrm{~m}, 1 \mathrm{H}), 3.65(\mathrm{dt}, J=8.0,4.0$ $\mathrm{Hz}, 1 \mathrm{H}), 2.15(\mathrm{~d}, J=1.4 \mathrm{~Hz}, 3 \mathrm{H}), 1.89$ (d, $J=1.2 \mathrm{~Hz}, 3 \mathrm{H}), 1.86-1.78(\mathrm{~m}, 1 \mathrm{H}), 1.61$ (ddd, $J=$ $13.3,7.8,5.3 \mathrm{~Hz}, 1 \mathrm{H}), 1.14(\mathrm{~d}, J=6.0 \mathrm{~Hz}, 3 \mathrm{H}), 0.88(\mathrm{~s}, 18 \mathrm{H}), 0.86(\mathrm{~s}, 9 \mathrm{H}), 0.06(\mathrm{~s}, 3 \mathrm{H}), 0.05$ (s, 3H), $0.05(\mathrm{~s}, 3 \mathrm{H}), 0.04(\mathrm{~s}, 3 \mathrm{H}), 0.02(\mathrm{~s}, 3 \mathrm{H}),-0.02(\mathrm{~s}, 3 \mathrm{H}) ;{ }^{13} \mathbf{C}-\mathbf{N M R}\left(101 \mathrm{MHz}, \mathrm{CDCl}_{3}\right) \delta$ $171.30,146.88,146.29,139.02,135.69,134.57,133.77,132.32,131.88,130.32,128.96$, 127.30, 127.17, 123.83, 117.66, 73.82, 71.53, 66.23, 42.94, 26.08, 25.98, 23.69, 19.47, 18.30, 18.23, 18.13, 13.63, -3.94, -3.99, -4.15, -4.35, -4.43, -4.52. IR (film): $\tilde{v}=2955,2928,2894$, 2885, 2857, 1691, 1627, 1473, 1461, 1416, 1387, 1368, 1314, 1255, 1209, 1143, 1068, 1004, 961, 937, 835, 808 774; HR-MS (ESI): $m / z$ calc. for $\mathrm{C}_{40} \mathrm{H}_{70} \mathrm{NaO}_{5} \mathrm{Si}_{3}[\mathrm{M}+\mathrm{Na}]^{+}:$737.4423, found: 737.4417 .

\subsection{Preparation of the side chain acid 4c}

$\underline{2.5 .1}$ Synthesis of the western vinyl iodide building block $\mathbf{1 8}$

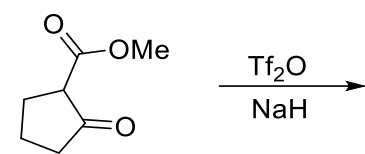

15

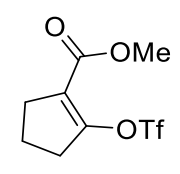

$\mathbf{S 1 0}$

Ethyl 2-(((trifluoromethyl)sulfonyl)oxy)cyclopent-1-enecarboxylate (S10). ${ }^{9}$ To a solution of methyl 2-oxocyclopentane-1-carboxylate $(\mathbf{1 5}, 4.00 \mathrm{~g}, 28.1 \mathrm{mmol})$ in $\mathrm{CH}_{2} \mathrm{Cl}_{2}(150 \mathrm{~mL})$ was slowly added $\mathrm{NaH}(60 \%$ dispersed in mineral oil, $1.35 \mathrm{~g}, 33.8 \mathrm{mmol})$ at $0{ }^{\circ} \mathrm{C}$. After stirring for $30 \mathrm{~min}$, trifluoromethanesulfonic anhydride $(5.68 \mathrm{~mL}, 33.8 \mathrm{mmol})$ was added dropwise to the reaction. The resulting mixture was then warmed to room temperature. After stirring overnight $(18 \mathrm{~h})$, the reagents were quenched by the addition of water. The mixture was then extracted with $\mathrm{CH}_{2} \mathrm{Cl}_{2}$. The combined organic layers were washed with water and brine, dried over $\mathrm{MgSO}_{4}$, filtered and concentrated under reduced pressure. The crude product was purified by FC (n-hexane/EtOAc 19:1) to obtain enol triflate $\mathbf{S 1 0}^{9}(6.54 \mathrm{~g}, 23.9 \mathrm{mmol}, 85 \%)$ as a colorless oil.

${ }^{\mathbf{1}} \mathbf{H}-\mathbf{N M R}\left(400 \mathrm{MHz}, \mathrm{CDCl}_{3}\right) \delta 3.79(\mathrm{~s}, 3 \mathrm{H}), 2.79-2.66(\mathrm{~m}, 4 \mathrm{H}), 2.02(\mathrm{p}, J=7.6 \mathrm{~Hz}, 2 \mathrm{H}) .{ }^{13} \mathbf{C}-$ NMR $\left(101 \mathrm{MHz}, \mathrm{CDCl}_{3}\right) \delta$ 162.82, 154.10, 77.16, 51.98, 32.91, 29.32, 18.97. 

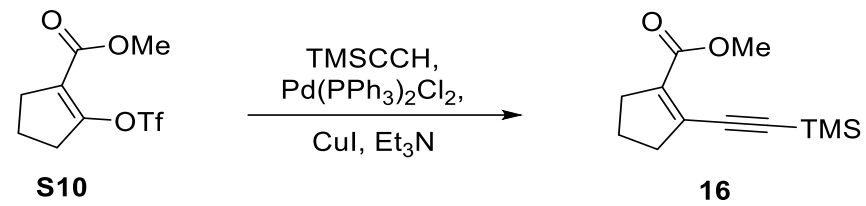

Methyl 2-((trimethylsilyl)ethynyl)cyclopent-1-ene-1-carboxylate (16). To $\mathrm{Pd}\left(\mathrm{PPh}_{3}\right)_{2} \mathrm{Cl}_{2}$ (102 mg, $146 \mu \mathrm{mol})$ and copper (I) iodide $(83.3 \mathrm{mg}, 483 \mu \mathrm{mol})$, a solution of enol triflate S10 (2.00 g, $7.29 \mathrm{mmol})$ in $30 \mathrm{~mL}$ THF was added via cannula followed by $\mathrm{NEt}_{3}(3.05 \mathrm{~mL}$, $21.1 \mathrm{mmol})$ and TMS-acetylene $(1.55 \mathrm{~mL}, 10.9 \mathrm{mmol})$. The suspension turned black and was stirred at ambient temperature for $70 \mathrm{~min}$. Sat. aq. $\mathrm{NH}_{4} \mathrm{Cl}(50 \mathrm{~mL})$ and ether $(300 \mathrm{~mL})$ were added and the organic layer was washed twice with sat. aq. $\mathrm{NH}_{4} \mathrm{Cl}(50 \mathrm{~mL})$ and brine. The solvent was removed under reduced pressure and FC (n-hexane/EtOAc 25:1) gave the TMSprotected alkyne $\mathbf{1 6}^{10}(1.59 \mathrm{~g}, 7.15 \mathrm{mmol}, 98 \%)$ as a brown oil.

$\mathbf{R}_{\mathbf{f}}\left(n\right.$-hexane/ EtOAc 25:1): 0.27. ${ }^{1} \mathbf{H}-\mathbf{N M R}\left(400 \mathrm{MHz}, \mathrm{CDCl}_{3}\right) \delta 3.76(\mathrm{~s}, 3 \mathrm{H}), 2.73-2.61(\mathrm{~m}$, 4H), 1.90 (p, $J=7.7 \mathrm{~Hz}, 2 \mathrm{H}), 0.23$ (s, 9H). ${ }^{13} \mathbf{C}-\mathbf{N M R}\left(101 \mathrm{MHz}, \mathrm{CDCl}_{3}\right) \delta 164.97,139.35$, 134.37, 106.15, 100.68, 51.48, 39.43, 33.45, 22.37, -0.05. IR (film): $\tilde{v}=2956,2899,2849$, 2144, 1704, 1607, 1435, 1352, 1249, 1203, 1121, 1031, 925, 838, 759, 714. HR-MS (ESI): $\mathrm{m} / \mathrm{z}$ calc. for $\mathrm{C}_{12} \mathrm{H}_{19} \mathrm{O}_{2} \mathrm{Si}[\mathrm{M}+\mathrm{H}]^{+}:$223.1149, found: 223.1152 .

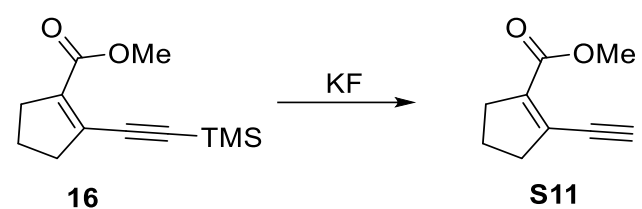

Ethyl 2-ethynylcyclopent-1-ene-1-carboxylate (S11). To a solution of KF (742 mg, $12.8 \mathrm{mmol})$ in $\mathrm{MeOH}(60 \mathrm{~mL})$ was added TMS-protected alkyne $16(1.42 \mathrm{~g}, 6.39 \mathrm{mmol})$ in THF $(15 \mathrm{~mL})$ at $0{ }^{\circ} \mathrm{C}$. The reaction was warmed to ambient temperature and stirred for $2.5 \mathrm{~h}$. Water $(75 \mathrm{~mL})$ and ether $(150 \mathrm{~mL})$ were added and the layers were separated. The organic layer was washed with water $(2 \times 50 \mathrm{~mL})$ and brine, dried over $\mathrm{MgSO}_{4}$ and the solvent evaporated under reduced pressure. FC ( $n$-hexane/EtOAc 15:1) furnished alkyne $\mathbf{S 1 1}^{10}$ (921 mg, 6.13 mmol, 96\%) as colorless crystals.

$\mathbf{R}_{\mathbf{f}}\left(n\right.$-hexane/ EtOAc 9:1): 0.35. ${ }^{1} \mathbf{H}$-NMR (400 MHz, $\left.\mathrm{CDCl}_{3}\right) \delta 3.77$ (s, 3H), $3.56(\mathrm{~s}, 1 \mathrm{H}), 2.75$ - $2.63(\mathrm{~m}, 4 \mathrm{H}), 1.94(\mathrm{p}, J=7.7 \mathrm{~Hz}, 2 \mathrm{H}) .{ }^{13} \mathbf{C}-\mathbf{N M R}\left(101 \mathrm{MHz}, \mathrm{CDCl}_{3}\right) \delta 164.81,140.07$, 133.85, 87.67, 87.67, 79.57, 51.65, 39.26, 33.38, 22.30. IR (film): $\tilde{v}=3227,3022,2967,2945$, 2915, 2854,2839, 2091, 1711, 1610, 1456, 1432, 1332, 1299, 1255, 1206, 1120, 1028, 915, 763, 711, 418. HR-MS (ESI): $m / z$ calc. for $\mathrm{C}_{9} \mathrm{H}_{11} \mathrm{O}_{2}[\mathrm{M}+\mathrm{H}]^{+}:$151.0754, found: 151.0754. 


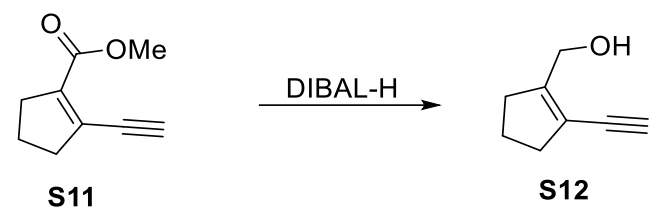

(2-Ethynylcyclopent-1-en-1-yl)methanol (S12). To ester S11 (910 mg, $6.06 \mathrm{mmol})$ in THF $(100 \mathrm{~mL})$ was slowly added DIBAL-H $\left(1.0 \mathrm{M}\right.$ in $\left.\mathrm{CH}_{2} \mathrm{Cl}_{2}, 15.2 \mathrm{~mL}, 15.2 \mathrm{mmol}\right)$ at $-78{ }^{\circ} \mathrm{C}$. The reaction was stirred for $4 \mathrm{~h}$ at $-78^{\circ} \mathrm{C}$. Sat. aq. Rochelle salt solution $(100 \mathrm{~mL})$ was added and the mixture was vigorously stirred for $2 \mathrm{~h}$. The aqueous layer was extracted with ether, and the combined organic layers were dried over $\mathrm{MgSO}_{4}$ and concentrated under reduced pressure. Purification by FC ( $n$-hexane/EtOAc 4:1) yielded $590 \mathrm{mg}(4.83 \mathrm{mmol}, 80 \%)$ of alcohol $\mathbf{S 1 2}$ as a yellow oil that slowly decomposes.

$\mathbf{R}_{\mathbf{f}}\left(n\right.$-hexane/ EtOAc 4:1): 0.32. ${ }^{1} \mathbf{H}$-NMR $\left(400 \mathrm{MHz} \mathrm{CDCl}_{3}\right) \delta 4.36(\mathrm{~s}, 2 \mathrm{H}), 3.18(\mathrm{~s}, 1 \mathrm{H}), 2.58$ - 2.45 (m, 4H), 1.91 (p, $J=7.6 \mathrm{~Hz}, 2 \mathrm{H}), 1.77$ (s, 1H). ${ }^{13} \mathbf{C}-\mathbf{N M R}\left(101 \mathrm{MHz}, \mathrm{CDCl}_{3}\right) \delta 151.84$, 119.00, 82.40, 79.77, 60.81, 37.00, 33.94, 22.50. IR (film): $\tilde{v}=3228,2950,2928,2852,2091$, $1631,1441,1243,1179,1030,1006,980,924,601,504$. HR-MS (EI): $\mathrm{m} / z$ calc. for $\mathrm{C}_{9} \mathrm{H}_{11} \mathrm{O}_{2}$ $[\mathrm{M} \cdot]^{+}:$122.0732, found: 122.0727 .

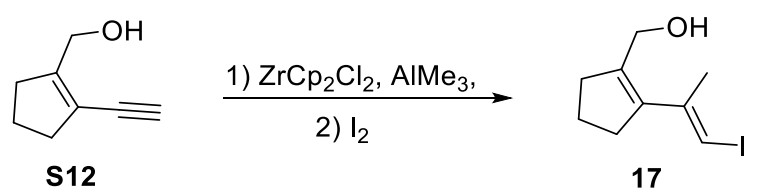

\section{(E)-(2-(1-Iodoprop-1-en-2-yl)cyclopent-1-en-1-yl)methanol (17)}

Procedure 1) $\mathrm{AlMe}_{3}(2 \mathrm{M}$ in toluene, $6.05 \mathrm{~mL}, 12.1 \mathrm{mmol}$ ) was added to a colorless solution of zirconocene dichloride $(276 \mathrm{mg}, 1.21 \mathrm{mmol})$ in $\mathrm{CH}_{2} \mathrm{Cl}_{2}(30 \mathrm{~mL})$ over $3 \mathrm{~min}$ at $0{ }^{\circ} \mathrm{C}$. The resulting pale yellow solution was stirred for $30 \mathrm{~min}$ at room temperature and cooled again to $0{ }^{\circ} \mathrm{C}$. A colorless solution of terminal alkyne $\mathbf{S 1 2}$ (590 mg, $4.82 \mathrm{mmol}$ ) in $\mathrm{CH}_{2} \mathrm{Cl}_{2}$ (15 mL) was added dropwise over $3 \mathrm{~min}$. The resulting yellow solution was stirred at room temperature for $40 \mathrm{~h}$, while it turned into an orange suspension. It was then cooled to $-78{ }^{\circ} \mathrm{C}$ and a solution of iodine $(1.47 \mathrm{~g}, 5.80 \mathrm{mmol})$ in THF $(10 \mathrm{~mL})$ was added dropwise over $10 \mathrm{~min}$. The resulting red suspension was stirred for $5 \mathrm{~min}$ at $-78{ }^{\circ} \mathrm{C}$, warmed to $-10{ }^{\circ} \mathrm{C}$ and then slowly to room temperature during $1.5 \mathrm{~h}$ to form a bright yellow solution. The reaction was very carefully quenched with by adding sat. aq. $\mathrm{Na}_{2} \mathrm{~S}_{2} \mathrm{O}_{3}$ solution $(5 \mathrm{~mL})$ at $0{ }^{\circ} \mathrm{C}$. Caution: highly exothermic reaction! An excess of $\mathrm{MgSO}_{4}$ was added for drying and the solids were removed by filtration and washed with $\mathrm{CH}_{2} \mathrm{Cl}_{2}$. The filtrate was concentrated under reduced pressure and purified by 
FC (n-hexane/ EtOAc 6:1) to obtain $758 \mathrm{mg}$ (2.87 mmol, 59\%) of vinyl iodide $\mathbf{1 7}$ as a brownish oil that slowly decomposes.

Procedure 2) $\mathrm{AlMe}_{3}(2 \mathrm{M}$ in toluene, $1.33 \mathrm{~mL}, 2.66 \mathrm{mmol}$ ) was added to a colorless solution of zirconocene dichloride $(187 \mathrm{mg}, 819 \mu \mathrm{mol})$ in $\mathrm{CH}_{2} \mathrm{Cl}_{2}(3 \mathrm{~mL})$ at $0{ }^{\circ} \mathrm{C}$ over $3 \mathrm{~min}$, giving a yellow solution. After stirring for $30 \mathrm{~min}$ at room temperature, terminal alkyne $\mathbf{S 1 2}(50.0 \mathrm{mg}$, $409 \mu \mathrm{mol})$ in $\mathrm{CH}_{2} \mathrm{Cl}_{2}(1 \mathrm{~mL})$ was added dropwise over $5 \mathrm{~min}$ at $0{ }^{\circ} \mathrm{C}$ to give a yellow solution. The latter was allowed to warm to room temperature followed by stirring for 3 days. The reaction was then cooled to $-78{ }^{\circ} \mathrm{C}$, and a solution of $\mathrm{I}_{2}(348 \mathrm{mg}, 1.37 \mathrm{mmol})$ in ether $(2 \mathrm{~mL})$ was added over $5 \mathrm{~min}$. The mixture was slowly warmed to $0{ }^{\circ} \mathrm{C}$ and then to room temperature while the color changed from brown to bright yellow. Quenching was very carefully performed using sat. aq. $\mathrm{Na}_{2} \mathrm{~S}_{2} \mathrm{O}_{3}$ solution $(5 \mathrm{~mL})$ at $0{ }^{\circ} \mathrm{C}$. Caution: highly exothermic reaction! The mixture was allowed to warm to room temperature and was then diluted with ether $(10 \mathrm{~mL})$ and water $(20 \mathrm{~mL})$. The aq. layer was extracted with ether $(3 \times 20 \mathrm{~mL})$. The combined organic layers were washed with water and brine $(20 \mathrm{~mL})$, dried over $\mathrm{MgSO}_{4}$ and concentrated under reduced pressure. Purification by FC ( $n$-hexane/EtOAc 6:1) afforded vinyl iodide 17 (48.2 mg, 183 $\mu \mathrm{mol}, 45 \%)$ as a yellow oil that slowly decomposes.

$\mathbf{R}_{\mathbf{f}}\left(n\right.$-hexane/ EtOAc 4:1): 0.40. ${ }^{1} \mathbf{H}$-NMR $\left(400 \mathrm{MHz}, \mathrm{CDCl}_{3}\right) \delta 6.01$ (q, $\left.J=1.1 \mathrm{~Hz}, 1 \mathrm{H}\right), 4.20$ (s, 2H), $2.55-2.45(\mathrm{~m}, 4 \mathrm{H}), 1.92(\mathrm{~d}, J=1.2 \mathrm{~Hz}, 3 \mathrm{H}), 1.85$ (p, $J=7.6 \mathrm{~Hz}, 2 \mathrm{H}), 1.54(\mathrm{~s}, 1 \mathrm{H})$. ${ }^{13}$ C-NMR $\left(101 \mathrm{MHz}, \mathrm{CDCl}_{3}\right) \delta 144.39,140.74,138.38,79.01,59.85,36.29,34.57,23.78$, 22.00. IR (film): $\tilde{v}=2956,2899,2849,1704,1607,1435,1352,1249,1203,1121,1031,925$, 838, 759, 714. HR-MS (EI): $\mathrm{m} / z$ calc. for $\mathrm{C}_{9} \mathrm{H}_{11} \mathrm{O}_{2}\left[\mathrm{M} \cdot-\mathrm{CH}_{3} \mathrm{O}\right]^{+}: 232.9827$, found: 232.9822 .

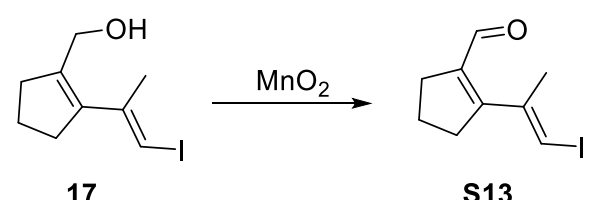

(E)-2-(1-Iodoprop-1-en-2-yl)cyclopent-1-ene-1-carbaldehyde (S13). To allylic alcohol 17 (33.0 mg, $125 \mu \mathrm{mol})$ in $\mathrm{CDCl}_{3}\left(1 \mathrm{~mL}\right.$ ) was added $\mathrm{MnO}_{2}$ (activated, $109 \mathrm{mg}, 1.25 \mathrm{mmol}$ ). The reaction was stirred for $20 \mathrm{~h}$ at room temperature. The suspension was filtered and used for NMR-characterization of the unstable product S13, which decomposes upon concentration. Therefore, the oxidation was performed in situ prior to HWE olefination for preparative purposes (see the next procedure, compound 18). 
${ }^{1} \mathbf{H}-\mathbf{N M R}\left(400 \mathrm{MHz}, \mathrm{CDCl}_{3}\right) \delta 9.78(\mathrm{~s}, 1 \mathrm{H}), 6.46(\mathrm{q}, J=1.2 \mathrm{~Hz}, 1 \mathrm{H}), 2.72(\mathrm{tt}, J=7.7,2.1 \mathrm{~Hz}$, 2H), 2.59 (tt, $J=7.7,2.1 \mathrm{~Hz}, 2 \mathrm{H}), 2.03$ (d, $J=1.2 \mathrm{~Hz}, 3 \mathrm{H}), 1.91$ (p, $J=7.7 \mathrm{~Hz}, 2 \mathrm{H}) .{ }^{13} \mathbf{C}-\mathbf{N M R}$ $\left(101 \mathrm{MHz}, \mathrm{CDCl}_{3}\right) \delta 189.43,162.81,142.61,141.03,83.95,37.65,30.52,23.62,21.70$.

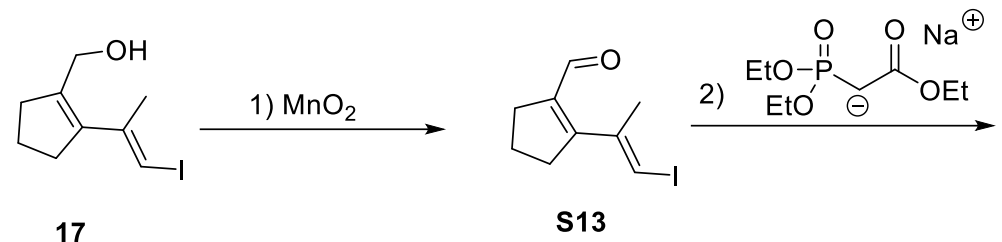

17
S13

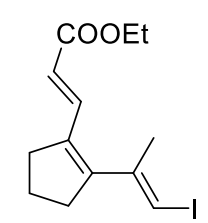

18

\section{Ethyl (E)-3-(2-((E)-1-iodoprop-1-en-2-yl)cyclopent-1-en-1-yl)acrylate (18)}

Step 1) To the allylic alcohol $17(150.0 \mathrm{mg}, 568 \mu \mathrm{mol})$ in THF $(15 \mathrm{~mL})$ was added $\mathrm{MnO}_{2}$ (activated, $494 \mathrm{mg}, 5.68 \mathrm{mmol}$ ). The reaction was stirred for $18 \mathrm{~h}$ at room temperature. The suspension containing aldehyde $\mathbf{S 1 3}$ was used in the next step without workup or purification.

Step 2) To a solution of triethyl phosphonoacetate $(253 \mathrm{mg}, 1.13 \mathrm{mmol})$ in THF (10 mL) was added sodium hydride ( $60 \%$ in mineral oil, $40.0 \mathrm{mg}, 1.02 \mathrm{mmol})$ at $0{ }^{\circ} \mathrm{C}$. The solution was stirred for $30 \mathrm{~min}$ at $0{ }^{\circ} \mathrm{C}$ and for $30 \mathrm{~min}$ at room temperature before being added to the crude suspension of aldehyde $\mathbf{S 1 3}$ (ca. $149 \mathrm{mg}, 568 \mu \mathrm{mol})$ in $\mathrm{THF}\left(15 \mathrm{~mL}\right.$ ) at $0{ }^{\circ} \mathrm{C}$. The mixture was stirred at room temperature for $4 \mathrm{~h}$. Celite was added, the mixture concentrated under reduced pressure and purified by FC ( $n$-hexane/EtOAc 25:1). Product 18 (176 mg, $530 \mu \mathrm{mol}, 93 \%$, two steps) was obtained as a yellow oil.

$\mathbf{R}_{\mathbf{f}}\left(n\right.$-hexane/EtOAc 25:1) $=0.35 ;{ }^{1} \mathbf{H}-\mathbf{N M R}\left(400 \mathrm{MHz}, \mathrm{CDCl}_{3}\right) \delta 7.56(\mathrm{~d}, J=15.6 \mathrm{~Hz}, 1 \mathrm{H})$, $6.20(\mathrm{~s}, 1 \mathrm{H}), 5.81(\mathrm{~d}, J=15.6 \mathrm{~Hz}, 1 \mathrm{H}), 4.20(\mathrm{q}, J=7.1 \mathrm{~Hz}, 2 \mathrm{H}), 2.61(\mathrm{t}, J=7.4 \mathrm{~Hz}, 2 \mathrm{H}), 2.53$ (t, $J=7.5 \mathrm{~Hz}, 2 \mathrm{H}), 1.96(\mathrm{~s}, 3 \mathrm{H}), 1.91(\mathrm{p}, J=7.7 \mathrm{~Hz}, 2 \mathrm{H}), 1.29(\mathrm{t}, J=7.1 \mathrm{~Hz}, 3 \mathrm{H}) ;{ }^{13} \mathbf{C}-\mathbf{N M R}$ $\left(101 \mathrm{MHz}, \mathrm{CDCl}_{3}\right) \delta 167.58,151.01,143.90,138.89,135.54,119.46,82.15,60.40,37.16$, 32.91, 23.81, 21.75, 14.43; IR (film): $\tilde{v}=3062$, 2976, 2953, 2843, 1704, 1616, 1463, 1442, 1365, 1304, 1262, 1243, 1158, 1095, 1066, 1036, 982, 916, 855, 788, 729, 676, 609, 562, 542, 502; HR-MS (ESI): $m / z$ calc. for $\mathrm{C}_{44} \mathrm{H}_{75} \mathrm{~N}_{4} \mathrm{O}_{12} \mathrm{~S}[\mathrm{M}+\mathrm{H}]^{+}: 333.0346$, found: 333.0349 . 
2.5.2 Assembly of the building blocks 18 and 14 by Stille coupling and ester cleavage

For the optimization of Stille coupling conditions, see Table S1, section 2.7

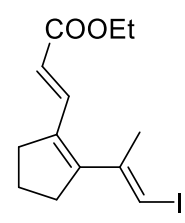

18

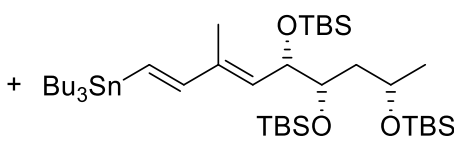

14

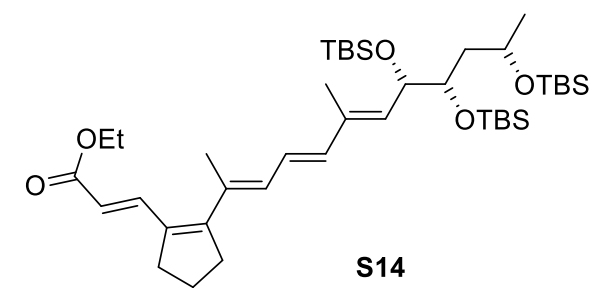

S14

Ethyl (E)-3-(2-((2E,4E,6E,8S,9S,11S)-8,9,11-tris((tert-Butyldimethylsilyl)-oxy)-6-methyldodeca-2,4,6-trien-2-yl)cyclopent-1-en-1-yl)acrylate (S14). To vinyl iodide 18 (40.0 mg, 120 $\mu \mathrm{mol})$ and stannane 14 (123 mg, $151 \mu \mathrm{mol})$ were added CsF (36.6 mg, $241 \mu \mathrm{mol}), \mathrm{CuI}(45.9$ $\mathrm{mg}, 241 \mu \mathrm{mol})$ and $\mathrm{Pd}\left(\mathrm{PPh}_{3}\right)_{4}(13.9 \mathrm{mg}, 12.0 \mu \mathrm{mol})$ in a glovebox and the reaction vial was sealed with a septum cap. Dry and degassed DMF (4 mL) was added via cannula. The reaction was stirred at room temperature for $3 \mathrm{~h}$. Water $(15 \mathrm{~mL})$, ether $(15 \mathrm{~mL})$ and $n$-hexane $(60 \mathrm{~mL})$ were added and the organic layer extracted with water $(2 \times 15 \mathrm{~mL})$ and brine $(15 \mathrm{~mL})$. The organic layer was dried over $\mathrm{MgSO}_{4}$, filtered and the volatiles were removed under reduced pressure. The product was purified twice by FC ( $n$-hexane/EtOAc 150:1 and toluene 100\%) to obtain $65.5 \mathrm{mg}(89.2 \mu \mathrm{mol}, 74 \%)$ of the fully protected side chain $\mathbf{S 1 4}$ as yellow oil.

$\mathbf{R f}_{\mathbf{f}}\left(n\right.$-hexane/EtOAc 20:1) $=0.42 ;[\boldsymbol{\alpha}]_{\mathbf{D}^{\mathbf{2 0}}}=-26.99^{\circ}\left(\mathrm{c}=1.00, \mathrm{CHCl}_{3}\right) ;{ }^{\mathbf{1}} \mathbf{H}-\mathbf{N M R}(400 \mathrm{MHz}$, $\left.\mathrm{CDCl}_{3}\right) \delta 7.77(\mathrm{~d}, J=15.5 \mathrm{~Hz}, 1 \mathrm{H}), 6.50(\mathrm{dd}, J=15.1,11.0 \mathrm{~Hz}, 1 \mathrm{H}), 6.35(\mathrm{~d}, J=15.2 \mathrm{~Hz}, 1 \mathrm{H})$, $6.16(\mathrm{~d}, J=11.0 \mathrm{~Hz}, 1 \mathrm{H}), 5.78(\mathrm{~d}, J=15.6 \mathrm{~Hz}, 1 \mathrm{H}), 5.55(\mathrm{~d}, J=9.1 \mathrm{~Hz}, 1 \mathrm{H}), 4.43(\mathrm{dd}, J=9.2$, $3.7 \mathrm{~Hz}, 1 \mathrm{H}), 4.20(\mathrm{q}, J=7.1 \mathrm{~Hz}, 2 \mathrm{H}), 3.96-3.86(\mathrm{~m}, 1 \mathrm{H}), 3.63(\mathrm{dt}, J=8.0,4.0 \mathrm{~Hz}, 1 \mathrm{H}), 2.66$ (t, $J=7.4 \mathrm{~Hz}, 2 \mathrm{H}), 2.58(\mathrm{t}, J=7.4 \mathrm{~Hz}, 2 \mathrm{H}), 2.00(\mathrm{~d}, J=1.2 \mathrm{~Hz}, 3 \mathrm{H}), 1.90(\mathrm{p}, J=7.5 \mathrm{~Hz}, 2 \mathrm{H})$, $1.85(\mathrm{~d}, J=1.2 \mathrm{~Hz}, 3 \mathrm{H}), 1.84-1.77(\mathrm{~m}, 1 \mathrm{H}), 1.60(\mathrm{ddd}, J=13.6,8.0,5.3 \mathrm{~Hz}, 1 \mathrm{H}), 1.29(\mathrm{t}, J=$ $7.1 \mathrm{~Hz}, 3 \mathrm{H}), 1.14(\mathrm{~d}, J=6.0 \mathrm{~Hz}, 3 \mathrm{H}), 0.88(\mathrm{~s}, 9 \mathrm{H}), 0.87$ (s, 9H), $0.86(\mathrm{~s}, 9 \mathrm{H}), 0.05$ (s, 6H), 0.05 (s, 3H), 0.03 (s, 3H), 0.02 (s, 3H), -0.03 (s, 3H); ${ }^{13}$ C-NMR (101 MHz, $\left.\mathrm{CDCl}_{3}\right) \delta 167.97,153.67$, 140.06, 139.37, 134.81, 134.36, 133.74, 133.30, 131.58, 123.80, 118.12, 73.81, 71.50, 66.23, $60.24,42.83,37.40,33.63,26.08,26.06,25.97,23.63,21.82,18.31,18.24,18.11,16.79,14.49$, 13.65, -3.98, -4.17, -4.36, -4.42, -4.54; IR (film): $\tilde{v}=2955,2929,2894,2857,1709,1615$, 1609, 1472, 1462, 1445, 1387, 1362, 1302, 1250, 1159, 1094, 1066, 1048, 1004, 961, 835, 808, 774; HR-MS (ESI): $m / z$ calc. for $\mathrm{C}_{41} \mathrm{H}_{76} \mathrm{NaO}_{5} \mathrm{Si}_{3}[\mathrm{M}+\mathrm{Na}]^{+}:$755.4893, found: 755.4883 . 

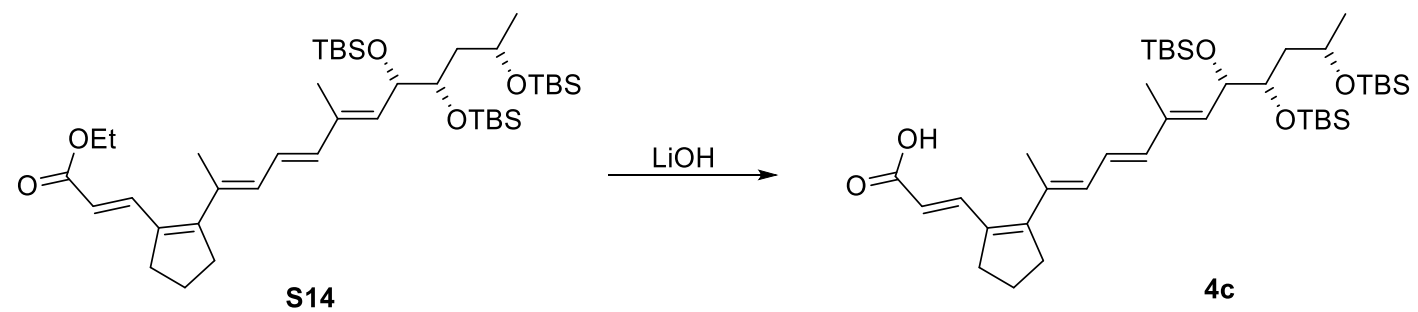

(E)-3-(2-((2E,4E,6E,8S,9S,11S)-8,9,11-tris((tert-Butyldimethylsilyl)oxy)-6-methyldodeca-

2,4,6-trien-2-yl)cyclopent-1-en-1-yl)acrylic acid (4c). To a solution of ester S14 (42.0 mg, $57.3 \mu \mathrm{mol})$ in $\mathrm{THF} / \mathrm{MeOH} / \mathrm{H}_{2} \mathrm{O}(4: 1: 1,2.3 \mathrm{~mL})$ was added $\mathrm{LiOH} \cdot \mathrm{H}_{2} \mathrm{O}(28.8 \mathrm{mg}, 687 \mu \mathrm{mol})$ at room temperature. The solution was protected from light and stirred for $14 \mathrm{~h}$ at room temperature. The reaction was quenched by adding $15 \mathrm{~mL}$ of sat. aq. $\mathrm{NH}_{4} \mathrm{Cl}$ solution and EtOAc $(20 \mathrm{~mL})$. The layers were separated and the aqueous layer was extracted with EtOAc $(3 \times 25$ $\mathrm{mL}$ ). The combined organic layers were washed with brine, dried over $\mathrm{MgSO}_{4}$ and concentrated under reduced pressure. Purification by FC ( $n$-hexane/EtOAc 8:1, 1\% MeOH, 0.01\% HOAc) and subsequent co-evaporation of the combined fractions with toluene yielded $38.7 \mathrm{mg}$ (54.9 $\mu \mathrm{mol}, \mathbf{9 6 \%}$ ) of acid $\mathbf{4 c}$ as a yellow oil.

$\mathbf{R f}_{\mathbf{f}}(n \text {-hexane/EtOAc 4:1) }=0.19 ;[\boldsymbol{\alpha}]]^{\mathbf{2 0}}=-30.76^{\circ}\left(\mathrm{c}=1.45, \mathrm{CHCl}_{3}\right) ;{ }^{1} \mathbf{H}-\mathbf{N M R}(400 \mathrm{MHz}$, $\left.\mathrm{CDCl}_{3}\right) \delta 7.86(\mathrm{~d}, J=15.5 \mathrm{~Hz}, 1 \mathrm{H}), 6.49(\mathrm{dd}, J=15.1,10.9 \mathrm{~Hz}, 1 \mathrm{H}), 6.36(\mathrm{~d}, J=15.1 \mathrm{~Hz}, 1 \mathrm{H})$, $6.18(\mathrm{~d}, J=11.0 \mathrm{~Hz}, 1 \mathrm{H}), 5.77(\mathrm{~d}, J=15.4 \mathrm{~Hz}, 1 \mathrm{H}), 5.56$ (d, $J=9.0 \mathrm{~Hz}, 1 \mathrm{H}), 4.43$ (dd, $J=9.2$, $3.6 \mathrm{~Hz}, 1 \mathrm{H}), 3.96-3.85(\mathrm{~m}, 1 \mathrm{H}), 3.64(\mathrm{dt}, J=8.0,4.0 \mathrm{~Hz}, 1 \mathrm{H}), 2.68(\mathrm{t}, J=7.4 \mathrm{~Hz}, 2 \mathrm{H}), 2.60$ (t, $J=7.5 \mathrm{~Hz}, 2 \mathrm{H}), 2.01(\mathrm{~s}, 3 \mathrm{H}), 1.93-1.87(\mathrm{~m}, 2 \mathrm{H}), 1.87-1.84(\mathrm{~m}, 3 \mathrm{H}), 1.84-1.79(\mathrm{~m}, 1 \mathrm{H})$, $1.61(\mathrm{ddd}, J=13.2,7.8,5.3 \mathrm{~Hz}, 1 \mathrm{H}), 1.14$ (d, $J=5.8 \mathrm{~Hz}, 3 \mathrm{H}), 0.88$ (s, 9H), 0.87 (s, 8H), 0.86 (s, 10H), 0.05 (s, 9H), 0.03 (s, 3H), 0.02 (s, 3H), -0.02 (s, 3H); ${ }^{13} \mathbf{C}-\mathbf{N M R}\left(101 \mathrm{MHz}, \mathrm{CDCl}_{3}\right)$ $\delta 172.53,154.92,142.18,139.73,134.70,134.34,134.11,133.20,132.00,123.74,117.05$, 73.81, 71.55, 66.22, 42.91, 37.52, 33.66, 26.08, 25.98, 23.67, 21.81, 18.30, 18.24, 18.13, 16.88, 13.64, $-3.95,-4.00,-4.16,-4.36,-4.43,-4.52$. IR (film): $\tilde{v}=2954,2928,2893,2856,1682$, 1602, 1472, 1462, 1412, 1387, 1379, 1361, 1305, 1275, 1251, 1196, 1143, 1129, 1093, 1068, 1004, 960, 938, 918, 896, 835, 773, 674, 664; HR-MS (ESI): $\mathrm{m} / z$ calc. for $\mathrm{C}_{39} \mathrm{H}_{72} \mathrm{NaO}_{5} \mathrm{Si}_{3}$ $[\mathrm{M}+\mathrm{Na}]^{+}:$727.4580, found: 727.4571 . 


\subsection{Esterification of the mycolactone core with acids $4 a-4 c$ and final deprotection}

Synthesis of mycolactone analog $\mathbf{2 a}$

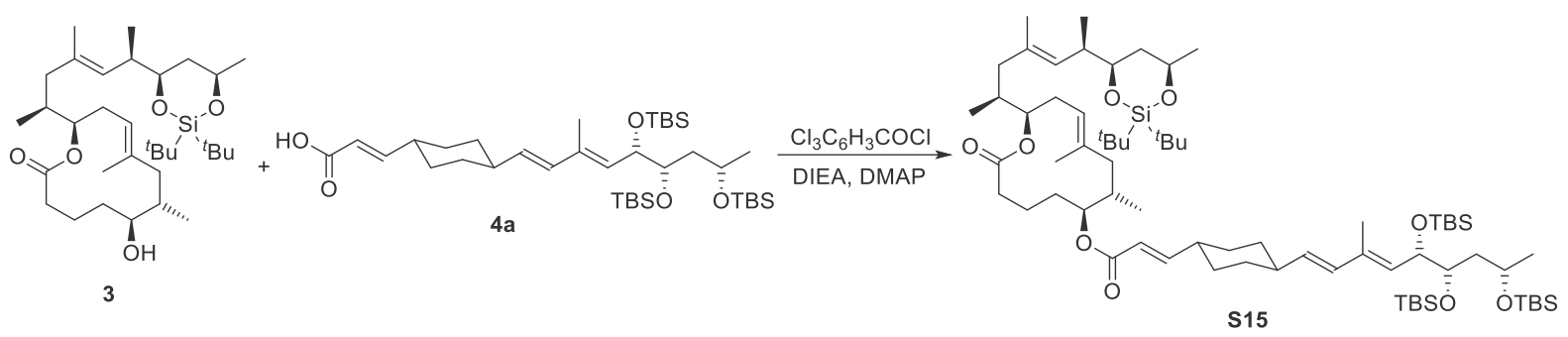

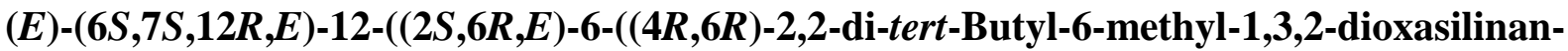
4-yl)-4-methylhept-4-en-2-yl)-7,9-dimethyl-2-oxooxacyclododec-9-en-6-yl 3-((1S,4S)-4((1E,3E,5S,6S,8S)-5,6,8-tris((tert-butyldimethylsilyl)oxy)-3-methylnona-1,3-dien-1-

yl)cyclohexyl)acrylate (S15). To a solution of acid $4 \mathbf{a}(42.0 \mathrm{mg}, 61.7 \mu \mathrm{mol})$ in THF $(1.5 \mathrm{~mL})$ were added DIEA (42.7 $\mu \mathrm{L}, 247 \mu \mathrm{mol}), 2$,4,6-trichlorobenzoyl chloride $(19.3 \mu \mathrm{L}, 123 \mu \mathrm{mol})$ and DMAP $(37.7 \mathrm{mg}, 308 \mu \mathrm{mol})$ at room temperature. Immediately, the initially colorless solution became turbid. After stirring at room temperature for $5 \mathrm{~min}$, a solution of alcohol $\mathbf{3}^{2}$ (20.9 $\mathrm{mg}, 37.0 \mu \mathrm{mol})$ in THF $(1.5 \mathrm{~mL})$ was added. The slightly yellow suspension was protected from light and stirred for $3 \mathrm{~h}$ at room temperature before the reaction was quenched by adding sat. aq. $\mathrm{NaHCO}_{3}$ solution $(10 \mathrm{~mL})$ and EtOAc $(20 \mathrm{~mL})$. The layers were separated and the aqueous layer was extracted with EtOAc $(2 \times 10 \mathrm{~mL})$. The combined organic layers were washed with brine $(3 \mathrm{~mL})$ and dried over $\mathrm{MgSO}_{4}$. Silica gel was added and the resulting suspension was concentrated under reduced pressure. Repeated purification by FC ( $n$ hexane/EtOAc 100:1 $\rightarrow$ 50:1 $\rightarrow$ 25:1; then $n$-hexane/EtOAc 50:1 $\rightarrow$ 40:1) yielded $41.0 \mathrm{mg}$ (33.4 $\mu \mathrm{mol}, 90 \%)$ of ester $\mathbf{S 1 5}$ as a colorless oil.

$\mathbf{R}_{\mathbf{f}}(n \text {-hexane/EtOAc 9:1) }=0.52 ;[\alpha]]_{\mathbf{D}^{25}}=-25.9^{\circ}\left(\mathrm{c}=1.03, \mathrm{CHCl}_{3}\right) ;{ }^{1} \mathbf{H}-\mathbf{N M R}(400 \mathrm{MHz}$, $\left.\mathrm{CDCl}_{3}\right): \delta 6.92(\mathrm{dd}, J=15.7,6.7 \mathrm{~Hz}, 1 \mathrm{H}), 6.03(\mathrm{~d}, J=15.8 \mathrm{~Hz}, 1 \mathrm{H}), 5.76(\mathrm{dd}, J=15.8,1.4 \mathrm{~Hz}$, 1H), 5.52 (dd, $J=15.7,6.9 \mathrm{~Hz}, 1 \mathrm{H}), 5.37$ (d, $J=9.1 \mathrm{~Hz}, 1 \mathrm{H}), 5.05$ (d, $J=11.0 \mathrm{~Hz}, 1 \mathrm{H}), 4.95$ $(\mathrm{d}, J=9.7 \mathrm{~Hz}, 1 \mathrm{H}), 4.88(\mathrm{ddd}, J=11.6,5.6,2.8 \mathrm{~Hz}, 1 \mathrm{H}), 4.78-4.69(\mathrm{~m}, 1 \mathrm{H}), 4.39(\mathrm{dd}, J=9.1$, $3.7 \mathrm{~Hz}, 1 \mathrm{H}), 4.13(\mathrm{ddd}, J=11.1,6.1,2.1 \mathrm{~Hz}, 1 \mathrm{H}), 3.96-3.84(\mathrm{~m}, 1 \mathrm{H}), 3.75-3.65(\mathrm{~m}, 1 \mathrm{H})$, $3.65-3.57(\mathrm{~m}, 1 \mathrm{H}), 2.50-2.25(\mathrm{~m}, 3 \mathrm{H}), 2.17-1.48(\mathrm{~m}, 30 \mathrm{H}), 1.41-1.09(\mathrm{~m}, 11 \mathrm{H}), 1.06-$ $0.77(\mathrm{~m}, 54 \mathrm{H}), 0.13--0.10(\mathrm{~m}, 18 \mathrm{H}) ;{ }^{13} \mathbf{C}-\mathbf{N M R}\left(100 \mathrm{MHz}, \mathrm{CDCl}_{3}\right): \delta 173.34,166.52,154.10$, 136.84, 134.29, 134.17, 132.98, 132.85, 130.56, 130.00, 122.74, 119.35, 78.91, 78.01, 76.15, 73.82, 71.52, 70.68, 66.29, 45.69, 43.57, 42.93, 41.86, 40.58, 40.56, 40.30, 35.61, 34.92, 32.55, $32.53,32.01,31.51,31.48,30.34,29.73,25.09$, 23.69, 22.86, 20.55, 19.73, 18.31, 18.24, 18.15, 
16.84, 16.22, 15.87, 14.64, 13.63, -3.97, -4.02, -4.14, -4.35, -4.42, -4.52; IR (film, EtOAc): $\widetilde{v}$ $=2930,2859,1728,1464,1378,1253,1152,1135,1098,986,944,890,833$, 776; HR-MS (ESI) calc. 1244.9130 for $\mathrm{C}_{70} \mathrm{H}_{134} \mathrm{NO}_{9} \mathrm{Si}_{4}{ }^{+}\left[\mathrm{M}+\mathrm{NH}_{4}\right]^{+}$, found 1244.9121 .
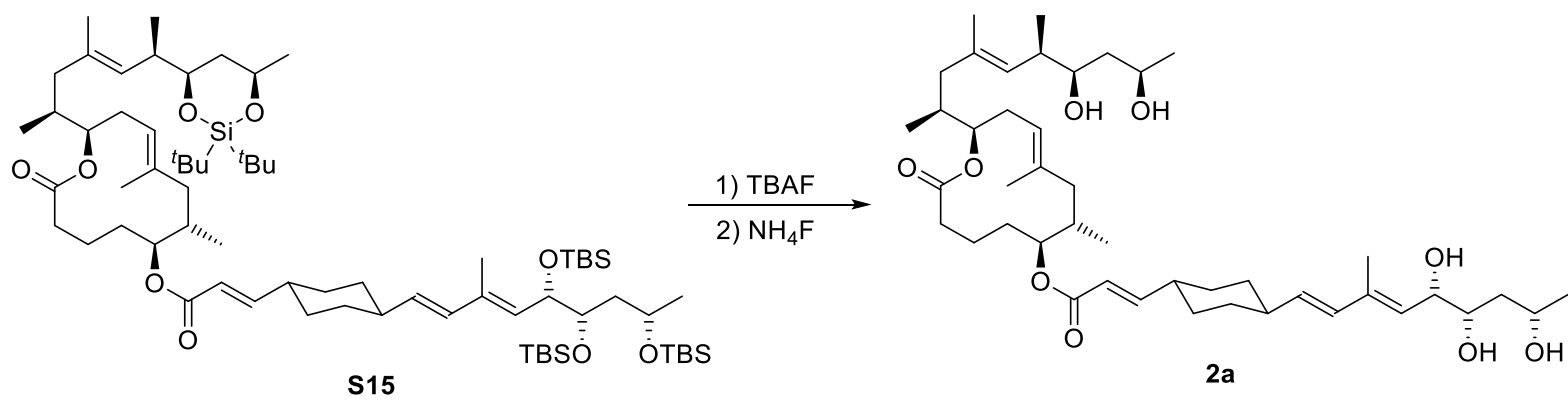

$(E)-(6 S, 7 S, 12 R, E)-12-((2 S, 6 R, 7 R, 9 R, E)-7,9-D i h y d r o x y-4,6-d i m e t h y l d e c-4-e n-2-y l)-7,9-$

dimethyl-2-oxooxacyclododec-9-en-6-yl 3-((1S,4S)-4-((1E,3E,5S,6S,8S)-5,6,8-trihydroxy3-methylnona-1,3-dien-1-yl)cyclohexyl)acrylate (2a). To a solution of protected pentol S15 (30.0 mg, $24.4 \mu \mathrm{mol})$ in THF (2.0 mL) was added TBAF (1 M in THF, $366 \mu \mathrm{L}, 366 \mu \mathrm{mol}$ ) at room temperature and the resulting pale yellow solution was stirred for $2.5 \mathrm{~h}$. $\mathrm{NH}_{4} \mathrm{~F}(45.2 \mathrm{mg}$, $1.22 \mathrm{mmol}$ ) was added and stirring was continued for $2 \mathrm{~d}$ with an additional portion of $\mathrm{NH}_{4} \mathrm{~F}$ (45 mg, $1.22 \mathrm{mmol})$ being added after $24 \mathrm{~h}$. Sat. aq. $\mathrm{NH}_{4} \mathrm{Cl}$ solution $(8 \mathrm{~mL})$ and EtOAc $(15 \mathrm{~mL})$ were added, the layers were separated and the aqueous layer was extracted with EtOAc $(2 \times 15$ $\mathrm{mL})$. The combined organic layers were washed with sat. aq. $\mathrm{NH}_{4} \mathrm{Cl}$ solution $(3 \mathrm{~mL})$, sat. aq. $\mathrm{NaHCO}_{3}$ solution $(3 \mathrm{~mL})$ and brine, dried over $\mathrm{MgSO}_{4}$ and concentrated under reduced pressure. Purification by FC ( $n$-hexane/EtOAc 1:1 $\rightarrow$ EtOAc $\rightarrow$ EtOAc/MeOH 19:1) yielded $14.7 \mathrm{mg}(19.7 \mu \mathrm{mol}, 81 \%)$ of mycolactone analog $\mathbf{2} \mathbf{a}$ as a colorless oil.

$\mathbf{R}_{\mathbf{f}}(\mathrm{EtOAc})=0.15 ;[\alpha]_{\mathbf{D}^{25}}{ }^{25}-35.5^{\circ}\left(\mathrm{c}=0.38, \mathrm{CHCl}_{3}\right) ;{ }^{1} \mathbf{H}-\mathbf{N M R}\left(400 \mathrm{MHz}, \mathrm{CDCl}_{3}\right) \delta 6.91(\mathrm{dd}$, $J=15.7,6.7 \mathrm{~Hz}, 1 \mathrm{H}), 6.04(\mathrm{~d}, J=15.8 \mathrm{~Hz}, 1 \mathrm{H}), 5.76(\mathrm{dd}, J=15.8,1.4 \mathrm{~Hz}, 1 \mathrm{H}), 5.65(\mathrm{dd}, J=$ $15.8,6.9 \mathrm{~Hz}, 1 \mathrm{H}), 5.33(\mathrm{~d}, J=9.0 \mathrm{~Hz}, 1 \mathrm{H}), 5.08(\mathrm{~d}, J=10.5 \mathrm{~Hz}, 1 \mathrm{H}), 5.01-4.85(\mathrm{~m}, 2 \mathrm{H}), 4.79$ - $4.63(\mathrm{~m}, 1 \mathrm{H}), 4.34-4.20(\mathrm{~m}, 1 \mathrm{H}), 4.13-3.94(\mathrm{~m}, 2 \mathrm{H}), 3.77-3.68(\mathrm{~m}, 1 \mathrm{H}), 3.67-3.58(\mathrm{~m}$, $1 \mathrm{H}), 3.53(\mathrm{~s}, 1 \mathrm{H}), 3.18-2.98(\mathrm{~m}, 2 \mathrm{H}), 2.83(\mathrm{~d}, J=4.1 \mathrm{~Hz}, 1 \mathrm{H}), 2.55-2.33(\mathrm{~m}, 3 \mathrm{H}), 2.25$ (s, $1 \mathrm{H}), 2.17-1.35(\mathrm{~m}, 31 \mathrm{H}), 1.28-1.11(\mathrm{~m}, 10 \mathrm{H}), 0.99(\mathrm{~d}, J=6.7 \mathrm{~Hz}, 3 \mathrm{H}), 0.90(\mathrm{~d}, J=6.4 \mathrm{~Hz}$, $3 \mathrm{H}), 0.86(\mathrm{~d}, J=6.7 \mathrm{~Hz}, 3 \mathrm{H}) ;{ }^{13} \mathbf{C}-\mathbf{N M R}\left(100 \mathrm{MHz}, \mathrm{CDCl}_{3}\right) \delta 173.51,166.54,153.93,138.53$, 136.89, 136.19, 134.37, 132.02, 129.18, 128.02, 122.75, 119.42, 78.81, 77.36, 76.09, 72.19, 69.36, 68.66, 45.66, 43.67, 41.95, 40.56, 40.21, 39.39, 35.59, 34.90, 32.37, 32.34, 32.14, 31.40, 31.38, 30.35, 29.55, 24.31, 24.21, 20.33, 20.17, 16.76, 16.29, 15.88, 14.77, 13.47; IR (film, EtOAc): $\widetilde{v}=3379,2962,2927,2873,1724,1452,1378,1253,1215,1162,1131,1090,1040$, 1014, 983; HR-MS (ESI) calc. 767.5069 for $\mathrm{C}_{44} \mathrm{H}_{72} \mathrm{NaO}_{9}{ }^{+}[\mathrm{M}+\mathrm{Na}]^{+}$, found 767.5059. 

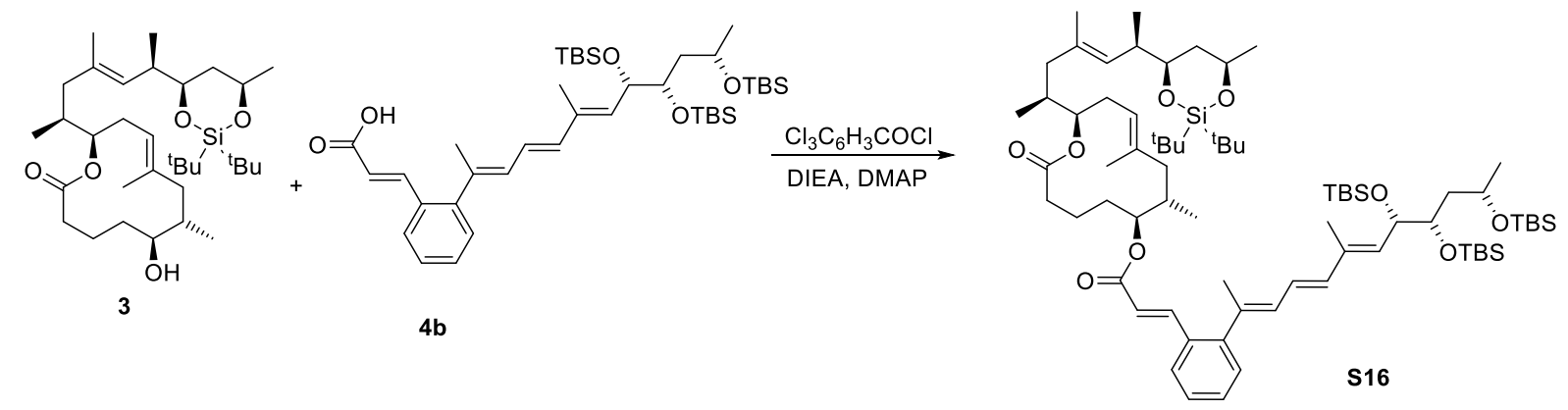

$(2 E, 8 E, 10 E, 12 S, 13 S, 15 S)-(6 S, 7 S, 12 R, E)-12-((2 S, 6 R, E)-6-((4 R, 6 S)-6-(A c e t o x y m e t h y l)-2,2-$ di-tert-butyl-1,3,2-dioxasilinan-4-yl)-4-methylhept-4-en-2-yl)-7,9-dimethyl-2-oxooxacyclododec-9-en-6-yl 12,13,15-tris((tert-butyldimethylsilyl)oxy)-4,6,10-trimethylhexadeca-2,4,6,8,10-pentaenoate (S16). To a solution of acid $\mathbf{4 b}(19.4 \mathrm{mg}, 27.1 \mu \mathrm{mol})$ and DMAP $(11.6 \mathrm{mg}, 94.9 \mu \mathrm{mol})$ in THF $(1.5 \mathrm{~mL})$ were added DIEA $(18.5 \mu \mathrm{L}, 109 \mu \mathrm{mol})$ and 2,4,6trichlorobenzoyl chloride $(8.46 \mu \mathrm{L}, 54.2 \mu \mathrm{mol})$ at room temperature. After a few seconds the solution became turbid. A solution of alcohol $3^{2}(7.68 \mathrm{mg}, 13.6 \mu \mathrm{mol})$ in THF $(1.0 \mathrm{~mL}+0.5$ $\mathrm{mL}$ for rinsing) was added and the yellow suspension was protected from light and stirred for $4 \mathrm{~h}$ at room temperature over which period it turned white. The reaction was quenched by adding sat. aq. $\mathrm{NaHCO}_{3}$ solution $(10 \mathrm{~mL})$ and EtOAc $(10 \mathrm{~mL})$. The layers were separated and the aqueous layer was extracted with EtOAc $(4 \times 10 \mathrm{~mL})$. The combined organic layers were washed with brine, dried over $\mathrm{MgSO}_{4}$ and concentrated under reduced pressure. Purification by FC ( $n$-hexane/EtOAc 25:1 $\rightarrow n$-hexane/EtOAc 1:1+1\% HOAc) yielded $20.2 \mathrm{mg}$ of the impure ester as a colorless oil and $6.2 \mathrm{mg}(8.7 \mu \mathrm{mol}, 64 \%)$ of the starting acid $\mathbf{4 b}$. A second column ( $n$-hexane/EtOAc 50:1) furnished $16.6 \mathrm{mg}$ (13.2 $\mu \mathrm{mol}$, 97\%) of ester $\mathbf{S 1 6}$ as a colorless oil.

$\boldsymbol{R}_{\boldsymbol{f}}\left(n\right.$-hexane/EtOAc 25:1) $=0.22 ;[\boldsymbol{\alpha}] \mathbf{D}^{\mathbf{2 0}}=-24.50^{\circ}\left(\mathrm{c}=1.00, \mathrm{CHCl}_{3}\right) ;{ }^{\mathbf{1}} \mathbf{H}-\mathbf{N M R}(400 \mathrm{MHz}$, $\left.\mathrm{CDCl}_{3}\right) \delta 7.81(\mathrm{~d}, J=16.0 \mathrm{~Hz}, 1 \mathrm{H}), 7.59(\mathrm{dd}, J=7.8,1.4 \mathrm{~Hz}, 1 \mathrm{H}), 7.33(\mathrm{td}, J=7.4,1.4 \mathrm{~Hz}$, 1H), $7.29-7.25(\mathrm{~m}, 1 \mathrm{H}), 7.25-7.20(\mathrm{~m}, 1 \mathrm{H}), 6.53(\mathrm{dd}, J=15.3,11.0 \mathrm{~Hz}, 1 \mathrm{H}), 6.36(\mathrm{~d}, J=$ $15.9 \mathrm{~Hz}, 1 \mathrm{H}), 6.28(\mathrm{~d}, J=15.2 \mathrm{~Hz}, 1 \mathrm{H}), 6.04(\mathrm{dd}, J=11.0,1.7 \mathrm{~Hz}, 1 \mathrm{H}), 5.53(\mathrm{~d}, J=9.1 \mathrm{~Hz}$, $1 \mathrm{H}), 5.07(\mathrm{~d}, J=10.4 \mathrm{~Hz}, 1 \mathrm{H}), 4.95(\mathrm{~d}, J=9.6 \mathrm{~Hz}, 1 \mathrm{H}), 4.88(\mathrm{ddd}, J=11.8,5.8,2.9 \mathrm{~Hz}, 1 \mathrm{H})$, $4.81-4.72(\mathrm{~m}, 1 \mathrm{H}), 4.44(\mathrm{dd}, J=9.2,3.6 \mathrm{~Hz}, 1 \mathrm{H}), 4.20-4.06(\mathrm{~m}, 1 \mathrm{H}), 3.99-3.84(\mathrm{~m}, 1 \mathrm{H})$, $3.74-3.66(\mathrm{~m}, 1 \mathrm{H}), 3.64(\mathrm{dd}, J=8.1,4.2 \mathrm{~Hz}, 1 \mathrm{H}), 2.50-2.36(\mathrm{~m}, 2 \mathrm{H}), 2.35-2.24(\mathrm{~m}, 1 \mathrm{H})$, $2.14(\mathrm{~d}, J=1.3 \mathrm{~Hz}, 3 \mathrm{H}), 2.13-2.01(\mathrm{~m}, 4 \mathrm{H}), 2.01-1.90(\mathrm{~m}, 3 \mathrm{H}), 1.88(\mathrm{~d}, J=1.2 \mathrm{~Hz}, 3 \mathrm{H})$, $1.87-1.74(\mathrm{~m}, 3 \mathrm{H}), 1.74-1.66(\mathrm{~m}, 1 \mathrm{H}), 1.68(\mathrm{~s}, 3 \mathrm{H}), 1.66-1.56(\mathrm{~m}, 3 \mathrm{H}), 1.60(\mathrm{~d}, J=1.3 \mathrm{~Hz}$, $3 \mathrm{H}), 1.40-1.29(\mathrm{~m}, 2 \mathrm{H}), 1.28(\mathrm{~s}, 3 \mathrm{H}), 1.18(\mathrm{~d}, J=6.0 \mathrm{~Hz}, 3 \mathrm{H}), 1.14(\mathrm{~d}, J=6.0 \mathrm{~Hz}, 3 \mathrm{H}), 1.00$ (s, 9H), $1.00-0.98(\mathrm{~m}, 3 \mathrm{H}), 0.98(\mathrm{~s}, 9 \mathrm{H}), 0.93$ (d, J = 5.6 Hz, 3H), $0.88(\mathrm{~s}, 18 \mathrm{H}), 0.86(\mathrm{~s}, 9 \mathrm{H})$, 
0.06 (s, 3H), 0.05 (s, 3H), 0.05 (s, 3H), 0.04 (s, 3H), 0.03 (s, 3H), -0.02 (s, 3H); ${ }^{13}$ C-NMR $\left(101 \mathrm{MHz} \mathrm{CDCl}_{3}\right) \delta 173.32,166.42,146.58,143.86,138.81,136.83,135.94,134.64,133.48$, 132.85, 132.27, 131.97, 129.99, 129.87, 128.89, 127.21, 126.86, 123.89, 122.74, 119.09, 79.21, 78.01, 76.13, 73.80, 71.48, 70.68, 66.23, 45.71, 43.56, 42.87, 41.86, 40.57, 35.61, 34.91, 32.04, $30.50,29.84,29.71,27.73,27.34,26.08,25.98,25.08,23.66,22.85,20.52,20.09,19.72$, 19.52, $18.30,18.23,18.12,16.84,16.22,15.90,14.62,13.67,-3.90,-3.99,-4.15,-4.34,-4.43,-4.52$; IR (film): $\tilde{v}=2957,2928,2894,2856,1729,1714,1631,1472,1460,1377,1360,1309,1250$, 1159, 1132, 1097, 1064, 1003, 981, 962, 938, 912, 886, 834, 807, 773, 650; HR-MS (ESI): m/z calc. for $\mathrm{C}_{73} \mathrm{H}_{132} \mathrm{NO}_{9} \mathrm{Si}_{4}\left[\mathrm{M}+\mathrm{NH}_{4}\right]^{+}:$1278.8974, found: 1278.8964 .
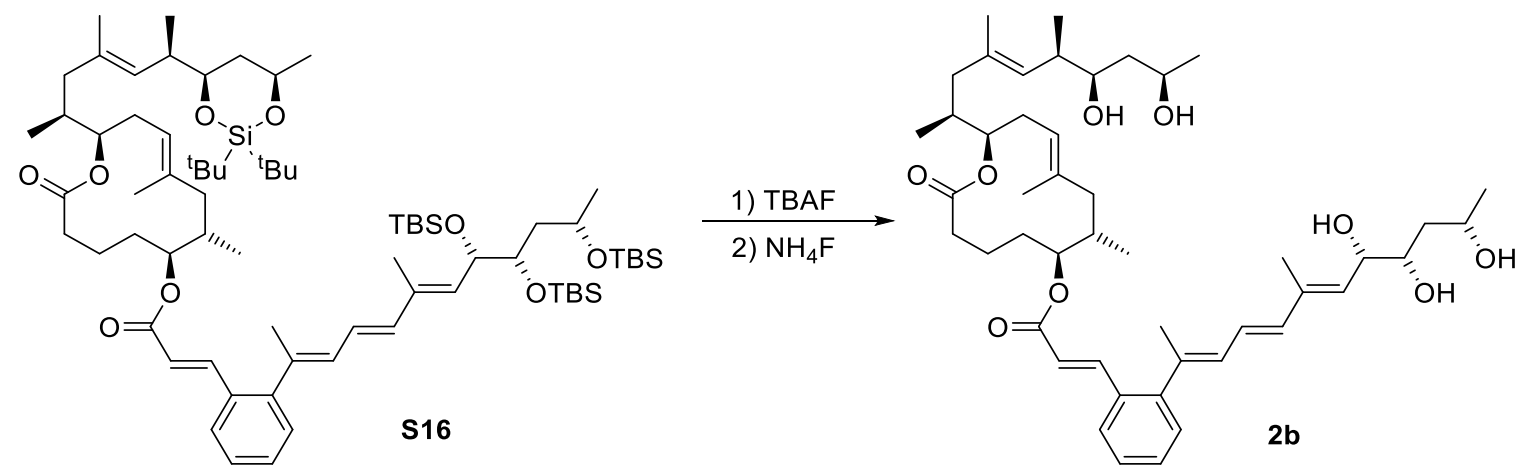

$(6 S, 7 S, 12 R, E)-12-((2 S, 6 R, 7 R, 9 R, E)-7,9-D i h y d r o x y-4,6-d i m e t h y l d e c-4-e n-2-y l)-7,9-d i-$

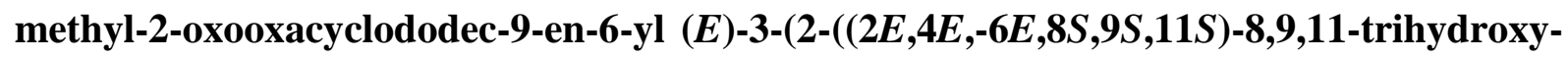
6-methyldodeca-2,4,6-trien-2-yl)phenyl)acrylate (2b). To a solution of the protected pentol S16 $(10.0 \mathrm{mg}, 7.92 \mu \mathrm{mol})$ in THF (4.0 mL) was added TBAF (1 M in THF, $198 \mu \mathrm{L}, 198 \mu \mathrm{mol})$ at room temperature. The solution was stirred for $2.5 \mathrm{~h}$ at room temperature, at which point all TBS groups were removed according to LC-MS analysis. $\mathrm{NH}_{4} \mathrm{~F}(29.3 \mathrm{mg}, 792 \mu \mathrm{mol})$ was added and the solution was stirred for another $16 \mathrm{~h}$ before the reaction mixture was directly loaded onto a Pasteur pipette filled with silica and eluted with THF followed by THF/MeOH 19:1. After solvent evaporation, the product was purified by $\mathrm{FC}\left(\mathrm{CH}_{2} \mathrm{Cl}_{2} / \mathrm{MeOH} 25: 1\right)$ to obtain the mycolactone analog $\mathbf{2 b}(2.3 \mathrm{mg}, 3.0 \mu \mathrm{mol}, 37 \%)^{*}$ as a colorless oil.

*Only the purest fractions were included for yield determination. Conversion was relatively clean and additional $4.2 \mathrm{mg}$ of a contaminated product fraction (mainly with TBAF but also minor isomers) were obtained.

$\mathbf{R}_{\mathbf{f}}=0.32\left(\mathrm{CH}_{2} \mathrm{Cl}_{2} / \mathrm{MeOH} 9: 1\right) ;[\boldsymbol{\alpha}]_{\mathbf{D}^{\mathbf{2 0}}}=-18.00^{\circ}\left(\mathrm{c}=0.20, \mathrm{CHCl}_{3}\right) ;{ }^{1} \mathbf{H}-\mathbf{N M R}(500 \mathrm{MHz}$, Acetone- $\left.d_{6}\right) \delta 7.85(\mathrm{~d}, J=16.0 \mathrm{~Hz}, 1 \mathrm{H}), 7.78(\mathrm{~d}, J=7.8 \mathrm{~Hz}, 1 \mathrm{H}), 7.43(\mathrm{td}, J=7.5,1.3 \mathrm{~Hz}, 1 \mathrm{H})$, $7.39-7.31(\mathrm{~m}, 2 \mathrm{H}), 6.72(\mathrm{dd}, J=15.2,11.0 \mathrm{~Hz}, 1 \mathrm{H}), 6.47(\mathrm{~d}, J=15.9 \mathrm{~Hz}, 1 \mathrm{H}), 6.38(\mathrm{~d}, J=$ $15.2 \mathrm{~Hz}, 1 \mathrm{H}), 6.02(\mathrm{dd}, J=11.0,1.6 \mathrm{~Hz}, 1 \mathrm{H}), 5.58(\mathrm{~d}, J=8.8 \mathrm{~Hz}, 1 \mathrm{H}), 5.17-5.10(\mathrm{~m}, 1 \mathrm{H})$, 
$5.05(\mathrm{~d}, J=9.6 \mathrm{~Hz}, 1 \mathrm{H}), 4.91(\mathrm{ddd}, J=11.8,5.2,2.7 \mathrm{~Hz}, 1 \mathrm{H}), 4.34-4.23(\mathrm{~m}, 3 \mathrm{H}), 4.05-3.88$ (m, 2H), $3.74-3.66(\mathrm{~m}, 1 \mathrm{H}), 3.56-3.48(\mathrm{~m}, 1 \mathrm{H}), 2.51(\mathrm{dt}, J=14.0,11.5 \mathrm{~Hz}, 1 \mathrm{H}), 2.45-2.36$ $(\mathrm{m}, 2 \mathrm{H}), 2.20(\mathrm{~d}, J=0.9 \mathrm{~Hz}, 3 \mathrm{H}), 2.14(\mathrm{dd}, J=13.2,6.6 \mathrm{~Hz}, 1 \mathrm{H}), 1.98(\mathrm{~s}, 5 \mathrm{H}), 1.96(\mathrm{~d}, J=1.1$ $\mathrm{Hz}, 3 \mathrm{H}), 1.85(\mathrm{dd}, J=13.1,8.5 \mathrm{~Hz}, 1 \mathrm{H}), 1.78-1.65(\mathrm{~m}, 3 \mathrm{H}), 1.73(\mathrm{~s}, 3 \mathrm{H}), 1.66$ (d, $J=1.3 \mathrm{~Hz}$, $3 \mathrm{H}), 1.65-1.48(\mathrm{~m}, 4 \mathrm{H}), 1.45-1.36(\mathrm{~m}, 1 \mathrm{H}), 1.16(\mathrm{~d}, J=6.0 \mathrm{~Hz}, 3 \mathrm{H}), 1.14(\mathrm{~d}, J=6.1 \mathrm{~Hz}$, $3 \mathrm{H}), 0.99(\mathrm{~d}, J=6.6 \mathrm{~Hz}, 3 \mathrm{H}), 0.93(\mathrm{~d}, J=6.4 \mathrm{~Hz}, 3 \mathrm{H}), 0.90(\mathrm{~d}, J=6.8 \mathrm{~Hz}, 3 \mathrm{H}) ;{ }^{13} \mathbf{C}-\mathbf{N M R}(126$ MHz, Acetone- $\left.d_{6}\right) \delta 172.48,165.65,146.09,143.25,138.45,136.44,136.29,135.61,133.22$, 132.58, 132.30, 131.93, 130.33, 129.83, 128.47, 127.28, 126.91, 124.11, 122.98, 118.86, 78.63, 76.08 (major), 75.97 (minor), 75.47, 74.91 (major), 74.80 (minor), 71.49 (major), 71.39 (minor), 68.10 (major), 67.98 (minor), 66.86 (major), 66.75 (minor), 45.54, 43.50, 42.91, 40.98, 39.60, 35.16, 34.47, 31.97, 30.68, 23.73, 23.37, 19.91, 19.66, 18.39, 16.29, 15.36, 15.08, 14.12, 12.60; IR (film): $\tilde{v}=3300,2960,2926,2873,2856,1715,1627,1458,1378,1309,1252,1159$, 1130, 1013, 987, 964, 883, 756; HR-MS (ESI): $m / z$ calc. for $\mathrm{C}_{47} \mathrm{H}_{70} \mathrm{NaO} 9[\mathrm{M}+\mathrm{Na}]^{+}: 801.4912$, found: 801.4901 .

Synthesis of mycolactone analog $\mathbf{2 c}$
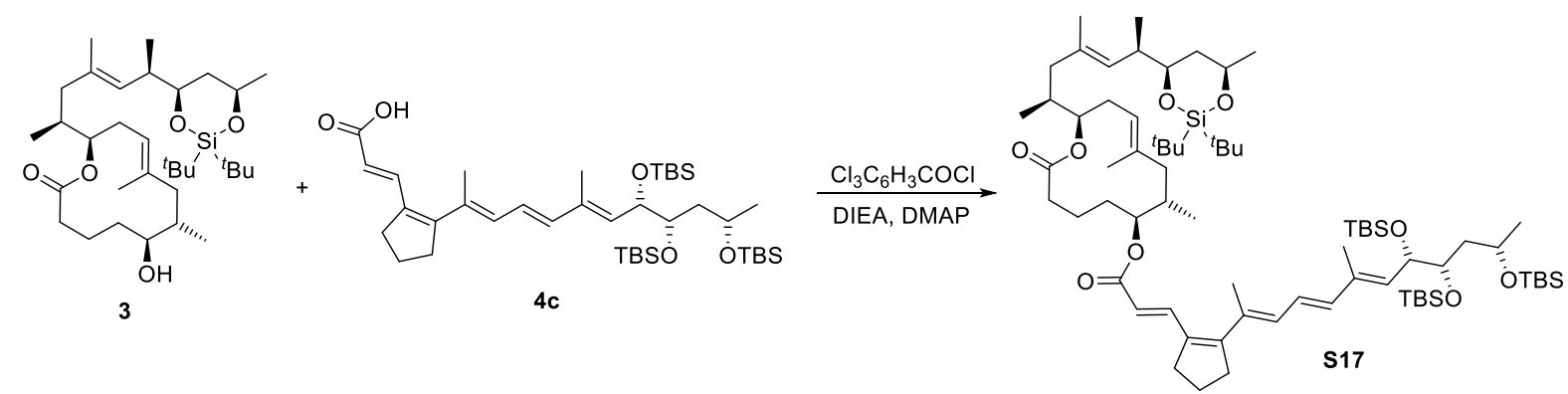

$(2 E, 8 E, 10 E, 12 S, 13 S, 15 S)-(6 S, 7 S, 12 R, E)-12-((2 S, 6 R, E)-6-((4 R, 6 S)-6-(A c e t o x y m e t h y l)-2,2-$ di-tert-butyl-1,3,2-dioxasilinan-4-yl)-4-methylhept-4-en-2-yl)-7,9-dimethyl-2-oxooxacyclododec-9-en-6-yl 12,13,15-tris((tert-butyldimethylsilyl)oxy)-4,6,10-trimethylhexadeca-2,4,6,8,10-pentaenoate (S17). To a solution of acid $\mathbf{4 c}(42.0 \mathrm{mg}, 58.7 \mu \mathrm{mol})$ and DMAP (25.1 mg, $206 \mu \mathrm{mol})$ in THF $(2 \mathrm{~mL})$ were added DIEA $(40.1 \mu \mathrm{L}, 235 \mu \mathrm{mol})$ and 2,4,6trichlorobenzoyl chloride $(18.4 \mu \mathrm{L}, 117 \mu \mathrm{mol})$ at room temperature. After a few seconds the solution became turbid. A solution of alcohol $3^{2}(16.6 \mathrm{mg}, 29.4 \mu \mathrm{mol})$ in THF $(1.0 \mathrm{~mL}+0.5$ $\mathrm{mL}$ for rinsing) was added. The yellow suspension was protected from light and stirred for $2 \mathrm{~h}$ at room temperature. The reaction was quenched by adding sat. aq. $\mathrm{NaHCO}_{3}$ solution $(20 \mathrm{~mL})$ and EtOAc $(30 \mathrm{~mL})$, the layers were separated and the aqueous layer was extracted with EtOAc (3 x $30 \mathrm{~mL}$ ). The combined organic layers were washed with brine, dried over $\mathrm{MgSO}_{4}$ and 
concentrated under reduced pressure. Purification by FC (n-hexane/EtOAc 70:1 $\rightarrow n$ hexane/EtOAc 50:1 $\rightarrow n$-hexane/EtOAc 6:1, $1 \% \mathrm{MeOH}, 0.01 \%$ HOAc) yielded $34.1 \mathrm{mg}$ (27.2 $\mu \mathrm{mol}, 93 \%)$ of ester $\mathbf{S 1 7}$ as a yellow oil and $16.9 \mathrm{mg}(24.0 \mu \mathrm{mol}, 82 \%)$ of recovered starting acid $4 c$.

$\boldsymbol{R}_{\boldsymbol{f}}\left(n\right.$-hexane/EtOAc 20:1) $=0.26 ;[\boldsymbol{\alpha}] \mathbf{D}^{\mathbf{2 0}}=-20.90^{\circ}\left(\mathrm{c}=1.00, \mathrm{CHCl}_{3}\right) ;{ }^{\mathbf{1}} \mathbf{H}-\mathbf{N M R}(400 \mathrm{MHz}$, $\left.\mathrm{CDCl}_{3}\right) \delta 7.79(\mathrm{~d}, J=15.5 \mathrm{~Hz}, 1 \mathrm{H}), 6.50(\mathrm{dd}, J=15.1,11.0 \mathrm{~Hz}, 1 \mathrm{H}), 6.33(\mathrm{~d}, J=15.2 \mathrm{~Hz}, 1 \mathrm{H})$, $6.17(\mathrm{~d}, J=10.9 \mathrm{~Hz}, 1 \mathrm{H}), 5.76(\mathrm{~d}, J=15.5 \mathrm{~Hz}, 1 \mathrm{H}), 5.55(\mathrm{~d}, J=9.1 \mathrm{~Hz}, 1 \mathrm{H}), 5.05(\mathrm{~d}, J=10.6$ $\mathrm{Hz}, 1 \mathrm{H}), 4.95(\mathrm{~d}, J=9.7 \mathrm{~Hz}, 1 \mathrm{H}), 4.87(\mathrm{ddd}, J=12.0,5.7,2.9 \mathrm{~Hz}, 1 \mathrm{H}), 4.78-4.68(\mathrm{~m}, 1 \mathrm{H})$, $4.43(\mathrm{dd}, J=9.2,3.6 \mathrm{~Hz}, 1 \mathrm{H}), 4.21-4.07(\mathrm{~m}, 1 \mathrm{H}), 3.97-3.85(\mathrm{~m}, 1 \mathrm{H}), 3.69(\mathrm{ddd}, J=11.2$, 7.5, $1.8 \mathrm{~Hz}, 1 \mathrm{H}), 3.63(\mathrm{dt}, J=7.9,3.9 \mathrm{~Hz}, 1 \mathrm{H}), 2.67(\mathrm{q}, J=7.2 \mathrm{~Hz}, 2 \mathrm{H}), 2.59$ (t, $J=7.9 \mathrm{~Hz}$, $2 \mathrm{H}), 2.48-2.26(\mathrm{~m}, 3 \mathrm{H}), 2.12-2.03(\mathrm{~m}, 3 \mathrm{H}), 2.01(\mathrm{~d}, J=1.2 \mathrm{~Hz}, 3 \mathrm{H}), 1.99-1.86(\mathrm{~m}, 5 \mathrm{H})$, $1.85(\mathrm{~d}, J=1.3 \mathrm{~Hz}, 3 \mathrm{H}), 1.84-1.70(\mathrm{~m}, 4 \mathrm{H}), 1.67(\mathrm{~s}, 3 \mathrm{H}), 1.66-1.61(\mathrm{~m}, 3 \mathrm{H}), 1.60$ (d, $J=1.3$ $\mathrm{Hz}, 3 \mathrm{H}), 1.59-1.50(\mathrm{~m}, 1 \mathrm{H}), 1.39-1.29(\mathrm{~m}, 2 \mathrm{H}), 1.18(\mathrm{~d}, J=6.1 \mathrm{~Hz}, 3 \mathrm{H}), 1.14(\mathrm{~d}, J=6.0$ $\mathrm{Hz}, 3 \mathrm{H}), 1.00$ (s, 9H), 0.99 (d, J=6.2 Hz, 3H), 0.97 (s, 9H), 0.92 (d, J=5.9 Hz, 3H), 0.88 (s, 9H), 0.87 (s, 9H), $0.87-0.86(\mathrm{~m}, 9 \mathrm{H}), 0.84(\mathrm{~d}, J=6.4 \mathrm{~Hz}, 3 \mathrm{H}), 0.05(\mathrm{~d}, J=1.2 \mathrm{~Hz}, 9 \mathrm{H}), 0.03$ (s, 3H), 0.02 (s, 3H), -0.02 (s, 3H); ${ }^{13} \mathbf{C}-\mathbf{N M R}\left(101 \mathrm{MHz}, \mathrm{CDCl}_{3}\right) \delta$ 173.34, 167.23, 153.46, $140.06,139.43,136.88,134.81,134.37,133.74,133.32,132.86,131.59,129.97,123.81$, 122.66, 118.33, 78.85, 78.01, 76.12, 73.80, 71.50, 70.68, 66.22, 45.71, 43.56, 42.84, 41.86, $40.58,37.45,35.63,34.90,33.74,32.03,30.51,29.71,27.73,27.35,26.09,26.06,25.98,25.08$, 23.64, 22.86, 21.84, 20.54, 20.05, 19.73, 18.31, 18.23, 18.11, 16.92, 16.84, 16.21, 15.89, 14.62, 13.69, -3.91, -3.99, -4.17, -4.35, -4.42, -4.54; IR (film): $\tilde{v}=2957,2930,2891,2857,1730$, 1707, 1608, 1472, 1462, 1376, 1361, 1299, 1249, 1154, 1098, 1064, 1005, 981, 961, 939, 913, 885, 835, 775, 651; HR-MS (ESI): $m / z$ calc. for $\mathrm{C}_{72} \mathrm{H}_{130} \mathrm{NaO}_{9} \mathrm{Si}_{4}[\mathrm{M}+\mathrm{Na}]^{+}: 1273.8684$, found: 1273.8682 . 

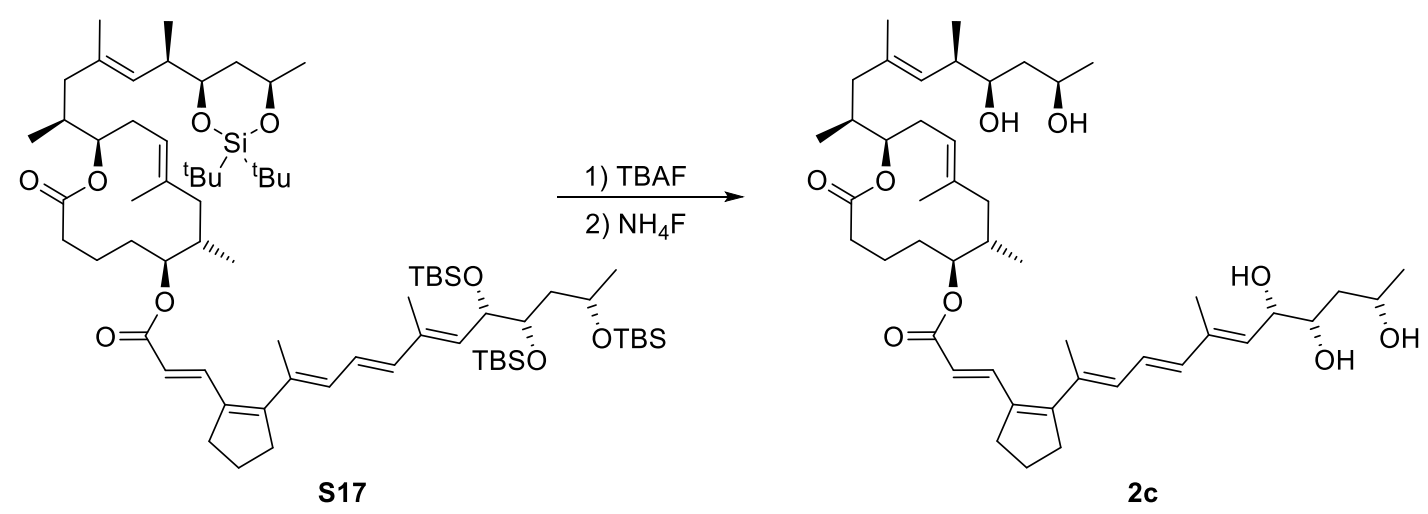

$(6 S, 7 S, 12 R, E)-12-((2 S, 6 R, 7 R, 9 R, E)-7,9-D i h y d r o x y-4,6-d i m e t h y l d e c-4-e n-2-y l)-7,9-d i-$ methyl-2-oxooxacyclododec-9-en-6-yl $(E)-3-(2-((2 E, 4 E, 6 E, 8 S, 9 S, 11 S)-8,9,11-t r i h y d r o x y-$ 6-methyldodeca-2,4,6-trien-2-yl)cyclopent-1-en-1-yl)acrylate (2c). To a solution of protected pentol S17 (10.0 mg, $7.99 \mu \mathrm{mol})$ in THF (4 mL) was added TBAF (1 M in THF, 200 $\mu \mathrm{L}, 200 \mu \mathrm{mol})$ at room temperature. The solution was protected from light and stirred for $2.5 \mathrm{~h}$ at which point all TBS groups were removed according to LC-MS analysis. $\mathrm{NH}_{4} \mathrm{~F}(29.6 \mathrm{mg}$, $799 \mu \mathrm{mol})$ was added and stirring was continued for another $15 \mathrm{~h}$ at room temperature. The reaction mixture was directly loaded onto a Pasteur pipette filled with silica and eluted with THF, followed by THF/MeOH 19:1. After solvent evaporation, the product was purified twice by $\mathrm{FC}\left(\mathrm{CH}_{2} \mathrm{Cl}_{2} / \mathrm{MeOH} 25: 1\right)$ to obtain the mycolactone analog $2 \mathrm{c}(3.1 \mathrm{mg}, 4.0 \mu \mathrm{mol}, 50 \%)^{*}$ as a yellow oil.

*Only the purest fractions were included for yield determination. Conversion was relatively clean and additional $4.7 \mathrm{mg}$ of a strongly contaminated fraction (mainly with TBAF and some minor isomers) were obtained.

${ }^{1} \mathbf{H}-N M R\left(400 \mathrm{MHz}\right.$, Acetone- $\left.d_{6}\right) \delta 7.75(\mathrm{~d}, J=15.6 \mathrm{~Hz}, 1 \mathrm{H}), 6.66(\mathrm{dd}, J=15.1,11.0 \mathrm{~Hz}, 1 \mathrm{H})$, $6.41(\mathrm{~d}, J=15.2 \mathrm{~Hz}, 1 \mathrm{H}), 6.18(\mathrm{~d}, J=11.0 \mathrm{~Hz}, 1 \mathrm{H}), 5.79(\mathrm{~d}, J=15.6 \mathrm{~Hz}, 1 \mathrm{H}), 5.58(\mathrm{~d}, J=8.9$ $\mathrm{Hz}, 1 \mathrm{H}), 5.11(\mathrm{~d}, J=11.1 \mathrm{~Hz}, 1 \mathrm{H}), 5.04(\mathrm{~d}, J=9.6 \mathrm{~Hz}, 1 \mathrm{H}), 4.88$ (ddd, $J=11.8,5.2,2.8 \mathrm{~Hz}$, $1 \mathrm{H}), 4.69(\mathrm{dt}, J=8.7,4.1 \mathrm{~Hz}, 1 \mathrm{H}), 4.33-4.25(\mathrm{~m}, 1 \mathrm{H}), 4.02-3.93(\mathrm{~m}, 2 \mathrm{H}), 3.68(\mathrm{ddt}, J=9.7$, 6.6, $3.3 \mathrm{~Hz}, 1 \mathrm{H}), 3.53-3.46(\mathrm{~m}, 1 \mathrm{H}), 2.70(\mathrm{t}, J=7.6 \mathrm{~Hz}, 2 \mathrm{H}), 2.62(\mathrm{t}, J=7.6 \mathrm{~Hz}, 2 \mathrm{H}), 2.49$ $(\mathrm{dt}, J=13.9,11.5 \mathrm{~Hz}, 1 \mathrm{H}), 2.44-2.34(\mathrm{~m}, 2 \mathrm{H}), 2.12(\mathrm{dd}, J=12.9,6.6 \mathrm{~Hz}, 1 \mathrm{H}), 2.04-1.86$ $(\mathrm{m}, 8 \mathrm{H}), 2.01(\mathrm{~d}, J=1.2 \mathrm{~Hz}, 3 \mathrm{H}), 1.92(\mathrm{~d}, J=1.2 \mathrm{~Hz}, 3 \mathrm{H}), 1.83(\mathrm{dd}, J=13.1,8.4 \mathrm{~Hz}, 1 \mathrm{H}), 1.70$ (s, 3H), $1.76-1.62(\mathrm{~m}, 2 \mathrm{H}), 1.65(\mathrm{~d}, J=1.2 \mathrm{~Hz}, 3 \mathrm{H}), 1.62-1.45(\mathrm{~m}, 4 \mathrm{H}), 1.45-1.32(\mathrm{~m}, 2 \mathrm{H})$, $1.14(\mathrm{~d}, J=5.9 \mathrm{~Hz}, 3 \mathrm{H}), 1.12(\mathrm{~d}, J=5.4 \mathrm{~Hz}, 3 \mathrm{H}), 0.98(\mathrm{~d}, J=6.7 \mathrm{~Hz}, 3 \mathrm{H}), 0.90$ (d, $J=6.4 \mathrm{~Hz}$, 3H), $0.89(\mathrm{~d}, J=4.1 \mathrm{~Hz}, 3 \mathrm{H}) ;{ }^{13} \mathbf{C}-\mathbf{N M R}\left(126 \mathrm{MHz}\right.$, Acetone- $\left.d_{6}\right) \delta 173.22,167.02,154.15$, $140.11,139.71,137.22,137.14,134.88,134.29,134.00,133.36,132.30,131.11,124.85$, $123.71,119.00,79.08,76.85,76.23,75.70,72.31,68.87,67.63,46.34,44.29,43.67,41.75$, 
$40.38,37.57,35.95,35.26,33.91,32.70,31.45,24.52,24.17,22.31,20.64,20.50,17.12$, 16.57 , 16.17, 15.92, 14.94, 13.41. IR (film): $\tilde{v}=3391,2960,2927,1725,1703,1604,1455,1376$, 1302, 1248, 1213, 1157, 1077, 1014, 979, 930, 837; HR-MS (ESI): $m / z$ calc. for $\mathrm{C}_{46} \mathrm{H}_{72} \mathrm{NaO}_{9}$ $[\mathrm{M}+\mathrm{Na}]^{+}:$791.5069, found: 791.5051 . 


\subsection{Optimization of the Stille coupling between stannane 14 and vinyl iodide 12}

Table S1. Conditions tested for the Stille coupling of stannane 14 with vinyl iodide 12.

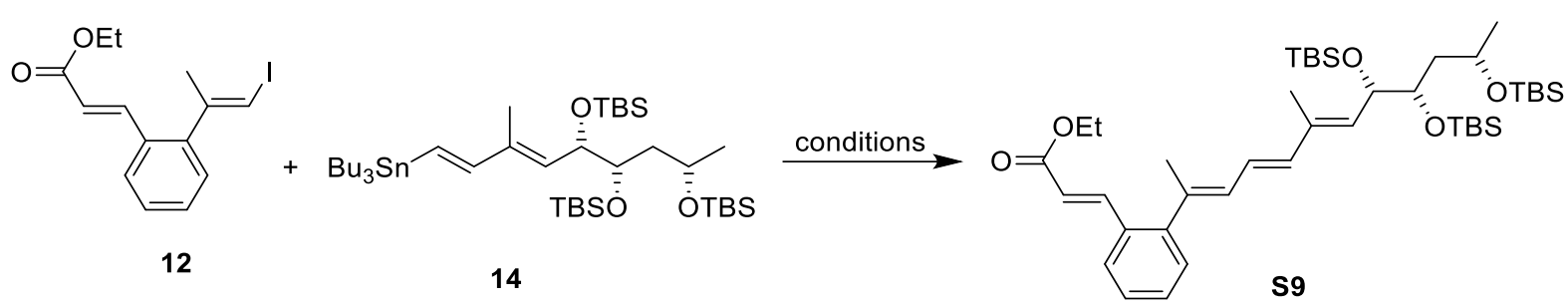

\begin{tabular}{|c|c|c|c|c|c|}
\hline Entry $^{\mathbf{a}}$ & $\begin{array}{c}\text { Catalyst system/ } \\
\text { additives }\end{array}$ & Solvent $^{b}$ & $\mathbf{T}\left[{ }^{\circ} \mathbf{C}\right]$ & $\begin{array}{l}\text { Time } \\
{[\text { h] }}\end{array}$ & Comment \\
\hline 1 & $\mathrm{Pd}\left(\mathrm{CH}_{3} \mathrm{CN}\right)_{2} \mathrm{Cl}_{2}(0.1$ eq $)$ & $\mathrm{DMF}$ & $\mathrm{RT}$ & 1 & Poor conversion \\
\hline 2 & $\begin{array}{l}\mathrm{Pd}\left(\mathrm{CH}_{3} \mathrm{CN}\right)_{2} \mathrm{Cl}_{2} \\
(0.1 \text { eq }+0.2 \text { eq })\end{array}$ & NMP & $\begin{array}{c}\mathrm{RT} \\
\text { then } 45^{\circ} \mathrm{C}\end{array}$ & $\begin{array}{l}4 \\
4\end{array}$ & $\begin{array}{l}\text { Conversion } 40-60 \%{ }^{\mathrm{d}} \\
\text { byproduct formation }\end{array}$ \\
\hline 3 & $\begin{array}{c}\mathrm{Pd}\left(\mathrm{CH}_{3} \mathrm{CN}\right)_{2} \mathrm{Cl}_{2}(0.1 \mathrm{eq}) \\
\mathrm{CuTC}^{\mathrm{c}}(2 \mathrm{eq})\end{array}$ & $\mathrm{DMF}$ & $\mathrm{RT}$ & 24 & Poor conversion \\
\hline 4 & $\mathrm{CuTC}^{\mathrm{c}}(2 \mathrm{eq})$ & NMP & $\begin{array}{c}0^{\circ} \mathrm{C} \text { to } \mathrm{RT}, \\
3 \mathrm{~h}\end{array}$ & 3 & Conversion $10-30 \%$ d,e \\
\hline 5 & $\begin{array}{c}\mathrm{Pd}\left(\mathrm{CH}_{3} \mathrm{CN}\right)_{2} \mathrm{Cl}_{2}(0.1 \mathrm{eq}) \\
\mathrm{TEA}(6 \mathrm{eq})\end{array}$ & $\mathrm{DMF}$ & RT & 3 & Byproduct formation \\
\hline 6 & $\begin{array}{c}\mathrm{Pd}_{2} \mathrm{dba}_{3}(0.05 \mathrm{eq}) \\
\mathrm{AsPh}_{3}(0.4 \mathrm{eq})\end{array}$ & NMP & RT & 30 & Conversion $40-60 \%$ d \\
\hline 7 & $\begin{array}{l}\mathrm{Pd}_{2} \mathrm{dba}_{3}(0.05 \mathrm{eq}) \\
\mathrm{P}(o \text {-furyl })_{3}(0.2 \mathrm{eq})\end{array}$ & NMP & RT & 30 & Conversion $30-50 \%$ d \\
\hline 8 & $\begin{array}{c}\mathrm{Pd}\left(\mathrm{PPh}_{3}\right)_{4}(0.1 \mathrm{eq}) \\
\mathrm{CuI}(1 \mathrm{eq}) \\
\mathrm{CsF}(2 \mathrm{eq})\end{array}$ & DMF & $\mathrm{RT}$ & 3 & $\begin{array}{l}48 \% \\
\text { (isolated yield) }\end{array}$ \\
\hline
\end{tabular}

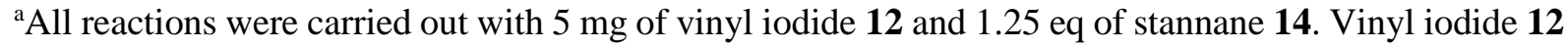
was used for optimization instead of cyclopentene-derived vinyl iodide $\mathbf{1 8}$ due to its higher stability/better accessibility. All reagents/catalysts/additives were combined in a glovebox, the vial was sealed with a septum cap and the solvent $(0.5 \mathrm{~mL}$ or $1 \mathrm{~mL})$ added via cannula. ${ }^{b}$ Dry and degassed solvents were used. ${ }^{\mathrm{c}}$ Copper(I) thiophene-2-carboxylate. ${ }^{\mathrm{d}}$ estimated by TLC. ${ }^{\mathrm{e}}$ conversion did not progress any further after $20 \mathrm{~min}$. 


\section{H/D-exchange in compounds $2 \mathbf{b}$ and $2 \mathbf{c}$ in acetone- $\mathrm{d}_{6}$}

Examination of the ${ }^{13} \mathrm{C}-\mathrm{NMR}$ spectra of mycolactone analogs $\mathbf{2 b}$ and $\mathbf{2 c}$ in acetone- $\mathrm{d}_{6}$ revealed additional sets of upfield-shifted signals for a limited number of peaks, while certain other peaks were broadened. Peak assignment by 2D correlation spectra showed that the affected peaks belong to the carbon atoms attached to the free hydroxy groups at both, the upper core extension $(\mathrm{C} 17, \mathrm{C} 19)$ and the lower side chain $\left(\mathrm{C} 12^{\prime}, \mathrm{C} 13^{\prime}, \mathrm{C} 15^{\prime}\right)$. In the case of compound $\mathbf{2 b}$ (Figure S4), these peaks were properly separated (and thus are labelled as "major" and "minor" in the corresponding NMR peak listings). In the case of 2c, however (Figure S5), most of the affected peaks only had a "shoulder" and were not fully separable (and thus are not highlighted in the corresponding spectral data). Moreover, peak broadening was observed for the signals belonging to the carbon atoms between two hydroxylated carbon atoms in the 1,3-dihydroxy motifs in the C11 core extension (C18) and the O-linked lower side chain (C14'). Since other signals were not discernibly affected and the phenomenon was not observed in the spectrum of 2a, which had been recorded in $\mathrm{CDCl}_{3}$, we suspected that the presence of isomers was not a probable cause for the observation of additional signals in the spectra recorded in acetone- $\mathrm{d}_{6}$; especially, as we had previously encountered a similar behavior with several natural mycolactones, but not with the protected precursors in which hydroxy groups were masked. We thus hypothesized that slow H/D-exchange could be responsible for the observed peak splitting. 

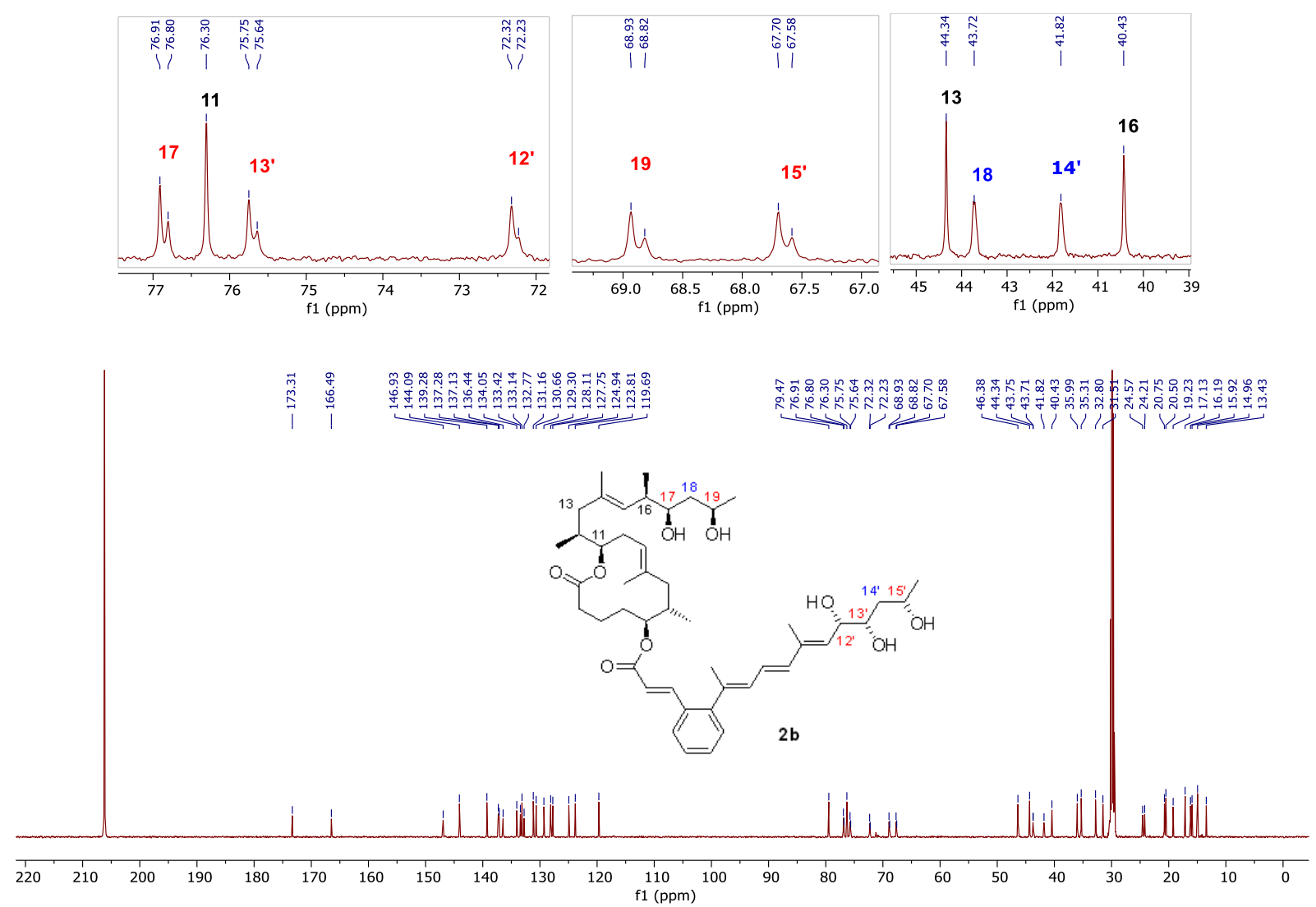

Figure S4: ${ }^{13} \mathrm{C}-\mathrm{NMR}$ spectrum of compound $\mathbf{2 b}$ in acetone- $\mathrm{d}_{6}$. Affected peaks are highlighted in red and blue.

Signals are assigned to the corresponding carbon atoms using mycolactone numbering.
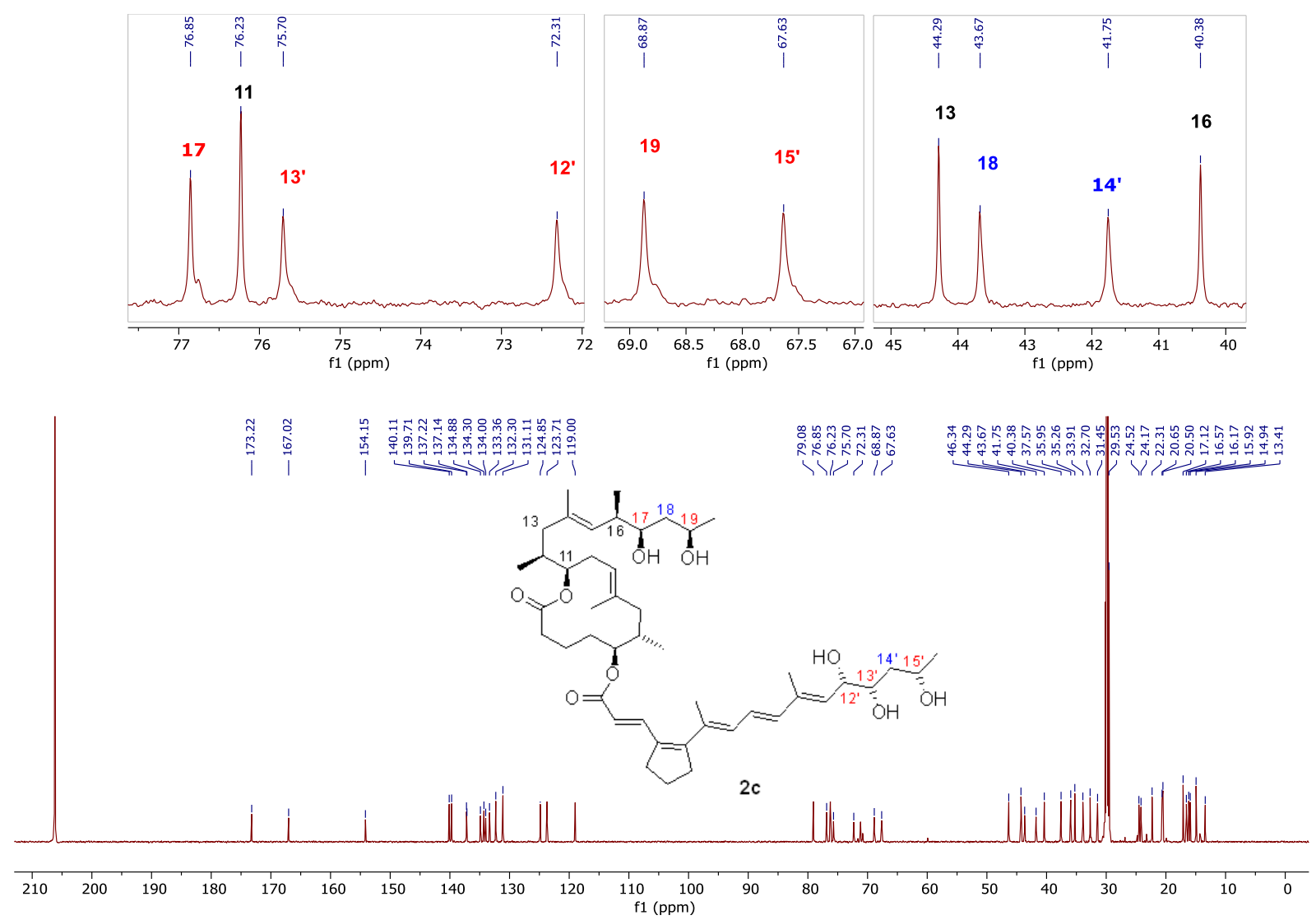

Figure S5: ${ }^{13} \mathrm{C}-\mathrm{NMR}$ spectrum of compound $2 \mathrm{c}$ in acetone- $\mathrm{d}_{6}$. Affected peaks are highlighted in red and blue. Signals are assigned to the corresponding carbon atoms using mycolactone numbering. 
To further investigate the above phenomenon, ${ }^{13} \mathrm{C}$-NMR spectra of model compound $\mathbf{S 1 8}$, an intermediate in the synthesis of C20-hydroxylated mycolactone analogs, ${ }^{11-13}$ were recorded in different deuterated solvents $\left(\mathrm{CDCl}_{3}\right.$, acetone- $\left.\mathrm{d}_{6}, \mathrm{CD}_{3} \mathrm{OD}, \mathrm{CDCl}_{3}+\mathrm{CD}_{3} \mathrm{OD}\right)$. No additional peaks were observed in the ${ }^{13} \mathrm{C}-\mathrm{NMR}$ spectra of diol S18 when recorded in deuterated chloroform (Figure S6), confirming the stereochemical purity of the compound. Similarly, no additional signals were observed in $\mathrm{CD}_{3} \mathrm{OD}$ (not shown).

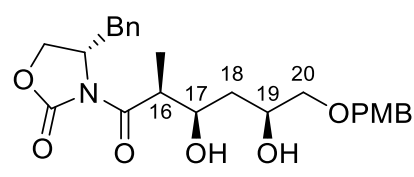

S18
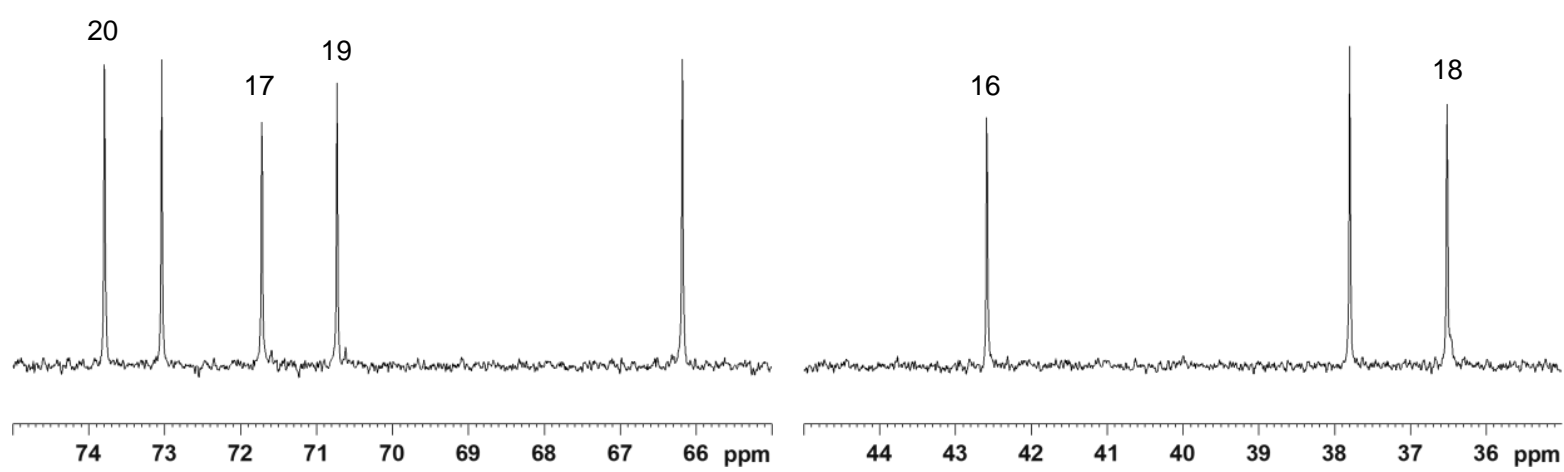

Figure S6. ${ }^{13} \mathrm{C}$-NMR spectra of diol $\mathbf{S 1 8}$ in $\mathrm{CDCl}_{3}$. Only regions where peak splitting had been observed in the spectra of mycolactone analogs in acetone- $\mathrm{d}^{6}$ are shown. Signals are assigned to the corresponding carbon atoms using mycolactone numbering.

Recording the ${ }^{13} \mathrm{C}-\mathrm{NMR}$ spectrum of compound $\mathbf{S 1 8}$ in acetone- $\mathrm{d}_{6}$, however, gave an additional set of upfield-shifted signals for atoms C16 to C20 (mycolactone numbering, Figure S7). 

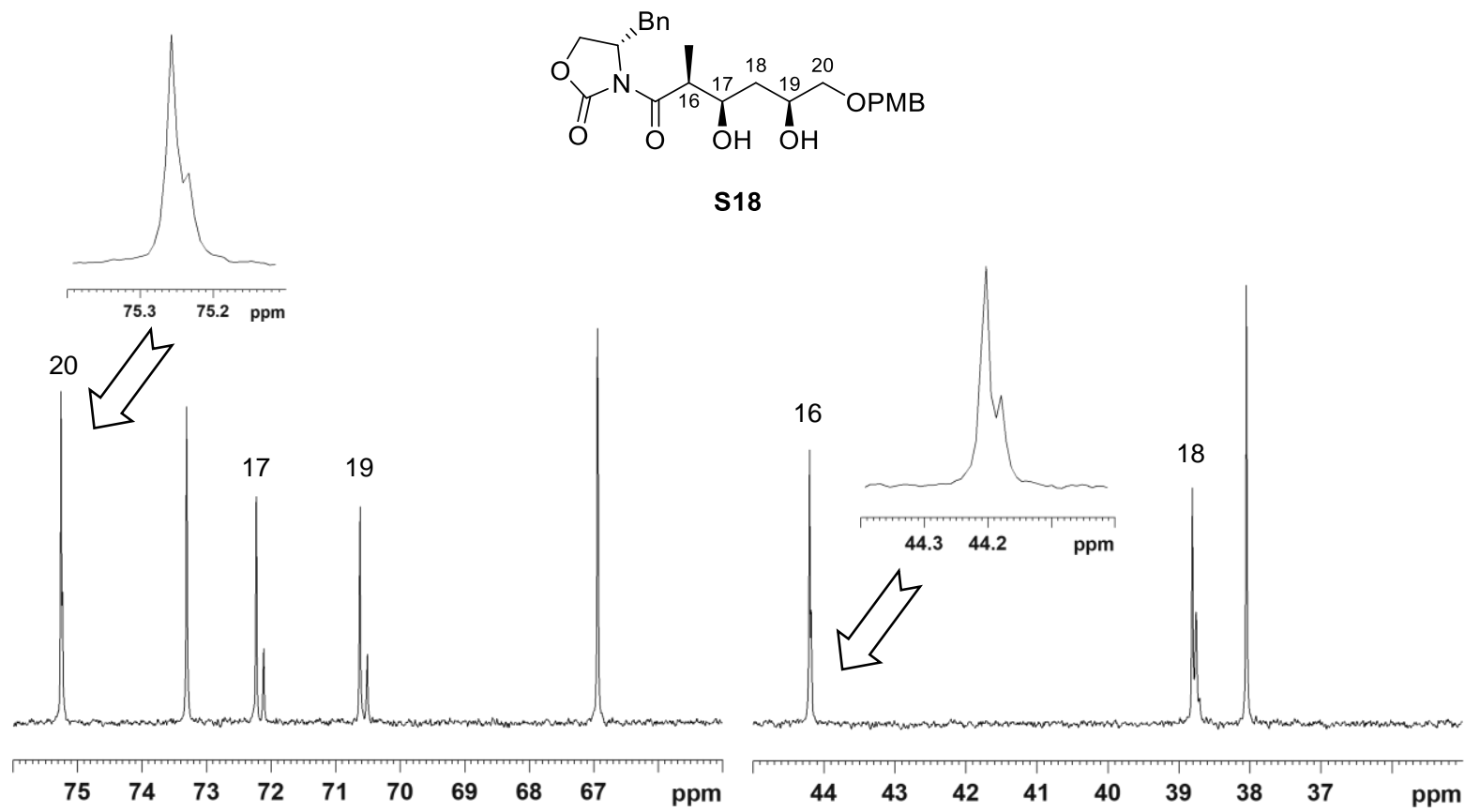

Figure S7. ${ }^{13} \mathrm{C}-\mathrm{NMR}$ spectra of compound $\mathbf{S 1 8}$ in acetone- $\mathrm{d}_{6}$. Only regions where the shifts are typically occurring are shown. Signals are assigned to the corresponding carbon atoms using mycolactone numbering.

The shift difference between the major and the minor peak increased when the respective carbon atom was located closer to one or both hydroxy groups (Table S2), resulting in the following order: C17 C19> C18 > C16 C20.

Table S2. Chemical shifts of C16-C20 in S18 in acetone- $\mathrm{d}_{6} .{ }^{\mathrm{a}}$

\begin{tabular}{cccc}
\hline $\mathbf{C}$ & $\begin{array}{c}\text { Major signal } \\
\boldsymbol{\delta}[\mathbf{p p m}]\end{array}$ & $\begin{array}{c}\text { Minor signal } \\
\delta[\mathbf{p p m}]\end{array}$ & $\begin{array}{c}\boldsymbol{\Delta}^{\mathbf{b}} \\
{[\mathbf{p p m}]}\end{array}$ \\
\hline $\mathbf{1 6}$ & 44.202 & 44.179 & 0.023 \\
$\mathbf{1 7}$ & 72.230 & 72.114 & 0.116 \\
$\mathbf{1 8}$ & 38.809 & 38.758 & 0.052 \\
$\mathbf{1 9}$ & 70.620 & 70.509 & 0.111 \\
$\mathbf{2 0}$ & 75.255 & 75.234 & 0.021 \\
\hline
\end{tabular}

${ }^{\mathrm{a}}$ Chemical shifts $(\delta)$ for $\mathbf{S 1 8}$ were recorded in acetone- $\mathrm{d}_{6}$ on a Bruker AV-400 $400 \mathrm{MHz}$ spectrometer at room temperature. ${ }^{b} \Delta$ values were calculated as follows: $\Delta=\delta_{\text {major signal }}-\delta_{\text {minor signal. }}$.

If the spectra of compound $\mathbf{S 1 8}$ were recorded in $\mathrm{CDCl}_{3}$ and a drop of $\mathrm{CD}_{3} \mathrm{OD}$ was added, additional signals was observed for $\mathrm{C} 17$ and $\mathrm{C} 19$; the signals for $\mathrm{C} 16$ and $\mathrm{C} 20$ were only slightly broadened, while at least 3 different signals were observed for C18 (not shown).

No abnormalities were observed in the ${ }^{1} \mathrm{H}-\mathrm{NMR}$ spectra of $\mathbf{S 1 8}$ in any of the above solvents. 
The above data support the hypothesis that the occurrence of additional signals in the ${ }^{13} \mathrm{C}-\mathrm{NMR}$ spectra of mycolactones arises from hydrogen-deuterium exchange and the resulting isotope effects on the ${ }^{13} \mathrm{C}-\mathrm{NMR}$ signals. Notably, the difference in ${ }^{13} \mathrm{C}-\mathrm{NMR}$ shifts between protonated hydroxy groups (R-OH) and their deuterated analogs (R-OD) has been exploited by Pfeffer and co-workers for the structural assignment of carbohydrates. ${ }^{14-16}$ Similar effects have also been described for carbon atoms in a variety of other hydroxy-substituted compounds, e.g. in the $\alpha-$ and $\beta$-position of aliphatic alcohols, ${ }^{17}$ in the ipso- and ortho-position in alkyl substituted phenols, ${ }^{17}$ in enolic $\beta$-diketones, ${ }^{18}$ in Schiff bases derived from 1-hydroxy-2-acetonaphthone and 2-hydroxy-1-naphthaldehyde, ${ }^{19}$ and in 1,2-O-isopropylidene- $\alpha$-D-glucofuranose (MAGF). ${ }^{20}$ Notably, in the latter case, the deuterium isotope shift was initially observed after dissolving MAGF in DMSO- $\mathrm{d}_{6} /$ acetone- $_{6}(1: 1)$ for NMR analysis. ${ }^{20}$

In accordance with our observations for compounds $\mathbf{2 b / c}$ and $\mathbf{S 1 8}$, differential isotope shifts are generally most pronounced for the carbon atoms directly attached to the hydroxy group, but are also observed for carbon atoms in the $\beta$-position. ${ }^{14,20}$ These previous findings are in line with the solvent effects observed in our own investigations: While proton-deuterium exchange does not occur in $\mathrm{CDCl}_{3}$, it is very fast in $\mathrm{CD}_{3} \mathrm{OD}$. Consequently, only a single set of signals is observed in either of these solvents. In contrast, acetone- $\mathrm{d}_{6}$ promotes a slow exchange via the protic enol form. If an alcohol is dissolved in a large excess of acetone- $\mathrm{d}_{6}$ for extended time periods (i.e. several days), an almost complete proton-deuterium exchange should result. We speculate that this is why we did not always observe this effect in our previous synthetic work on $\mathbf{1 a} / \mathbf{b} .^{2,11,12}$ If a solution of $\mathbf{S 1 8}$ in $\mathrm{CDCl}_{3}$ is spiked with only trace amounts of $\mathrm{CD}_{3} \mathrm{OD}$, an equilibrium state of protonated and deuterated hydroxy groups should result, which is also in accordance with our experiments. Thus, deuterium-induced differential ${ }^{13} \mathrm{C}-\mathrm{NMR}$ shifts provide a consistent explanation for the observed phenomenon, which is thus not caused by isomeric impurities in our preparations of $\mathbf{2 b}$ and $\mathbf{2 c}$. 


\subsection{Note added during revision}

As part of the review process for this manuscript, one of the reviewers recommended to determine the effect of the addition of $\mathrm{D}_{2} \mathrm{O}$ on the ${ }^{13} \mathrm{C}-\mathrm{NMR}$ spectra of compounds $\mathbf{2 b} / \mathbf{2} \mathbf{c}$ in acetone- $\mathrm{d}_{6}$. This reviewer raised the possibility that the satellite signals observed for selected carbons in the ${ }^{13} \mathrm{C}$-NMR spectra of these compounds were in fact due to the presence of minor isomers. While we believe that the data presented for the model compound S18 leave little doubt that these satellite signals arise from the use of acetone- $\mathrm{d}_{6}$ as the NMR solvent, we tried to comply we the reviewer's recommendation. However, in order to do so without having to go through the re-synthesis of the compound, we have used an impure sample of $\mathbf{2} \mathbf{b}$ that was contaminated with TBAF residues from the final deprotection and also some minor isomers. Given the small amount of compound still available and based on past experience with losses of material during the preparative HPLC purification of other mycolactone variants, we felt that the information gained from spectra of this material would serve the purpose at hand. The ${ }^{13} \mathrm{C}$ spectrum of this impure preparation of $\mathbf{2} \mathbf{b}$ was then recorded in acetone- $\mathrm{d}_{6}$ and re-recorded after addition of $\mathrm{D}_{2} \mathrm{O}$. The interpretation of the data was complicated by (1) minor shifts of some signals in the spectrum in acetone- $\mathrm{d}_{6}$ compared to the spectrum originally obtained with $\mathbf{2 b}$; and (2) the fact that satellite signals were less pronounced than previously observed for some carbons. While we have no clear explanation for the first observation (although it may simple be a consequence of the use of a different batch of NMR solvent), the second observation is not surprising. We have found the intensities of these satellite signals to vary also in our previous mycolactone work. Notwithstanding these difficulties, satellite signals were clearly reduced or eliminated in the presence of $\mathrm{D}_{2} \mathrm{O}$, which once again corroborates our conclusion that the satellite signals observed (only) for carbons attached to hydroxy groups or those connected to such carbons cannot be ascribed to the presence of other isomers.

Finally, we also emphasize that not satellite signals or line broadening was observed in spectra of the fully protected precursors S16 and S17, respectively. Thus, if our observations were indicative of the presence of other isomers in $\mathbf{2 b}$ and $\mathbf{2} \mathbf{c}$, it would imply that significant epimerization should have occurred at all hydroxy-bearing carbons in the final deprotection steps, which would be difficult to explain. 


\section{References}

(1) Mayer, M. U.; Breslow, R. Antihydrophobic Evidence for the Single Electron Transfer Mechanism of Nucleophilic Substitution. J. Am. Chem. Soc. 1998, 120 (35), 9098-9099. https://doi.org/10.1021/ja982137j.

(2) Gersbach, P.; Jantsch, A.; Feyen, F.; Scherr, N.; Dangy, J.-P.; Pluschke, G.; Altmann, K.-H. A Ring-Closing Metathesis (RCM)-Based Approach to Mycolactones A/B. Chem. - Eur. J. 2011, 17 (46), 13017-13031. https://doi.org/10.1002/chem.201101799.

(3) Fujino, D.; Yorimitsu, H.; Osuka, A. Regiocontrolled Palladium-Catalyzed Arylative Cyclizations of Alkynols. J. Am. Chem. Soc. 2014, 136 (17), 6255-6258. https://doi.org/10.1021/ja5029028.

(4) Spivey, A. C.; McKendrick, J.; Srikaran, R.; Helm, B. A. Solid-Phase Synthesis of an A-B Loop Mimetic of the C\&3 Domain of Human IgE: Macrocyclization by Sonogashira Coupling. J. Org. Chem. 2003, 68 (5), 1843-1851. https://doi.org/10.1021/jo026693e.

(5) Wang, X.; Silverman, R. B. 2-(Iodoethenyl)Benzylamines as Potential Probes for Radical Intermediates Formed during Monoamine Oxidase Catalyzed Oxidations. $J$. Org. Chem. 1998, 63 (21), 7357-7363. https://doi.org/10.1021/jo980976i.

(6) Wang, G.; Zhu, G.; Negishi, E. Zirconium-Catalyzed Methylalumination of Heterosubstituted Arylethynes: Factors Affecting the Regio-, Stereo-, and Chemoselectivities. J. Organomet. Chem. 2007, 692 (21), 4731-4736. https://doi.org/10.1016/j.jorganchem.2007.05.052.

(7) Song, F.; Fidanze, S.; Benowitz, A. B.; Kishi, Y. Total Synthesis of the Mycolactones. Org. Lett. 2002, 4 (4), 647-650. https://doi.org/10.1021/ol0172828.

(8) Chany, A.-C.; Casarotto, V.; Schmitt, M.; Tarnus, C.; Guenin-Macé, L.; Demangel, C.; Mirguet, O.; Eustache, J.; Blanchard, N. A Diverted Total Synthesis of Mycolactone Analogues: An Insight into Buruli Ulcer Toxins. Chem. - Eur. J. 2011, 17 (51), 1441314419. https://doi.org/10.1002/chem.201102542.

(9) Su, N.; Theorell, J. A.; Wink, D. J.; Driver, T. G. Copper-Catalyzed Formation of $\alpha-$ Alkoxycycloalkenones from N-Tosylhydrazones. Angew. Chem. Int. Ed. 2015, 54 (44), 12942-12946. https://doi.org/10.1002/anie.201505993.

(10) Ohe, K.; Miki, K.; Yokoi, T.; Nishino, F.; Uemura, S. Novel Pyranylidene Complexes from Group 6 Transition Metals and $\beta$-Ethynyl $\alpha, \beta$-Unsaturated Carbonyl Compounds. Organometallics 2000, 19 (25), 5525-5528. https://doi.org/10.1021/om0006763.

(11) Scherr, N.; Gersbach, P.; Dangy, J.-P.; Bomio, C.; Li, J.; Altmann, K.-H.; Pluschke, G. Structure-Activity Relationship Studies on the Macrolide Exotoxin Mycolactone of Mycobacterium Ulcerans. PLoS Negl. Trop. Dis. 2013, 7 (3), e2143. https://doi.org/10.1371/journal.pntd.0002143.

(12) Gehringer, M.; Altmann, K.-H. The Chemistry and Biology of Mycolactones. Beilstein J. Org. Chem. 2017, 13 (1), 1596-1660. https://doi.org/10.3762/bjoc.13.159.

(13) Bieri, R.; Scherr, N.; Ruf, M.-T.; Dangy, J.-P.; Gersbach, P.; Gehringer, M.; Altmann, K.-H.; Pluschke, G. The Macrolide Toxin Mycolactone Promotes Bim-Dependent Apoptosis in Buruli Ulcer through Inhibition of mTOR. ACS Chem. Biol. 2017, 12 (5), 1297-1307. https://doi.org/10.1021/acschembio.7b00053.

(14) Pfeffer, P. E.; Valentine, K. M.; Parrish, F. W. Deuterium-Induced Differential Isotope Shift Carbon-13 NMR. 1. Resonance Reassignments of Mono- and Disaccharides. J. Am. Chem. Soc. 1979, 101 (5), 1265-1274. https://doi.org/10.1021/ja00499a036.

(15) Pfeffer, P. E.; Parrish, F. W.; Unruh, J. Effects of Carbohydrate-Structure Changes on Induced Shifts in Differential Isotope-Shift 13C-N.M.R. Carbohydr. Res. 1980, 84 (1), 13-23. https://doi.org/10.1016/S0008-6215(00)85425-7. 
(16) Pfeffer, P. E.; Hicks, K. B. Characterization of Keto Disaccharides in Solution by Deuterium-Induced, Differential Isotope-Shift 13C-N.M.R. Spectroscopy. Carbohydr. Res. 1982, 102 (1), 11-22. https://doi.org/10.1016/S0008-6215(00)88046-5.

(17) Newmark, R. A.; Hill, J. R. Use of 13C Isotope Shifts to Assign Carbon Atoms $\alpha$ and $\beta$ (and Para) to a Hydroxy Group in Alkyl Substituted Phenols and Alcohols. Org. Magn. Reson. 1980, 13 (1), 40-44. https://doi.org/10.1002/mrc.1270130109.

(18) Bolvig, S.; Hansen, P. E. Deuterium-Induced Isotope Effects on 13C Chemical Shifts as a Probe for Tautomerism in Enolic $\beta$-Diketones. Magn. Reson. Chem. 1996, 34 (6), 467478. https://doi.org/10.1002/(SICI)1097-458X(199606)34:6<467::AIDOMR906>3.0.CO;2-L.

(19) Dziembowska, T.; Rozwadowski, Z.; Filarowski, A.; Hansen, P. E. NMR Study of Proton Transfer Equilibrium in Schiff Bases Derived from 2-Hydroxy-1Naphthaldehyde and 1-Hydroxy-2-Acetonaphthone. Deuterium Isotope Effects on 13C and 15N Chemical Shifts. Magn. Reson. Chem. 2001, 39 (S1), S67-S80. https://doi.org/10.1002/mrc.949.

(20) Gilbert, A.; Silvestre, V.; Robins, R. J.; Remaud, G. S. Impact of the Deuterium Isotope Effect on the Accuracy of 13C NMR Measurements of Site-Specific Isotope Ratios at Natural Abundance in Glucose. Anal. Bioanal. Chem. 2010, 398 (5), 1979-1984. https://doi.org/10.1007/s00216-010-4167-9. 
5. NMR spectra of new compounds

${ }^{1} H$-NMR of compound 6

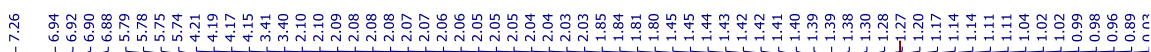<smiles>CCOC(=O)/C=C/C1CCC(CO[Si](C)(C)C(C)(C)C)CC1</smiles>

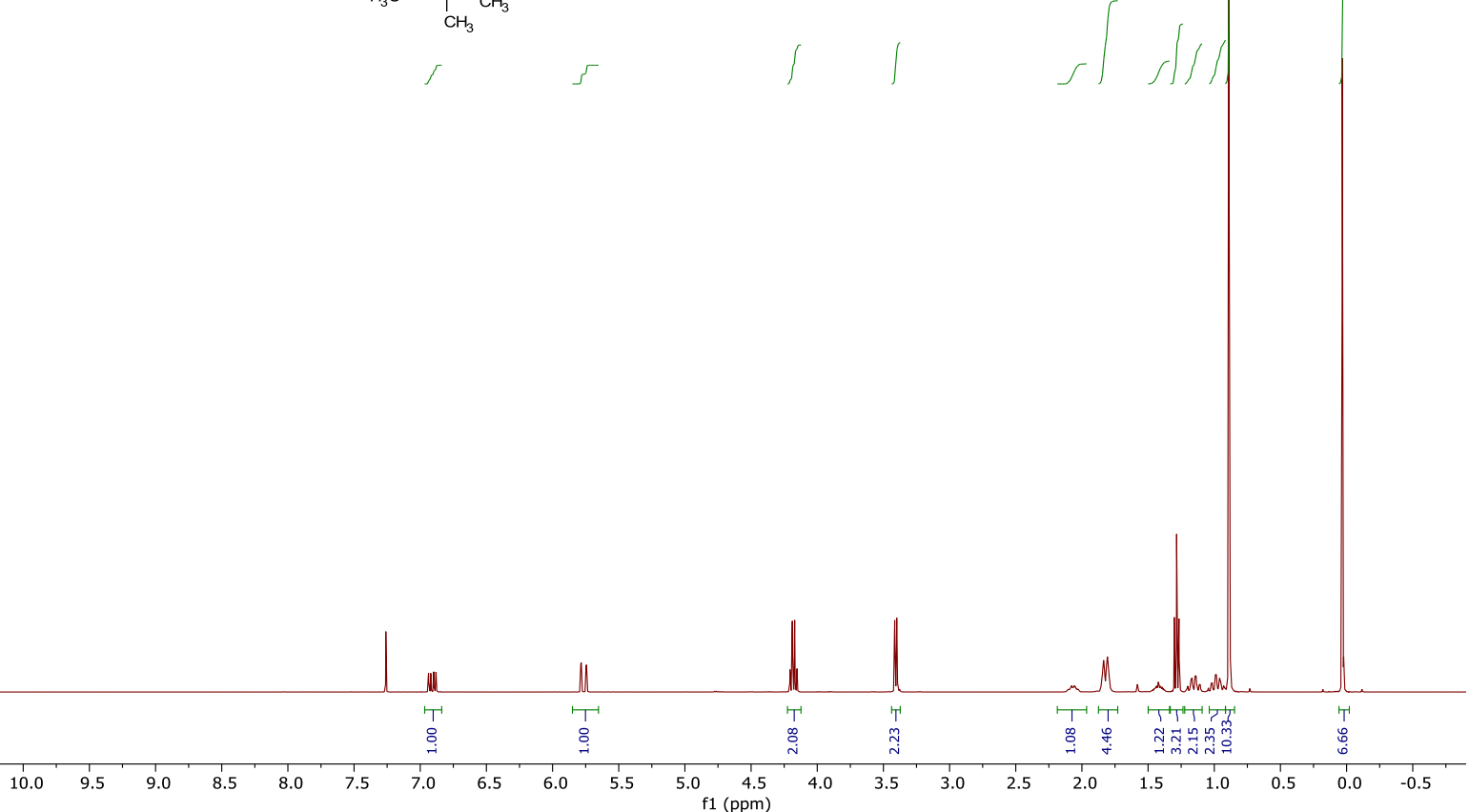

${ }^{13}$ C-NMR of compound 6
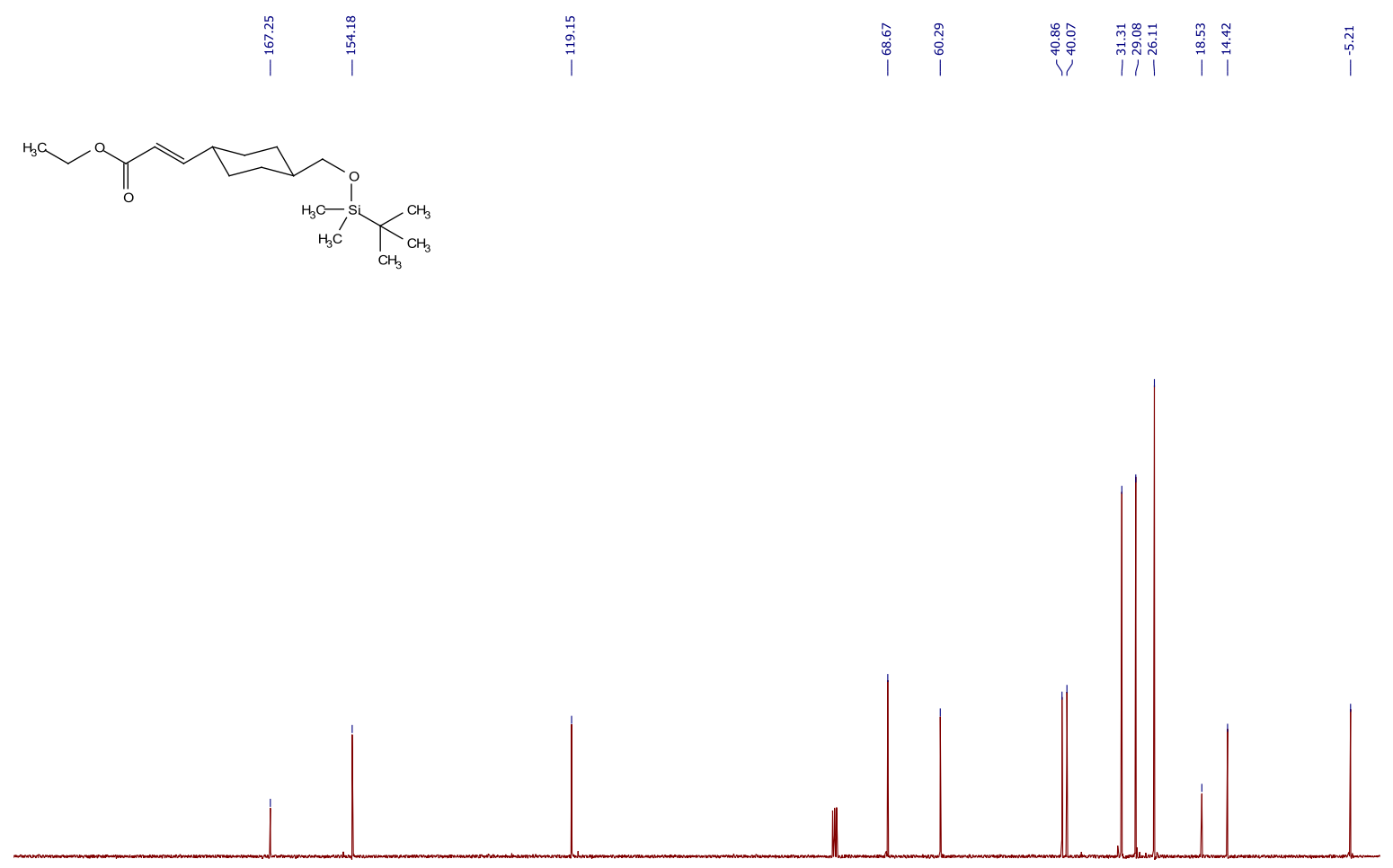

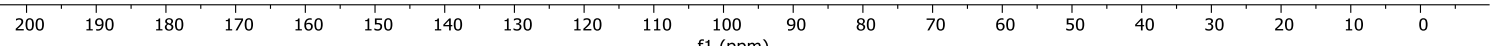




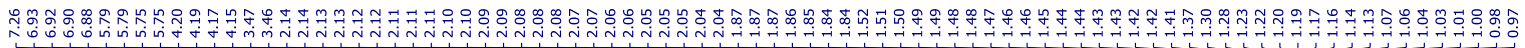<smiles>CCOC(=O)/C=C/C1CCC(CO)CC1</smiles>

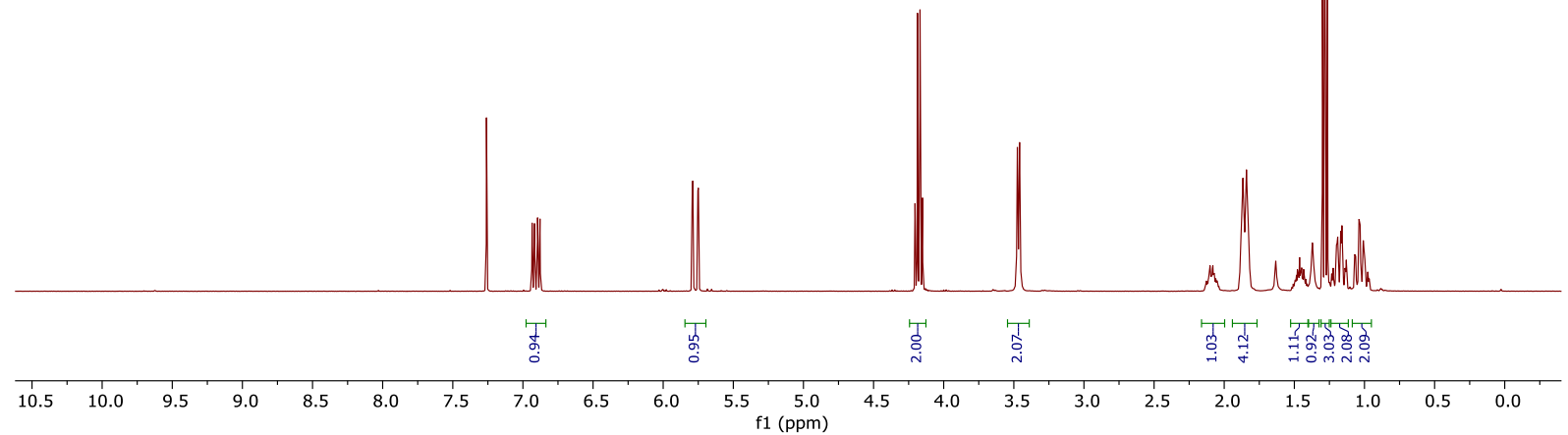

${ }^{13}$ C-NMR of compound $\mathbf{S 3}$

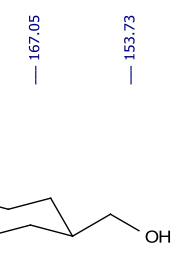<smiles>CCOC(=O)/C=C/C1CCC(CO)CC1</smiles>

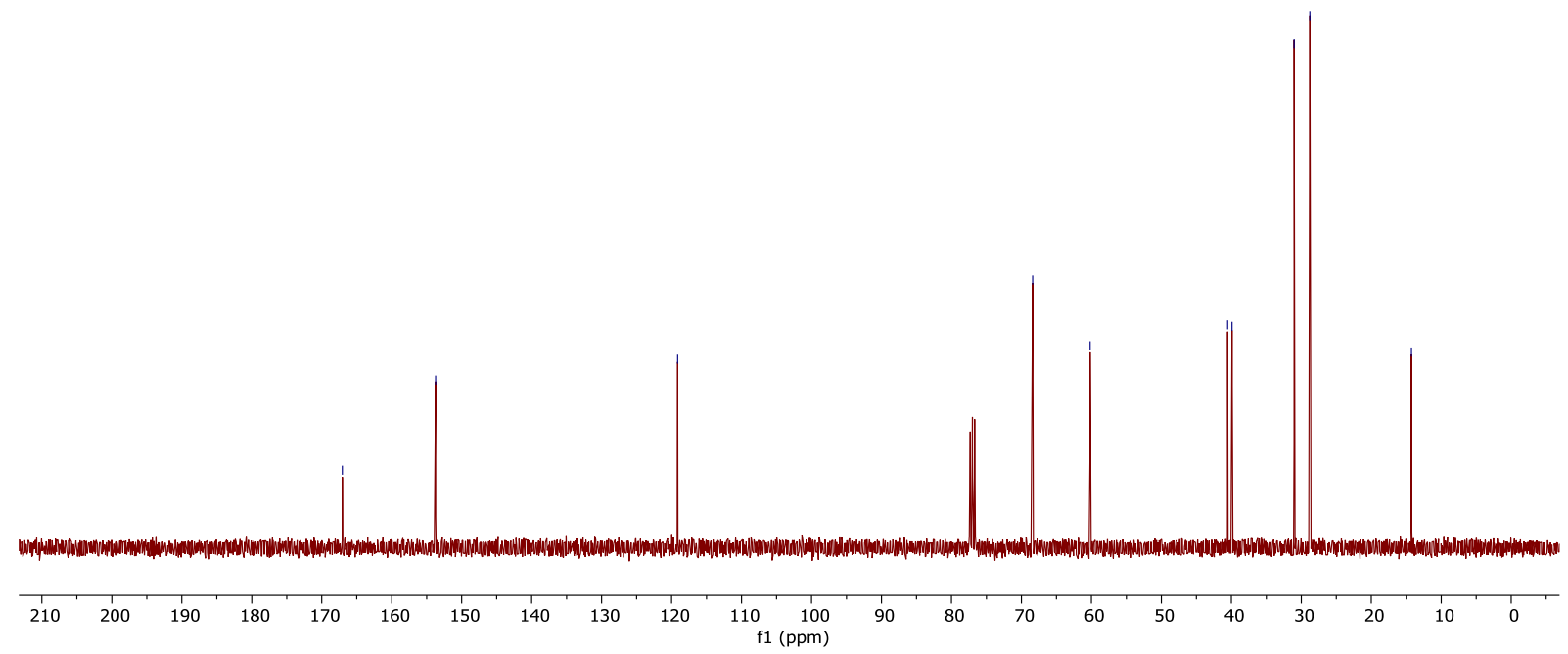




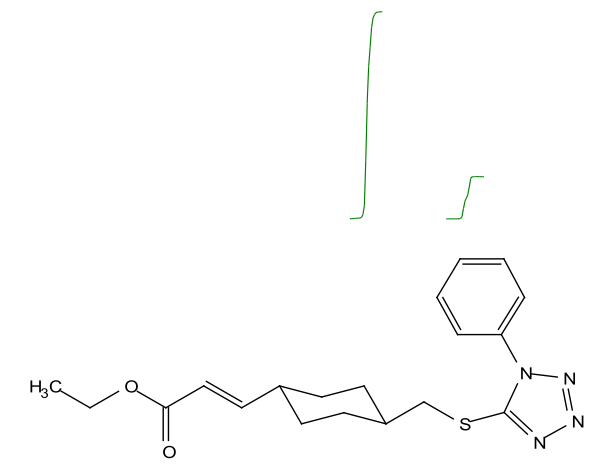

牙选

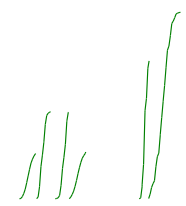

$N \mid$
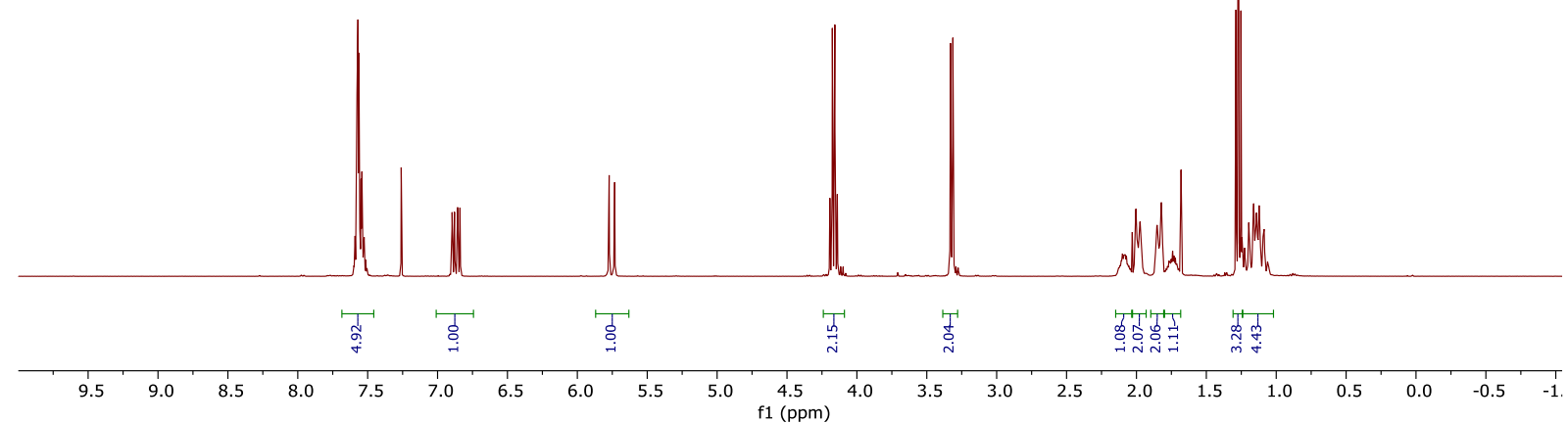

${ }^{13}$ C-NMR of compound $\mathbf{S 4}$
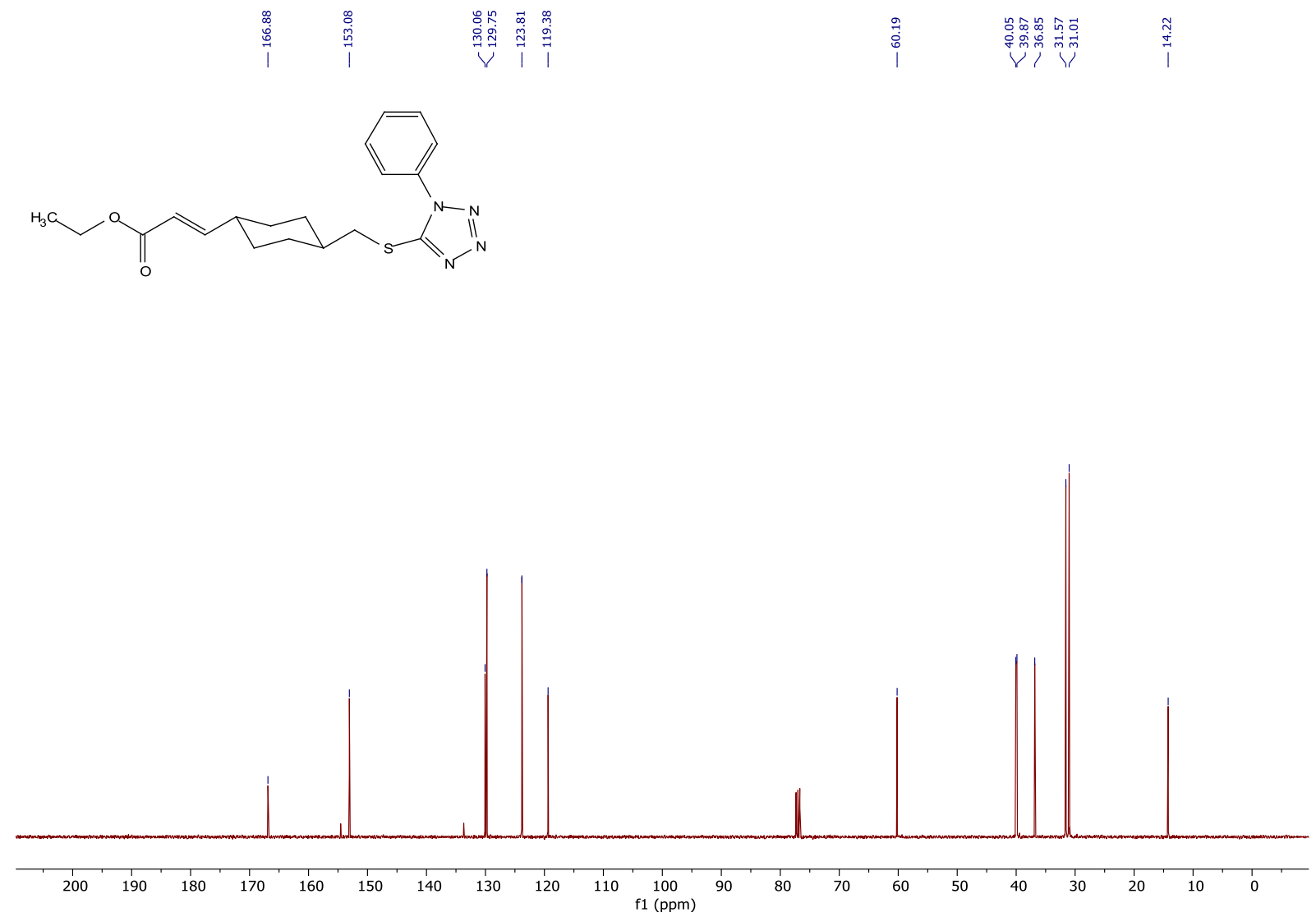

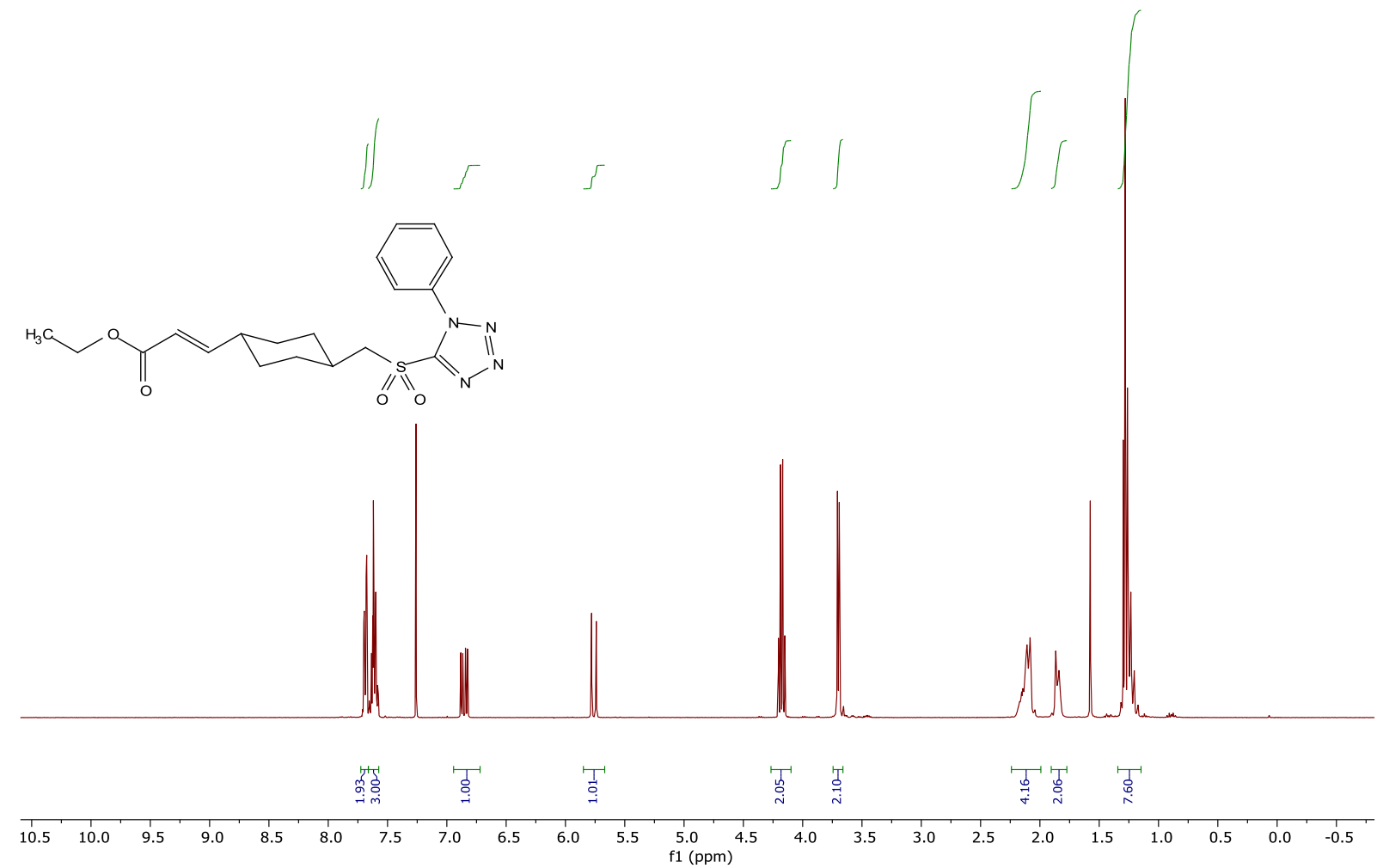

${ }^{13}$ C-NMR of compound 7

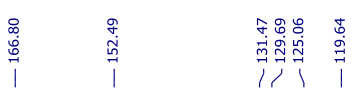
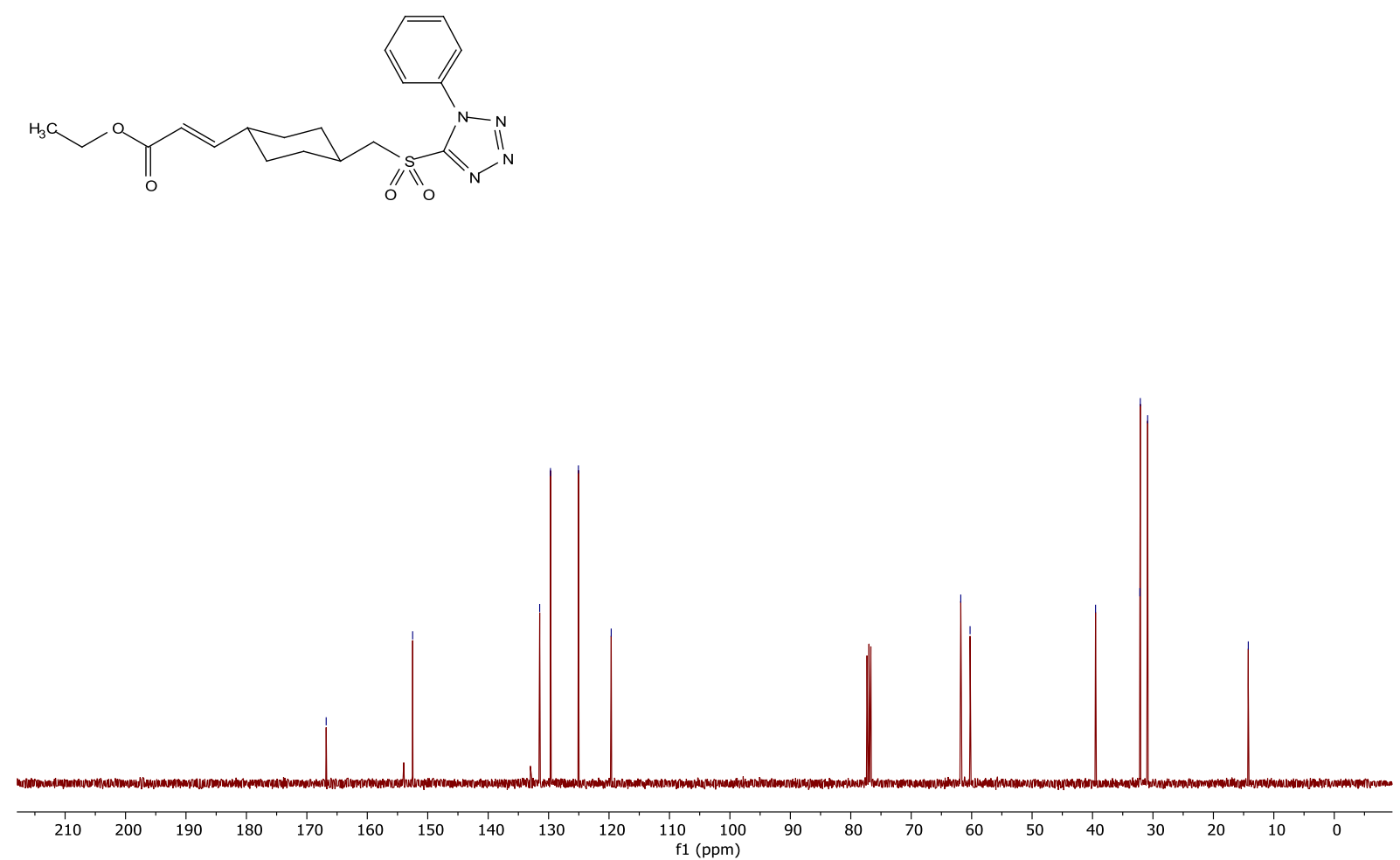


\section{${ }^{1} \mathrm{H}$-NMR of compound $\mathbf{S 5}$}

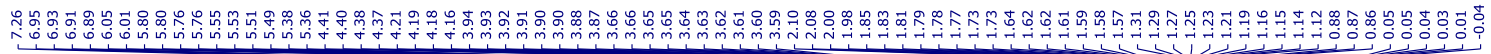

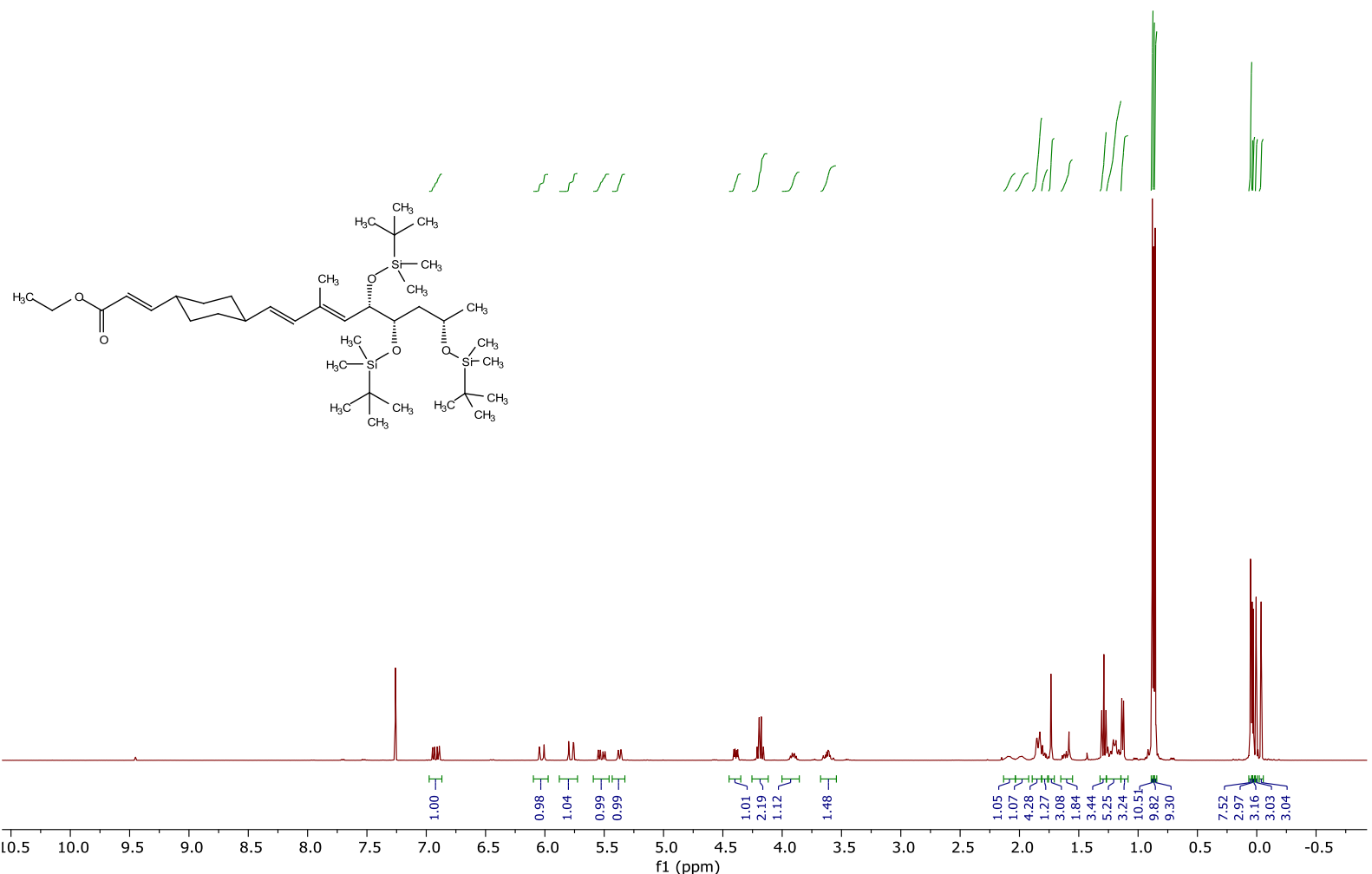

\section{${ }^{13}$ C-NMR of compound S5}
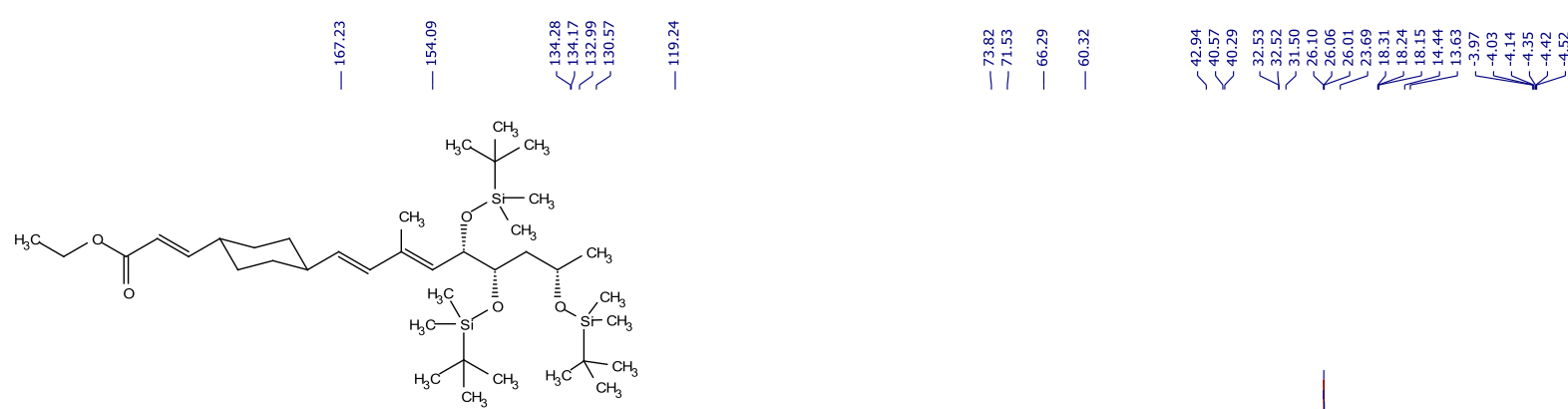


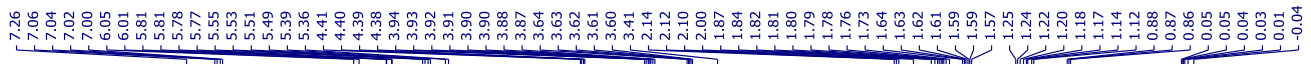

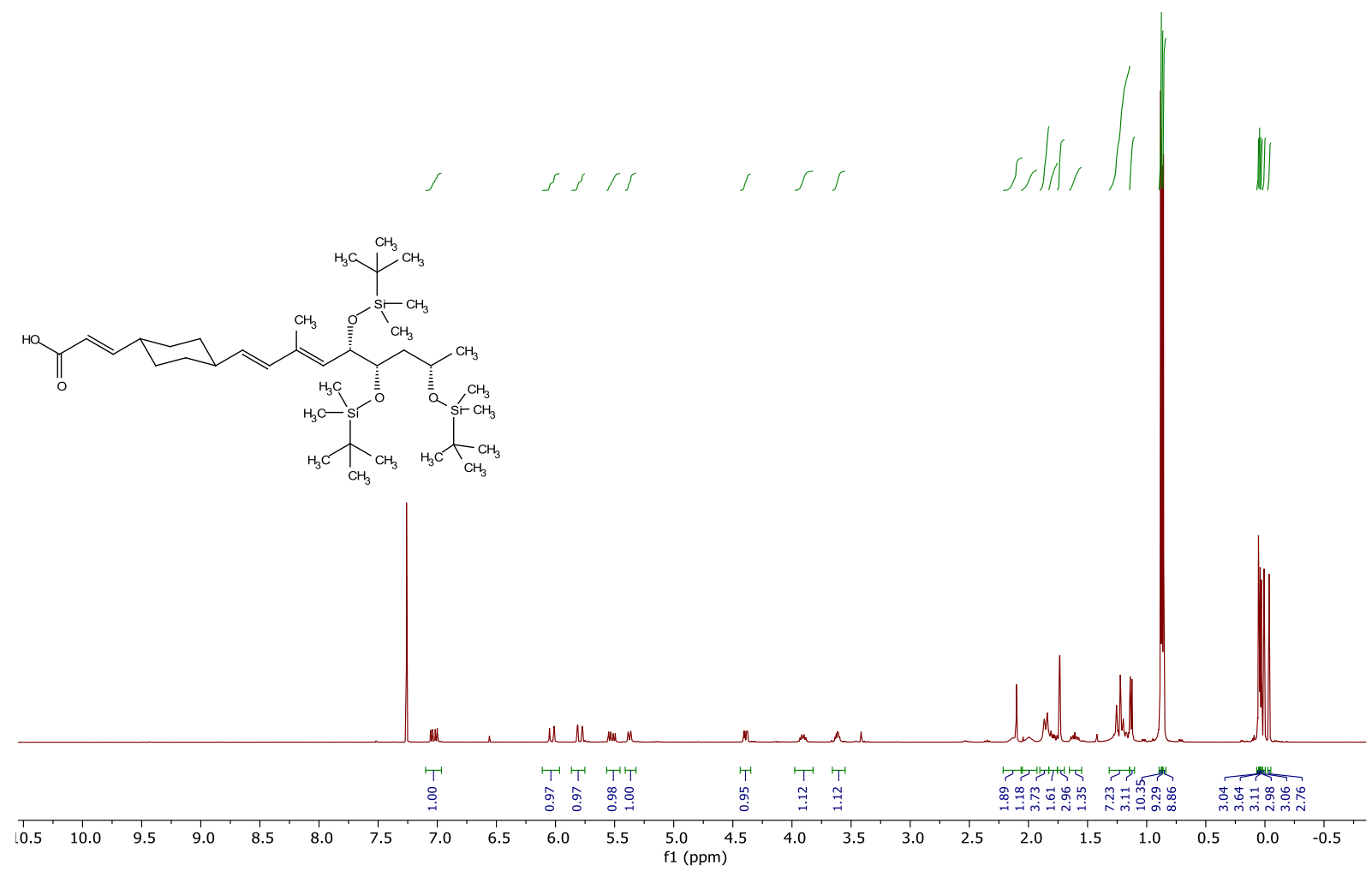

${ }^{13}$ C-NMR of compound $\mathbf{4 a}$

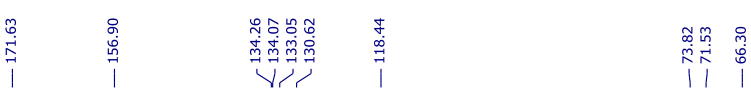

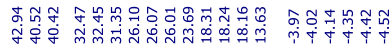
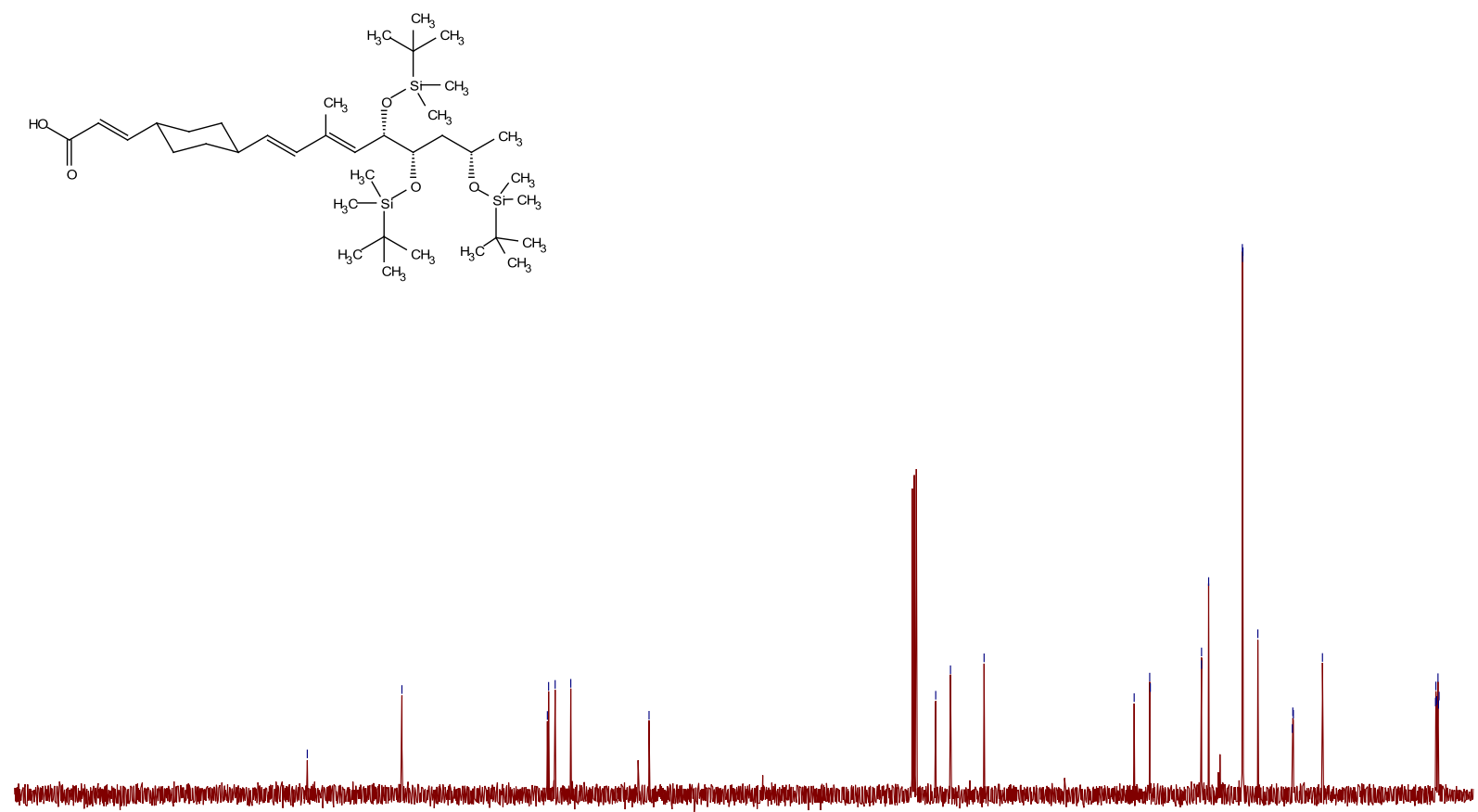

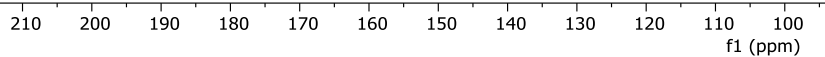


${ }^{1} \mathrm{H}$-NMR of compound $\mathbf{S 8}$

Y.

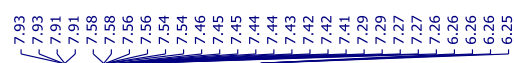

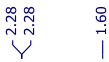

$\overbrace{0}^{\mathrm{CH}_{3}}$
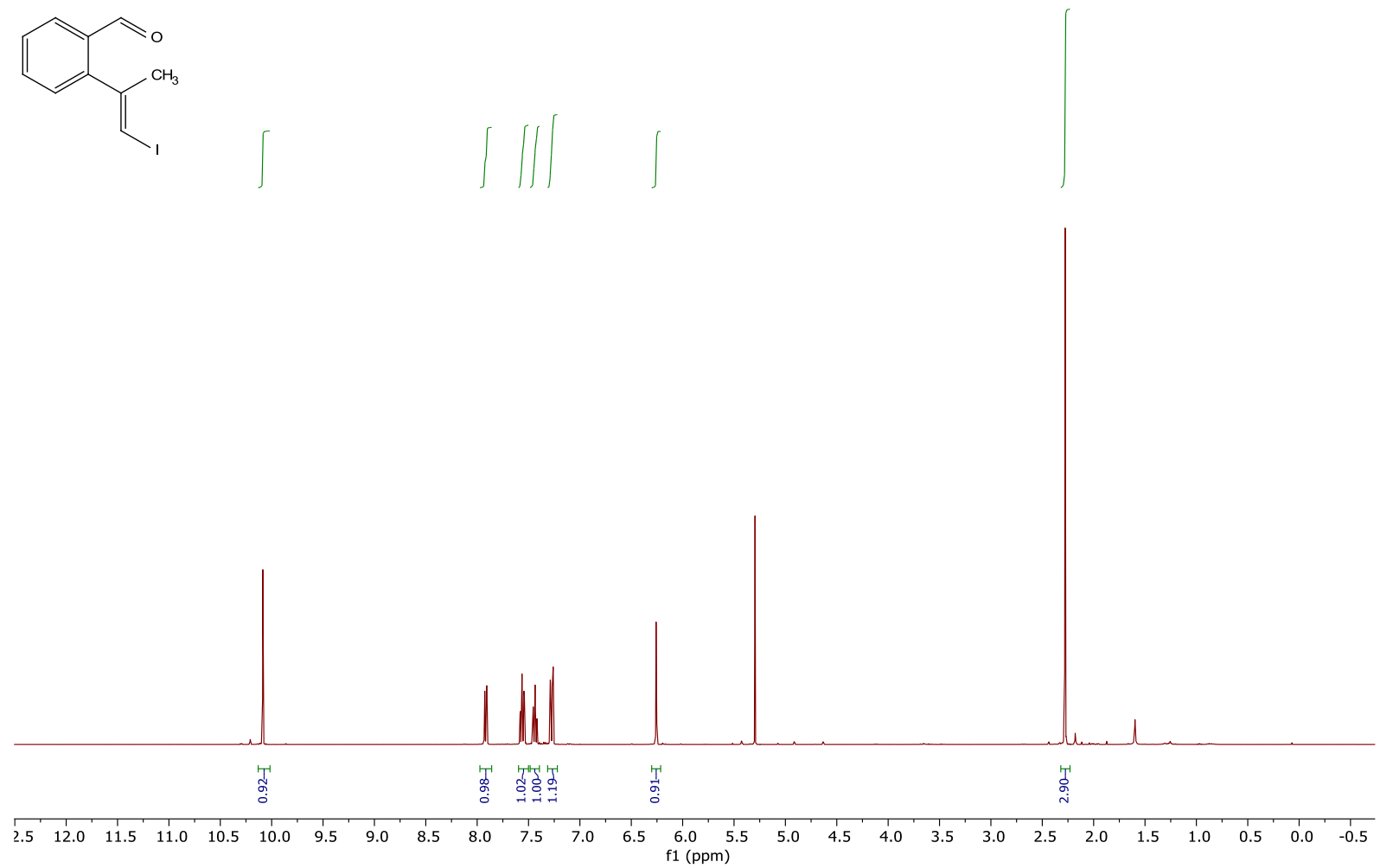

${ }^{13} \mathrm{C}$-NMR of compound $\mathbf{S} 8$

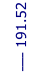

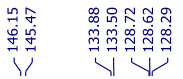

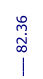

$\underset{\substack{7 \\ i}}{i}$<smiles>O=Cc1ccccc1/C=C/I</smiles>

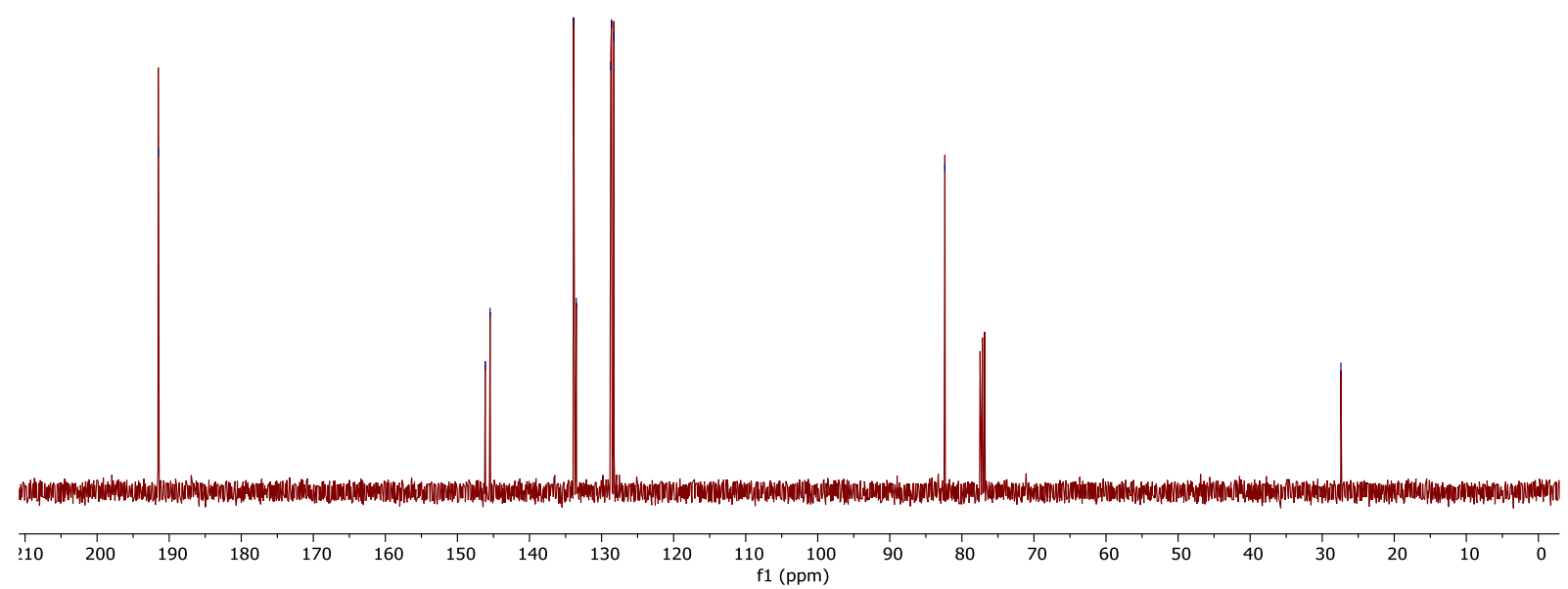


${ }^{1} \mathrm{H}$-NMR of compound 12

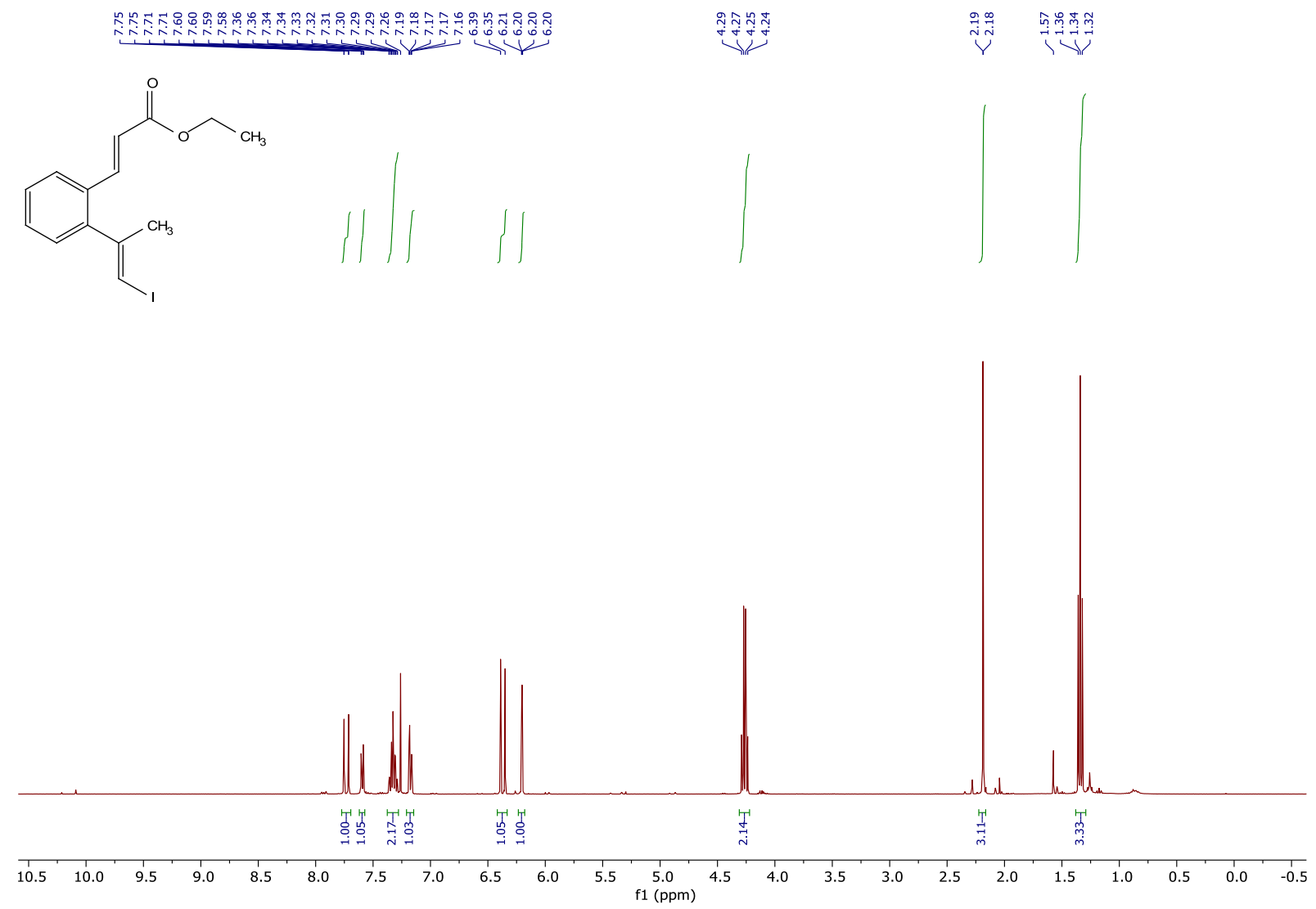

${ }^{13}$ C-NMR of compound 12
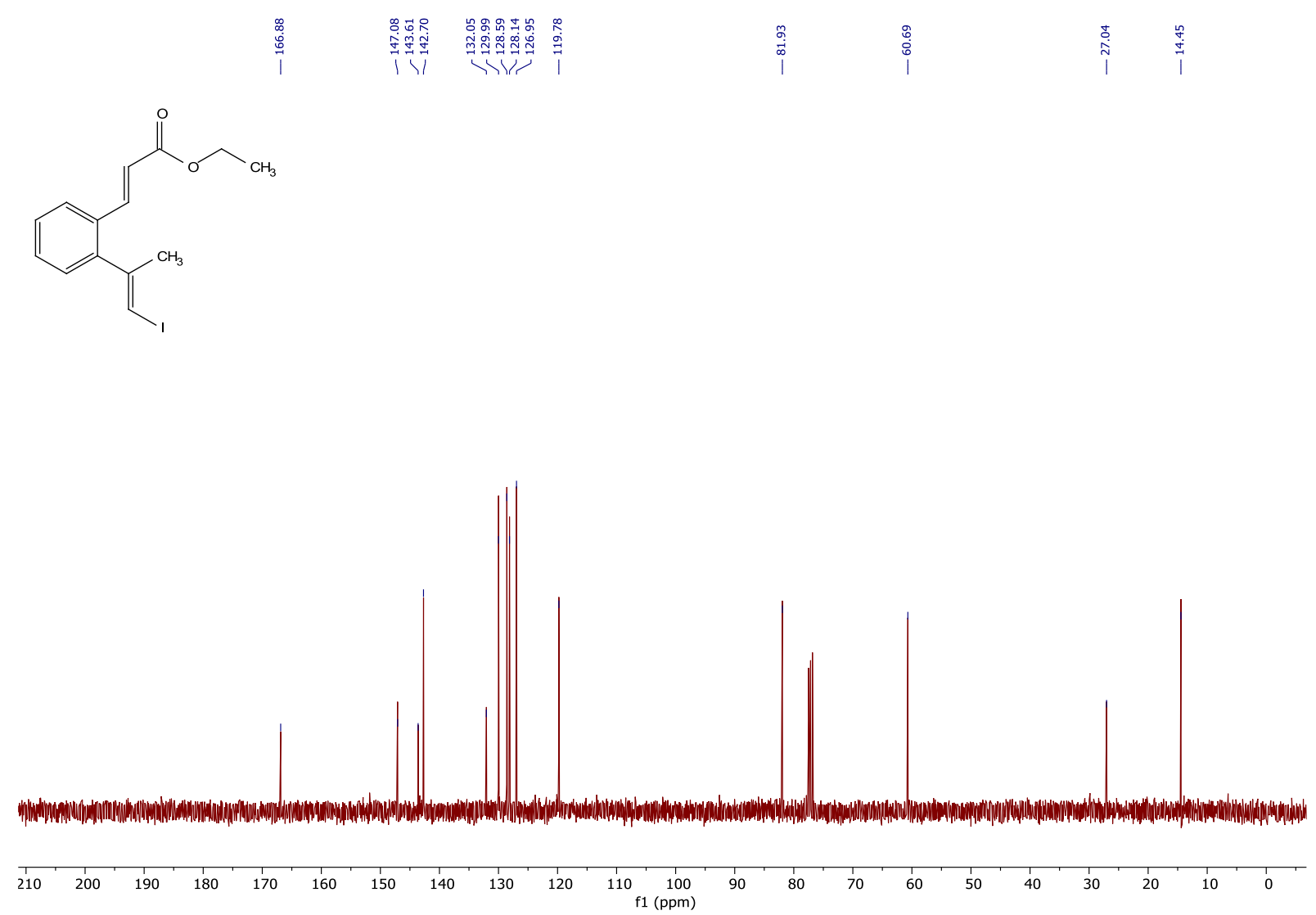
${ }^{1} \mathrm{H}$-NMR of compound 13

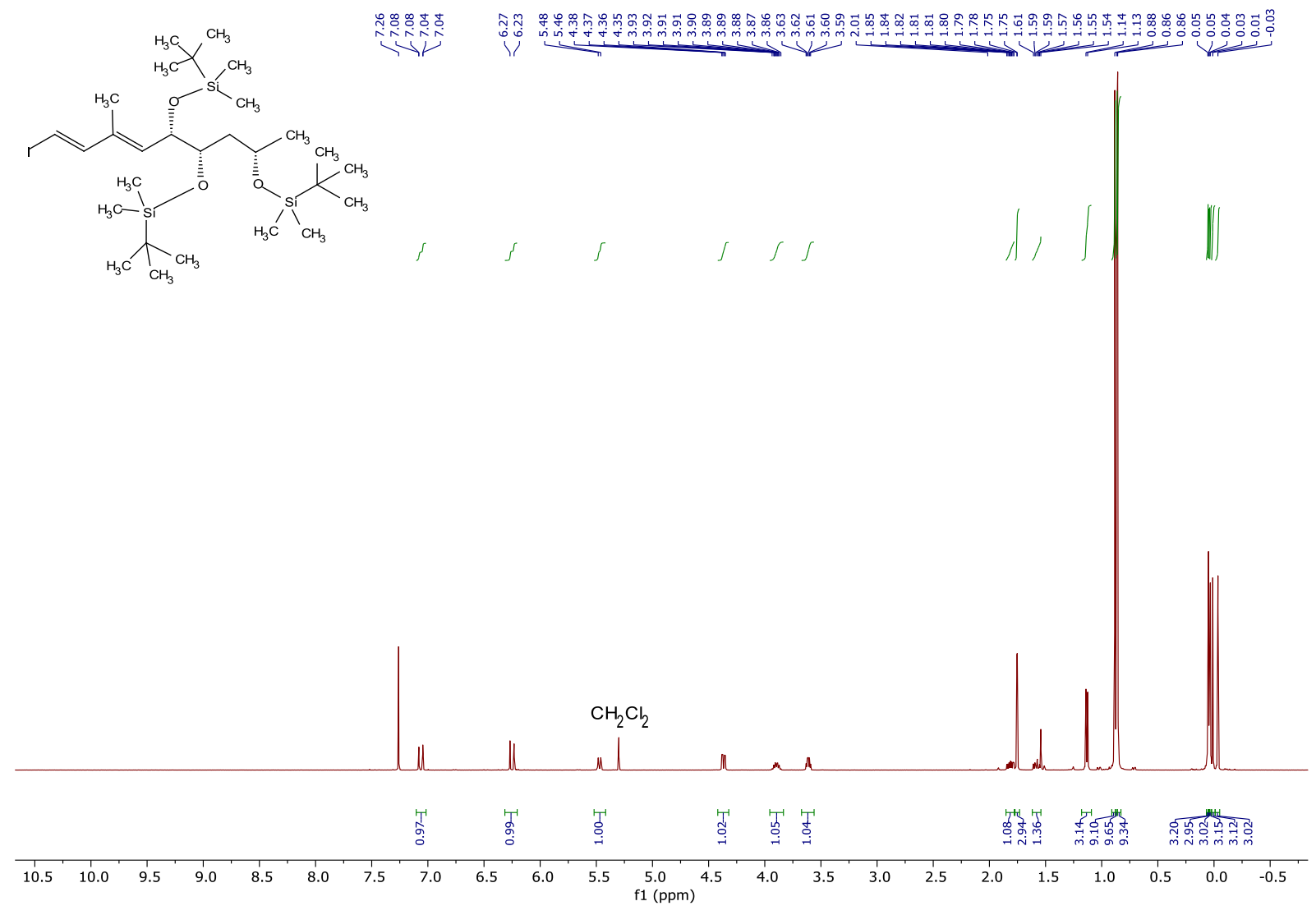

${ }^{13}$ C-NMR of compound 13

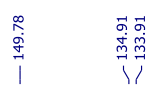

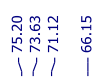

实

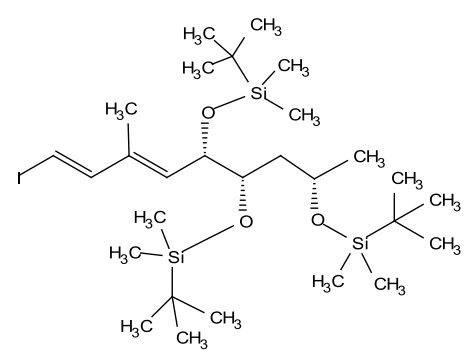

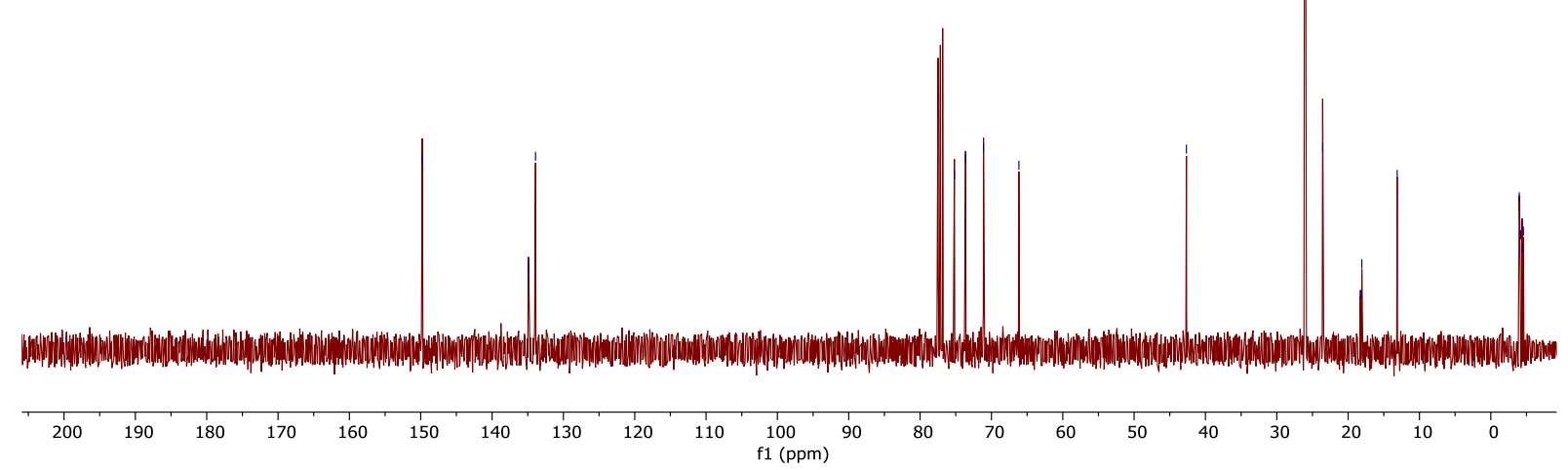


${ }^{1} H$-NMR of compound 14 (crude, contains Bu3 $\left.S n X\right)$

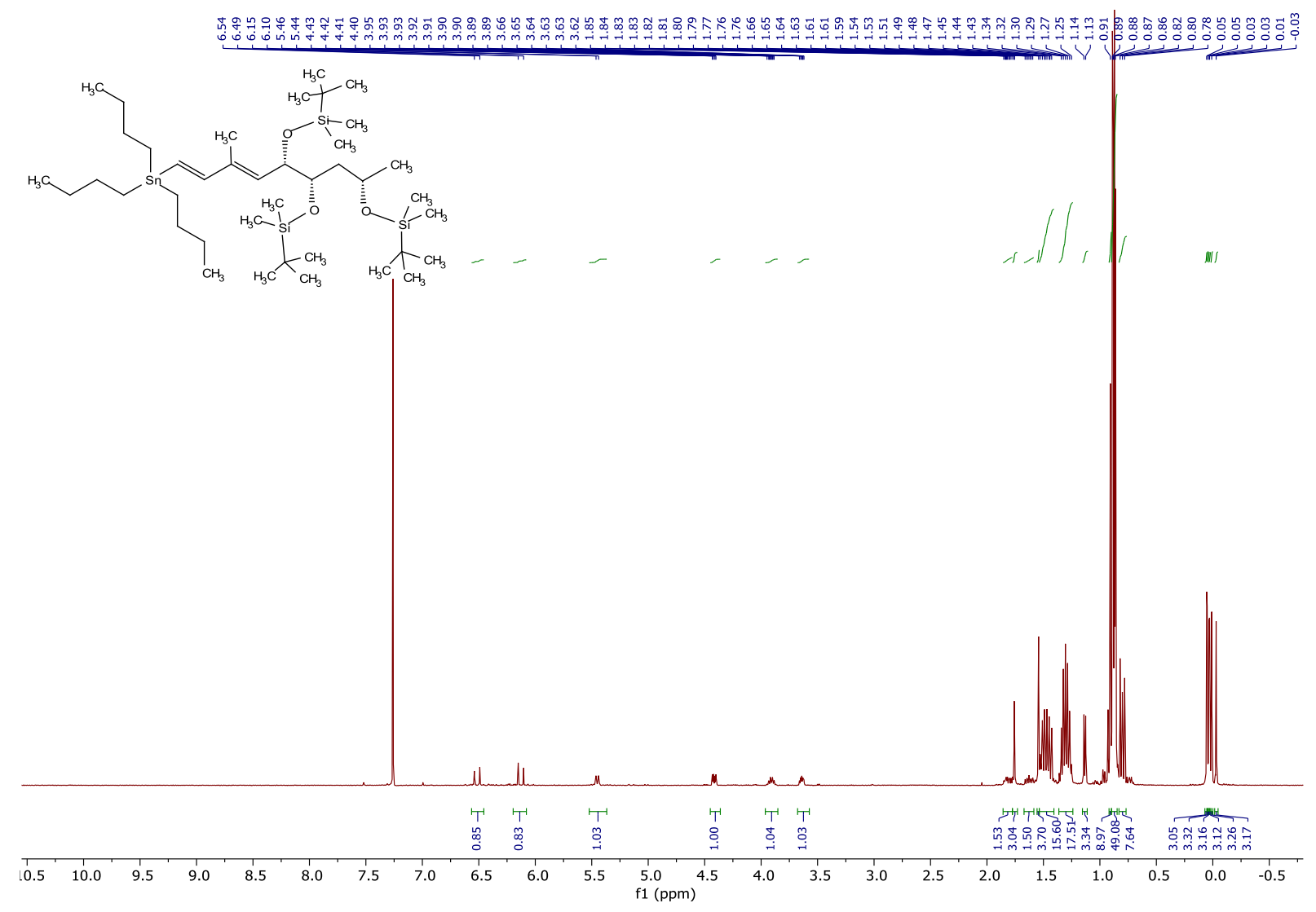



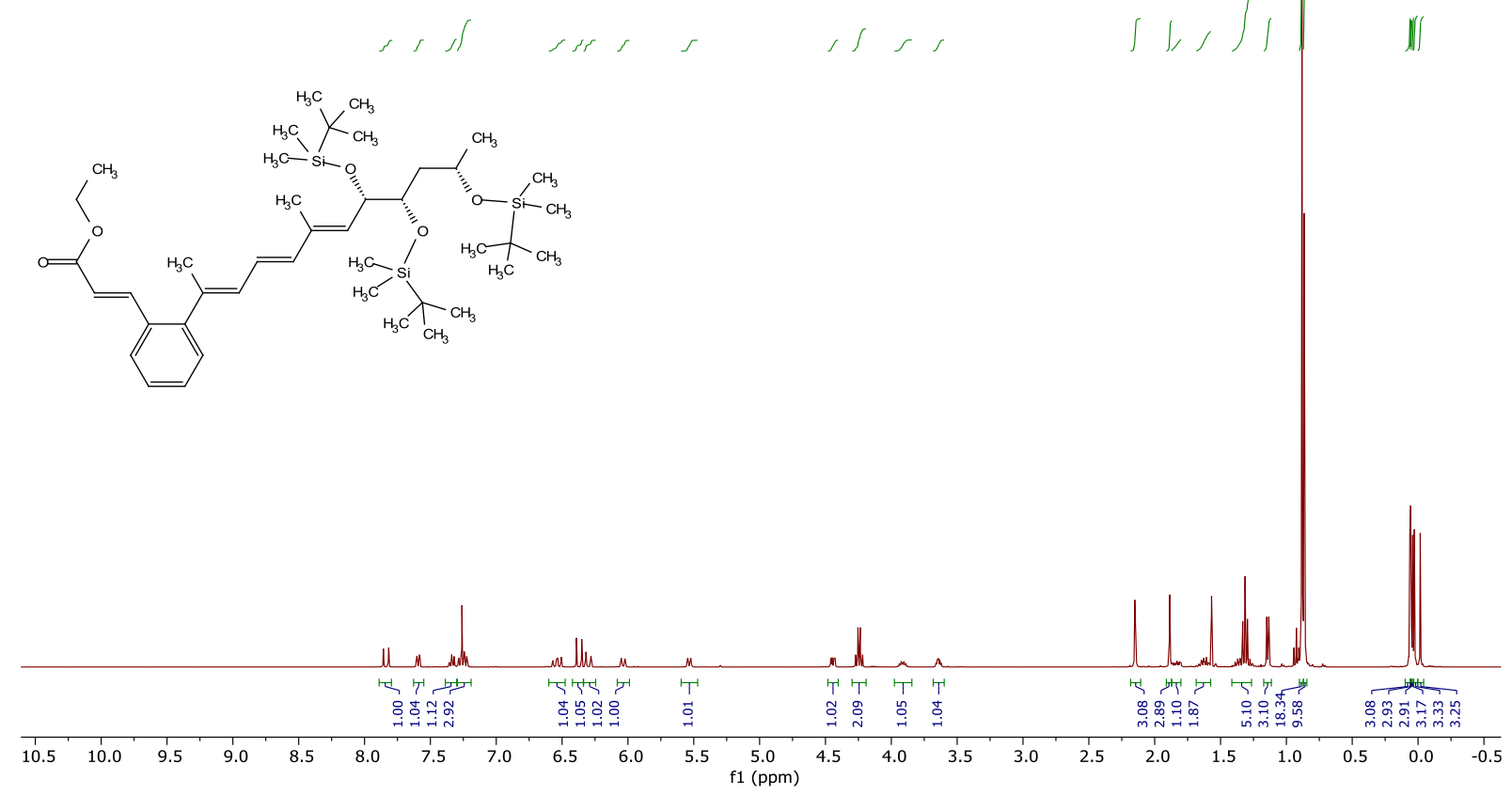

${ }^{13}$ C-NMR of compound $\mathbf{S 9}$
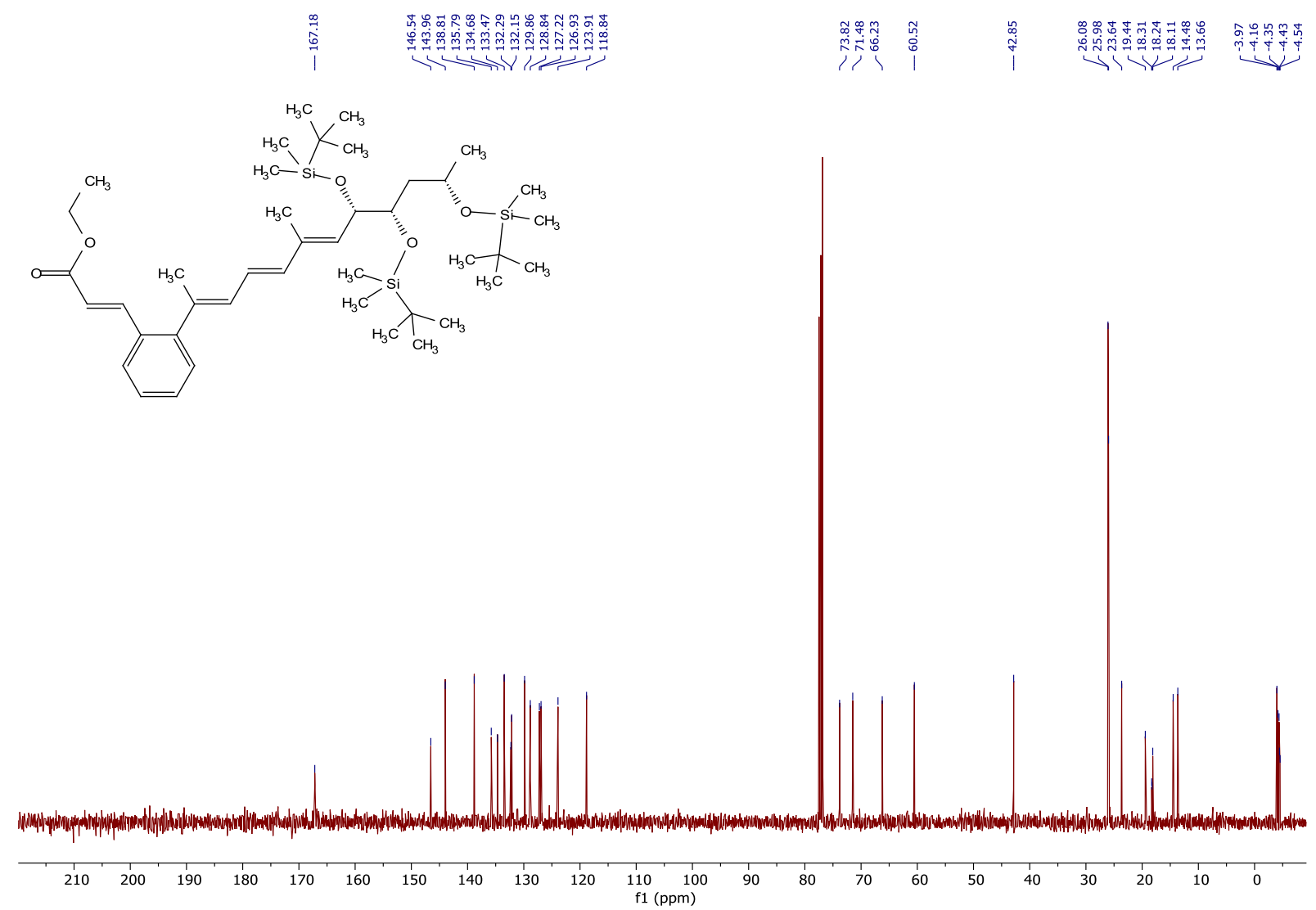


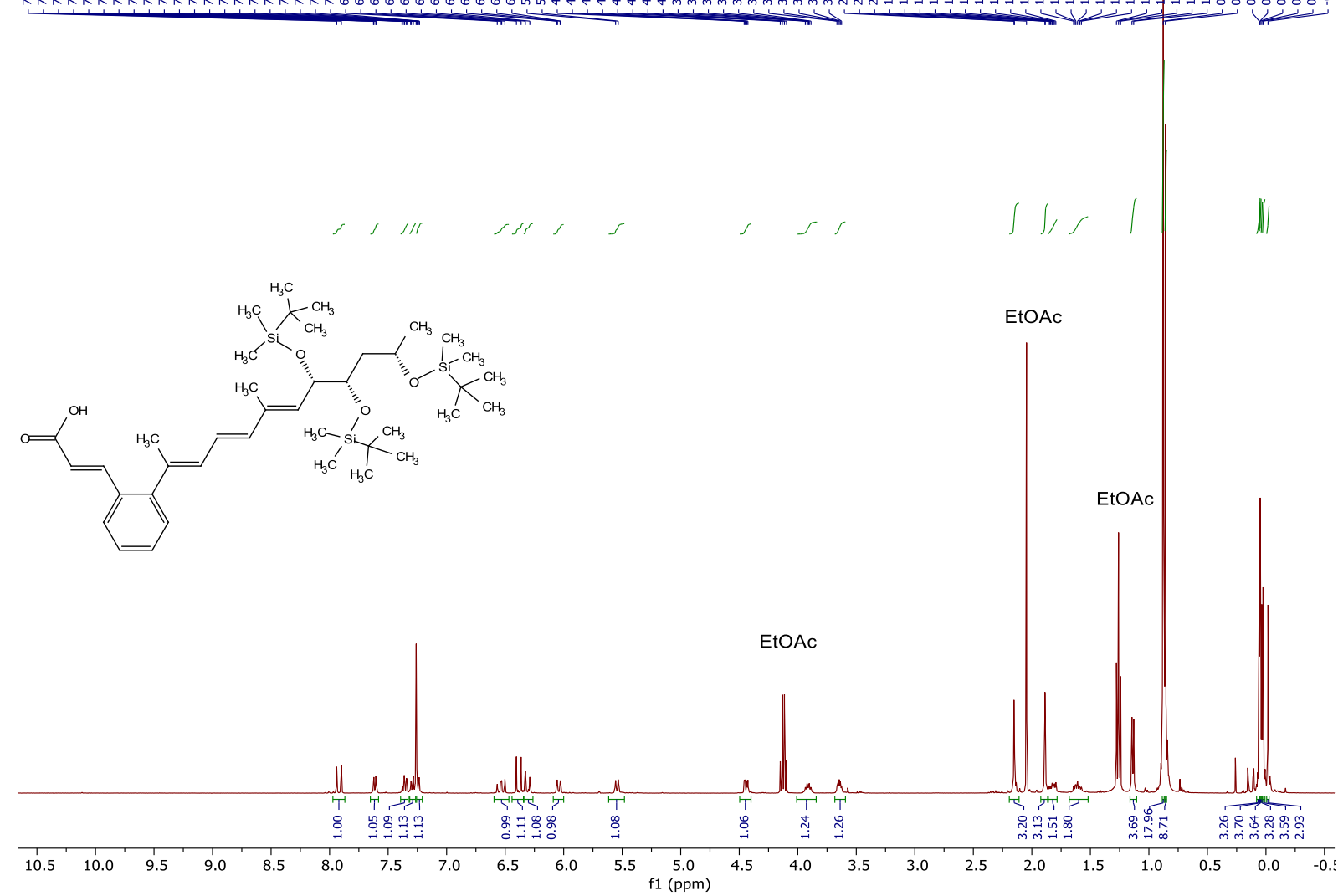

\section{${ }^{13}$ C-NMR of compound $\mathbf{4 b}$}

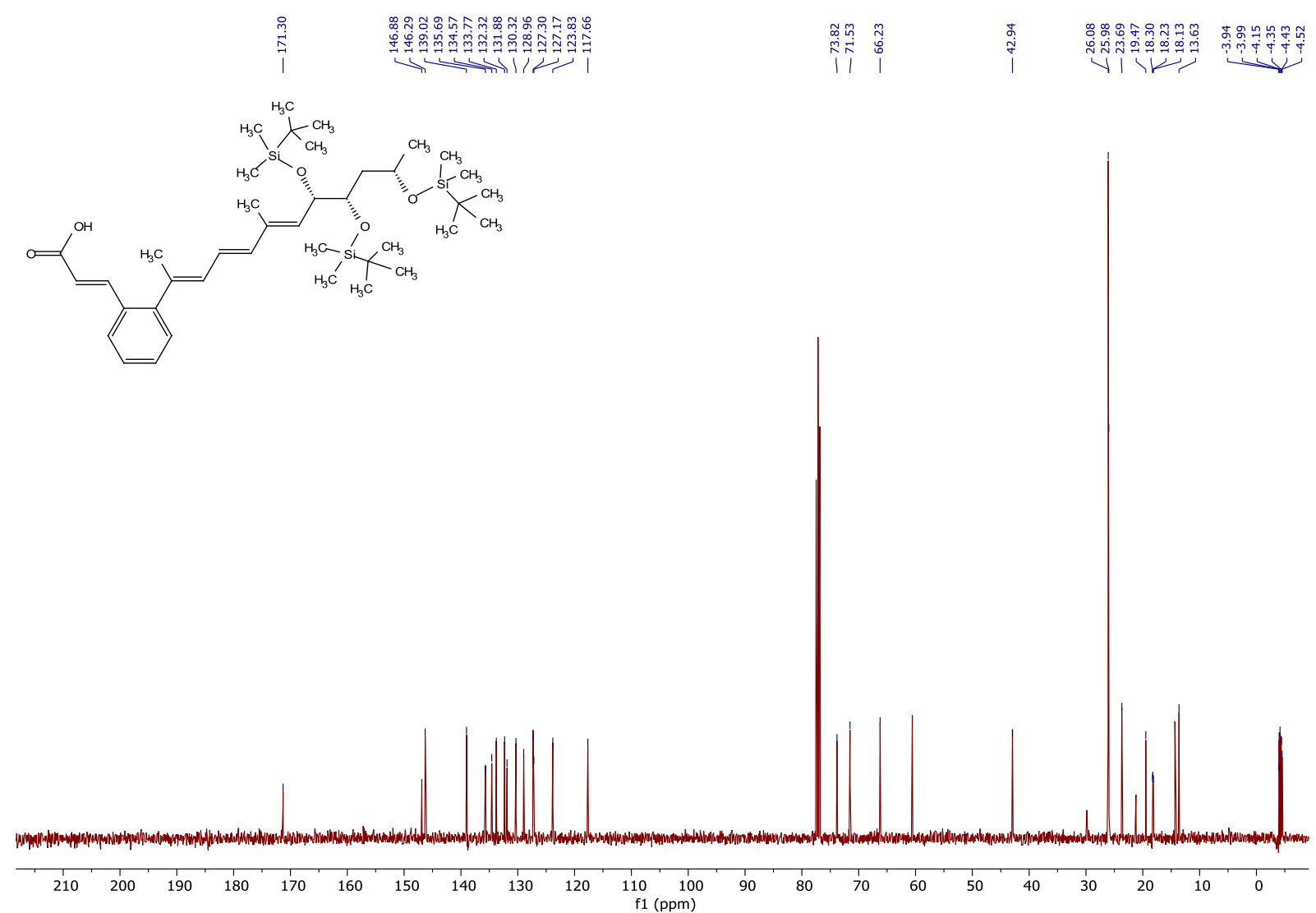


${ }^{1} H$-NMR of compound $\mathbf{S 1 2}$

$\stackrel{2}{i}$

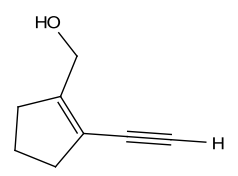

品

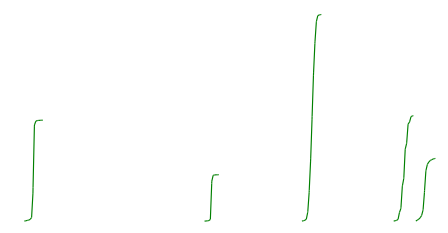

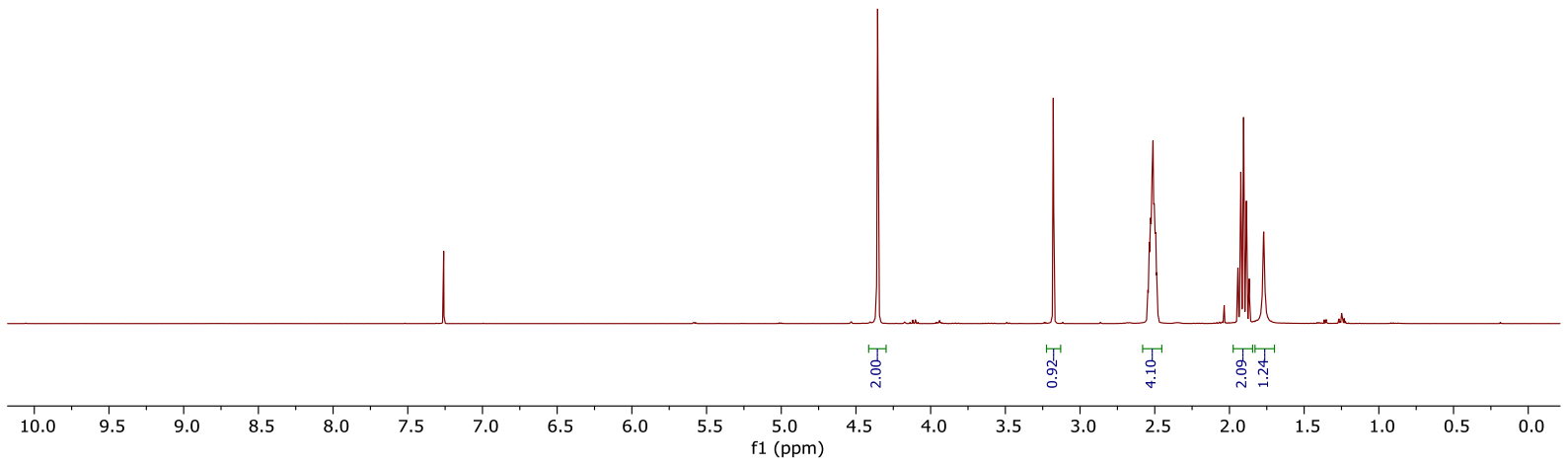

${ }^{13} \mathrm{C}$-NMR of compound $\mathbf{S 1 2}$

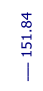

$\stackrel{\circ}{\stackrel{9}{1}}$

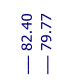

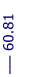

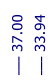

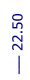
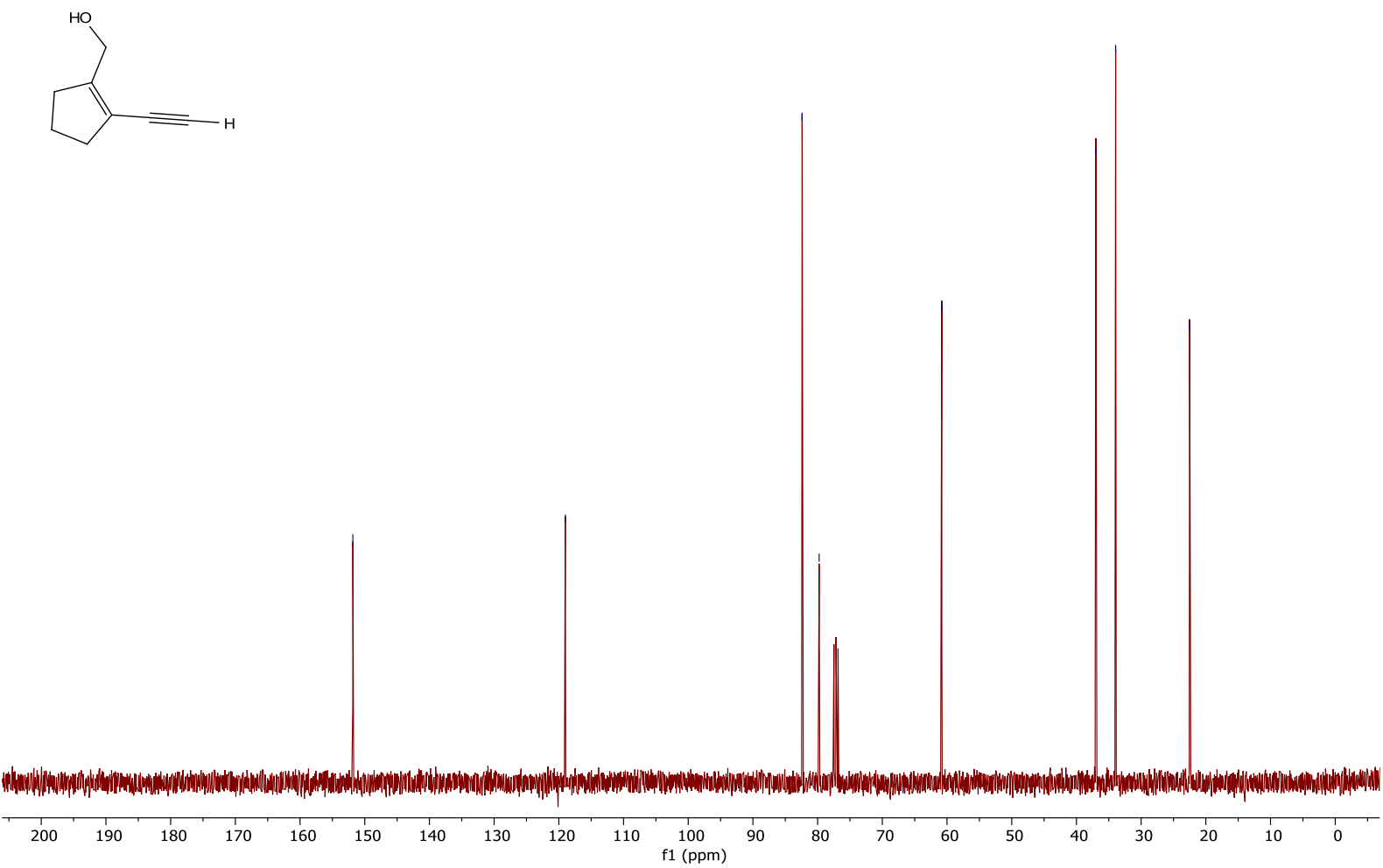

56 
${ }^{1}$ H-NMR of compound 17

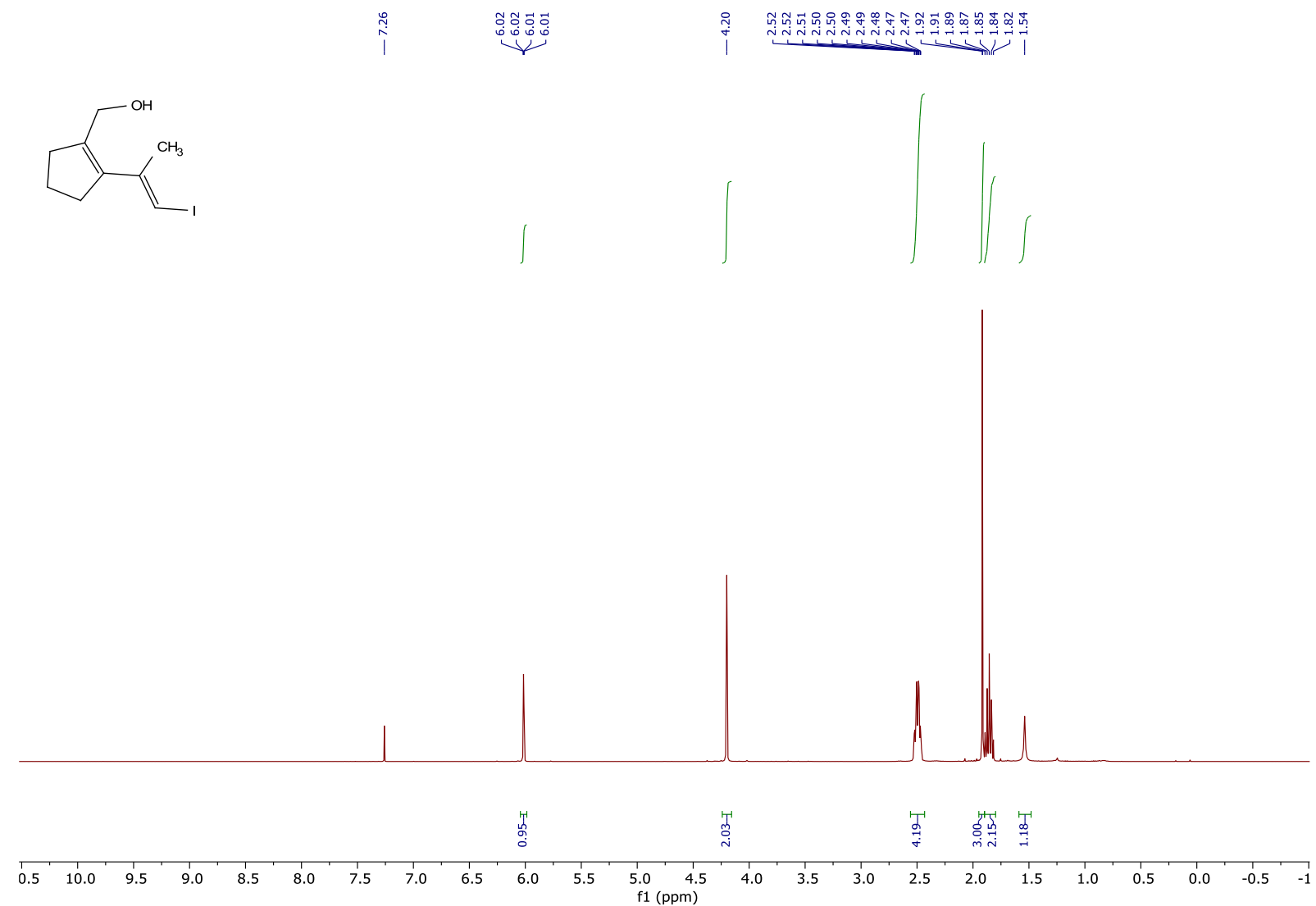

${ }^{13}$ C-NMR of compound 17

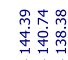

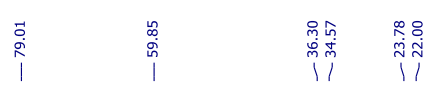
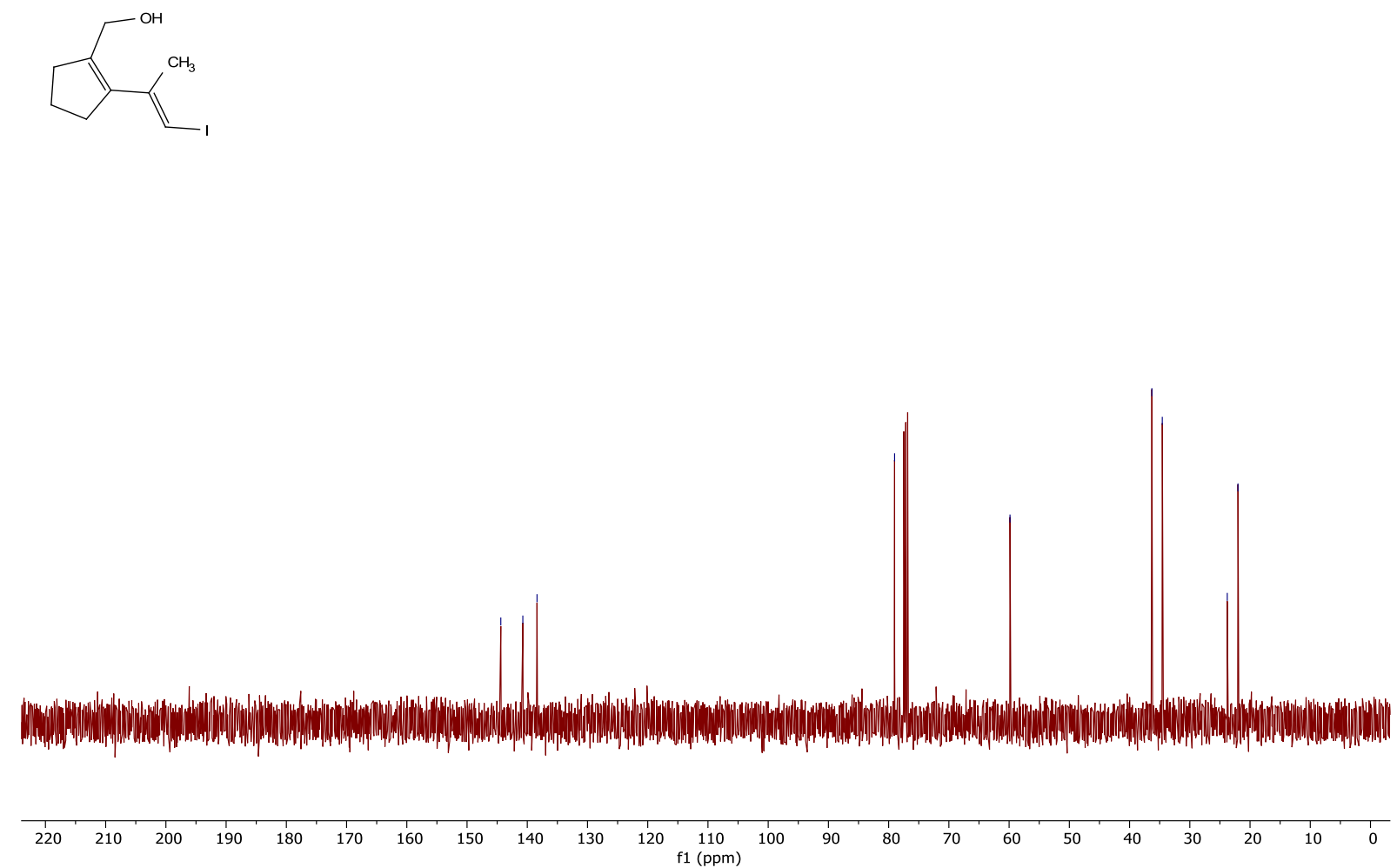
${ }^{1} H$-NMR of compound 13 (crude)

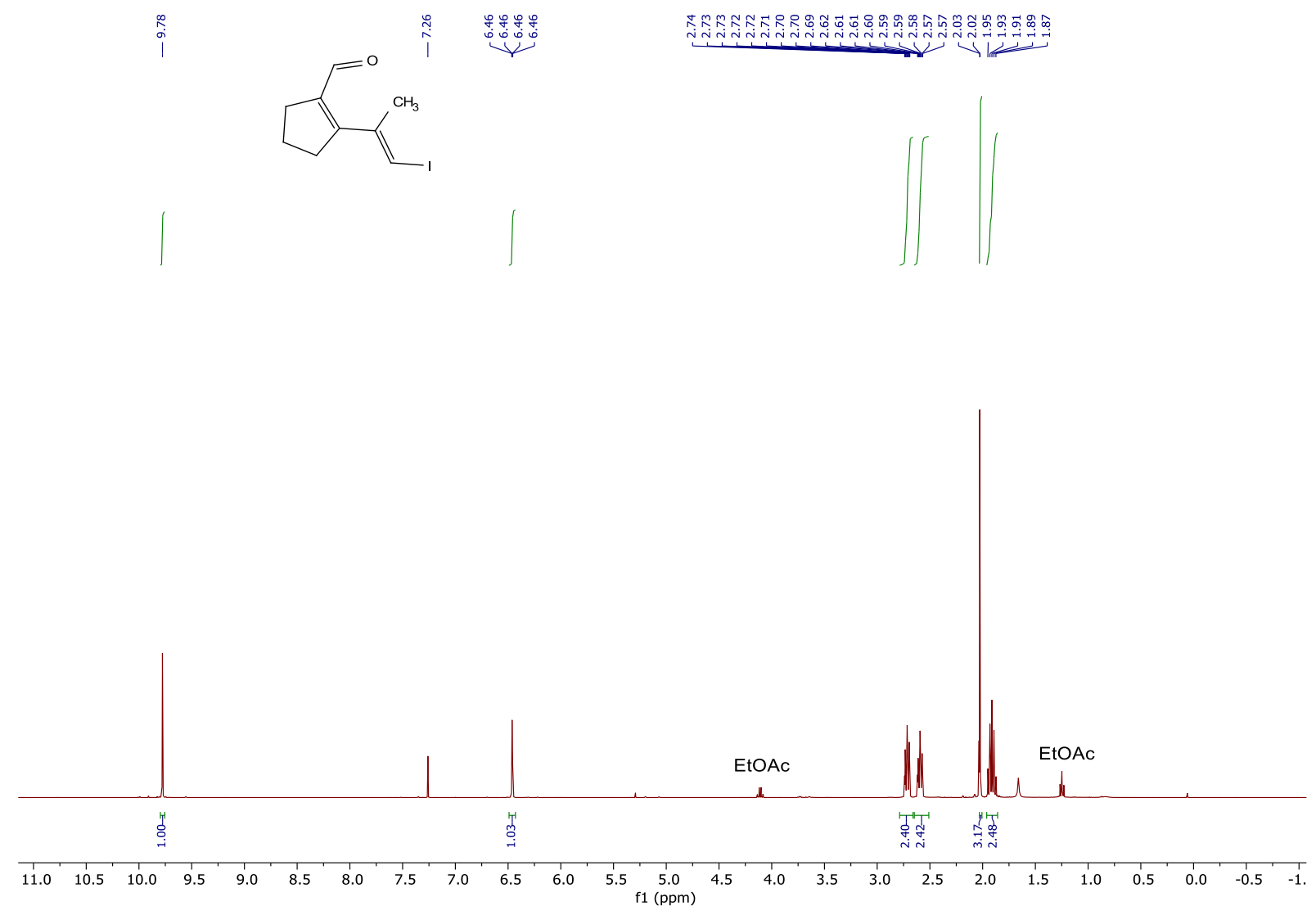

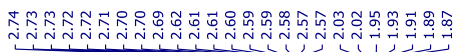

${ }^{13}$ C-NMR of compound $\mathbf{S 1 3}$ (crude)

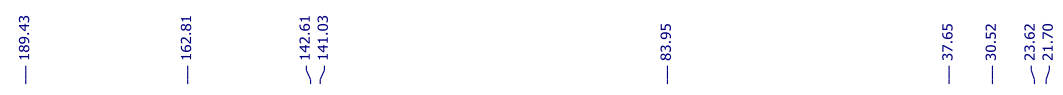
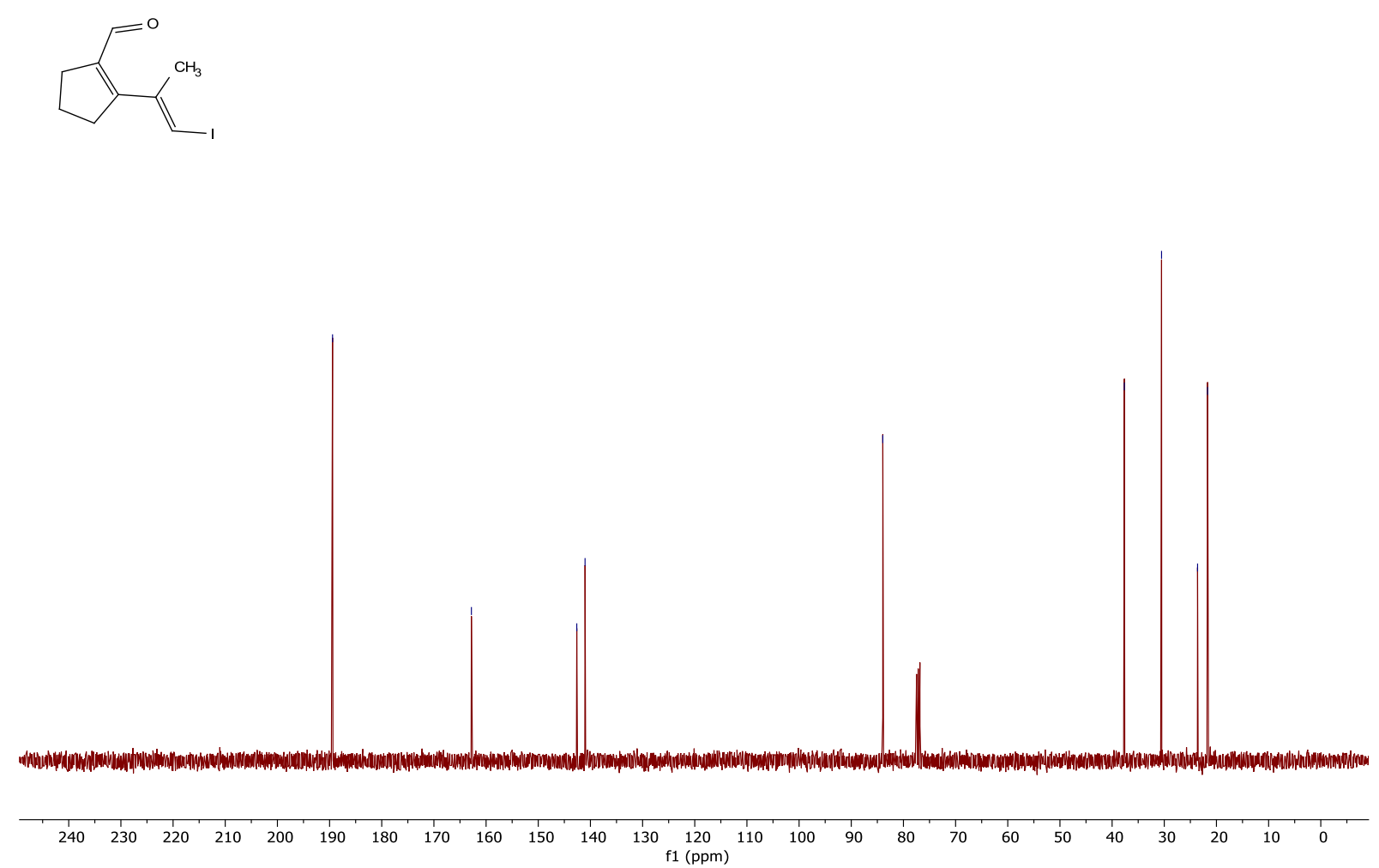
${ }^{1} \mathrm{H}$-NMR of compound 18

il i i
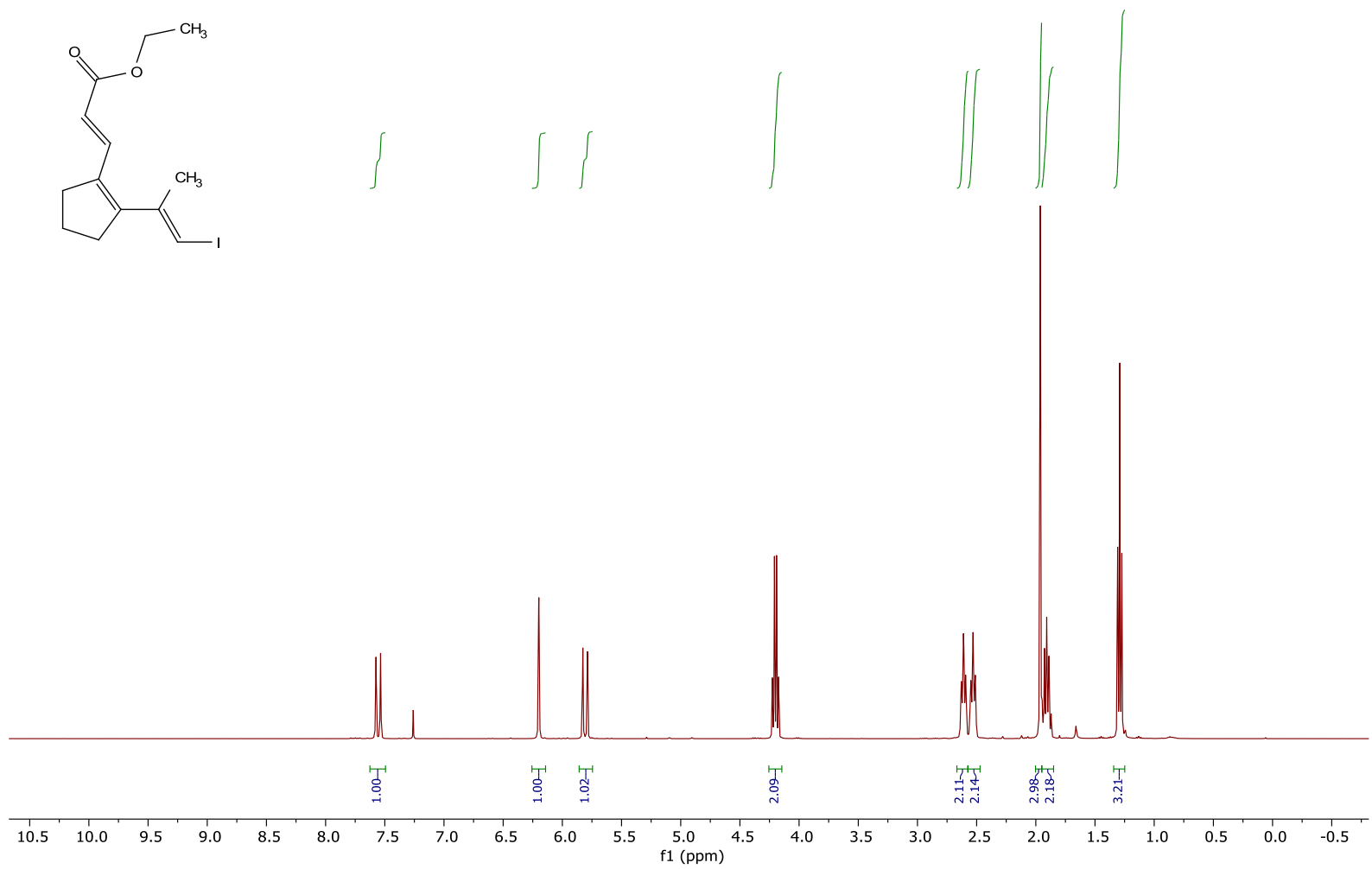

${ }^{13}$ C -NMR of compound 18

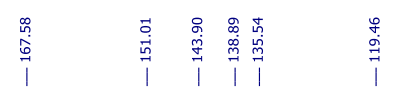

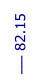

i.
i.
i

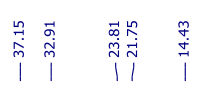
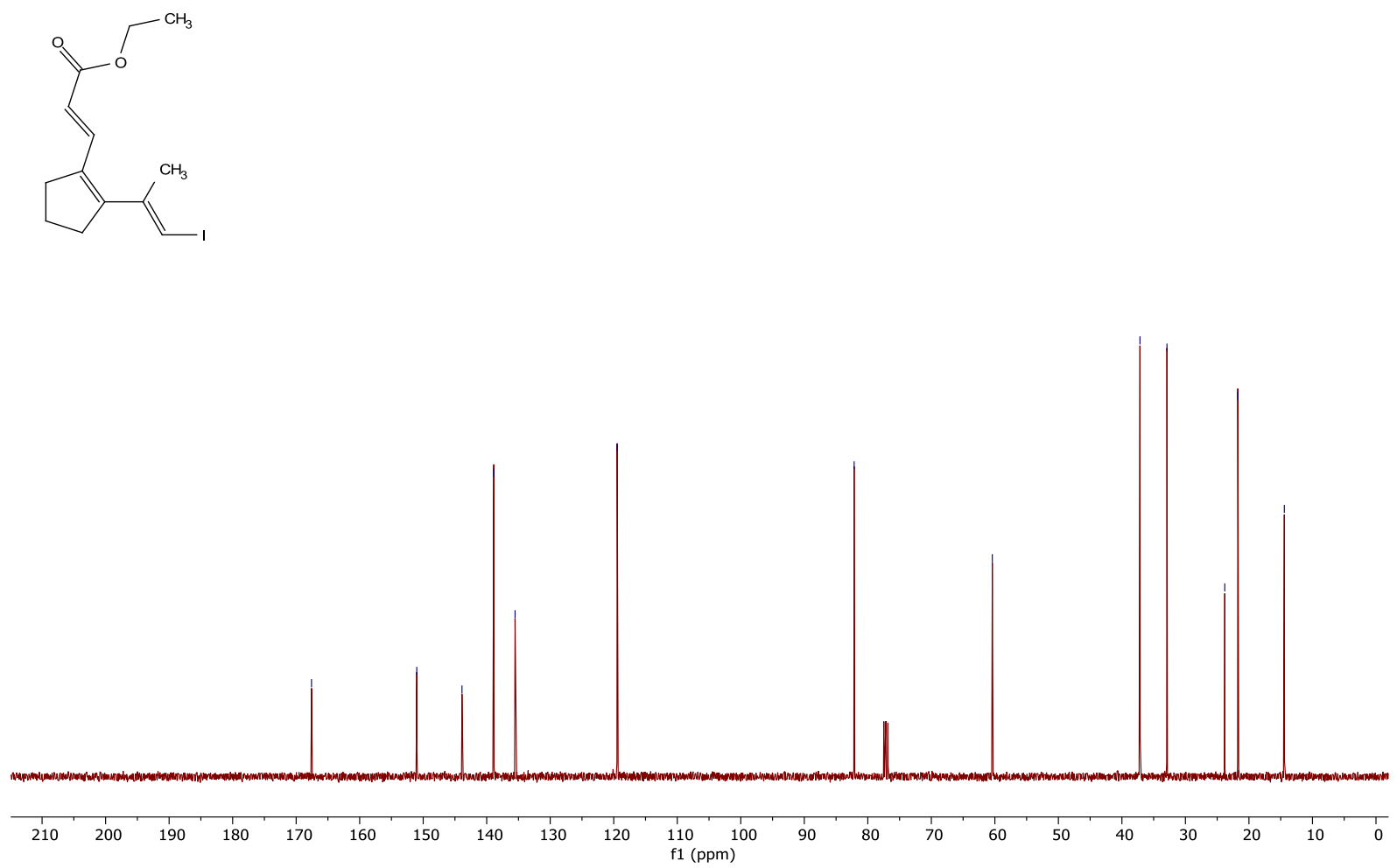

59 


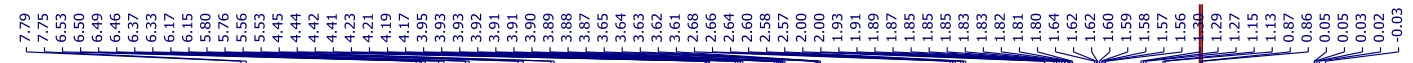
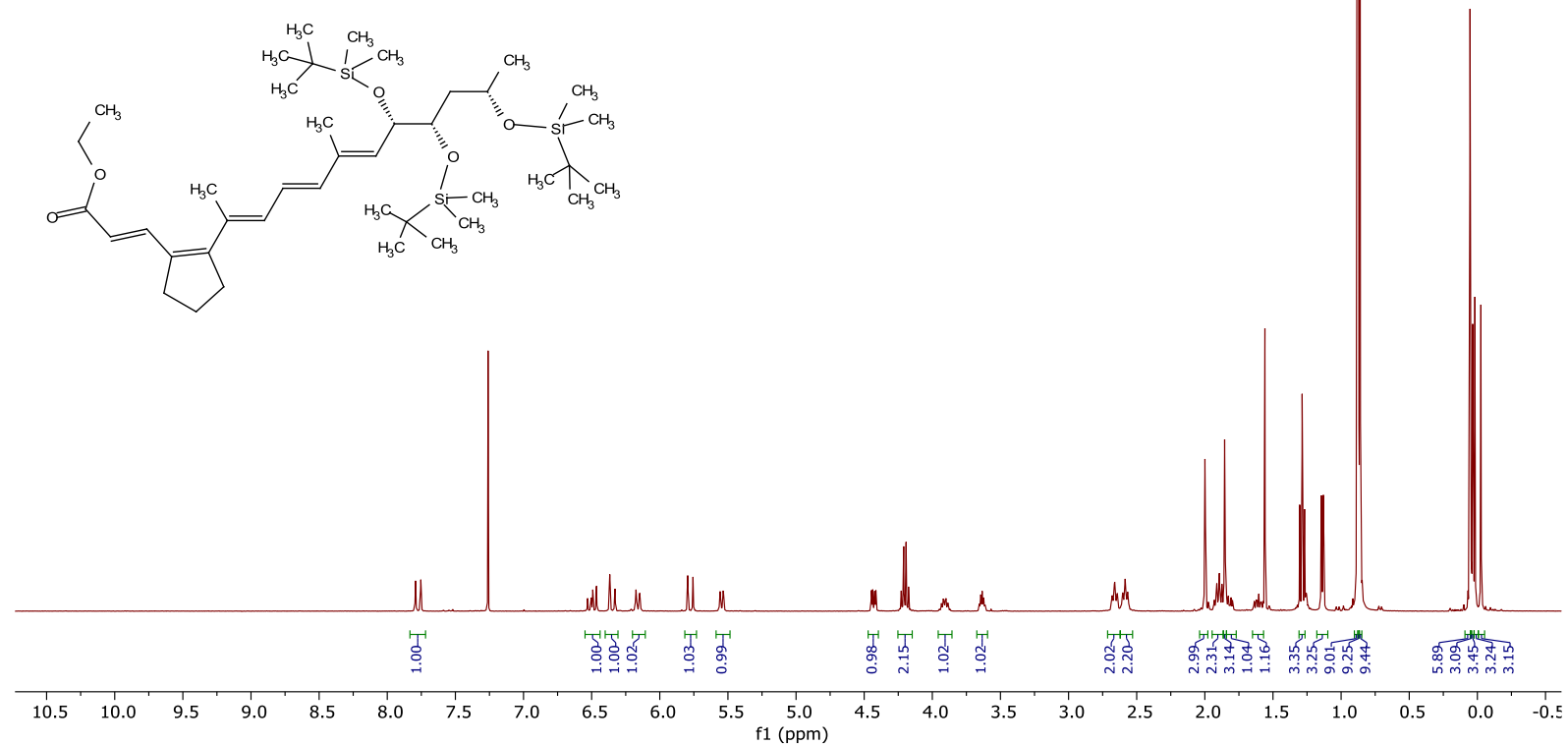

${ }^{13}$ C-NMR of compound $\mathbf{S 1 4}$

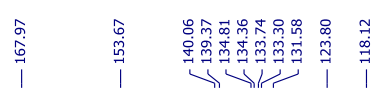

|l
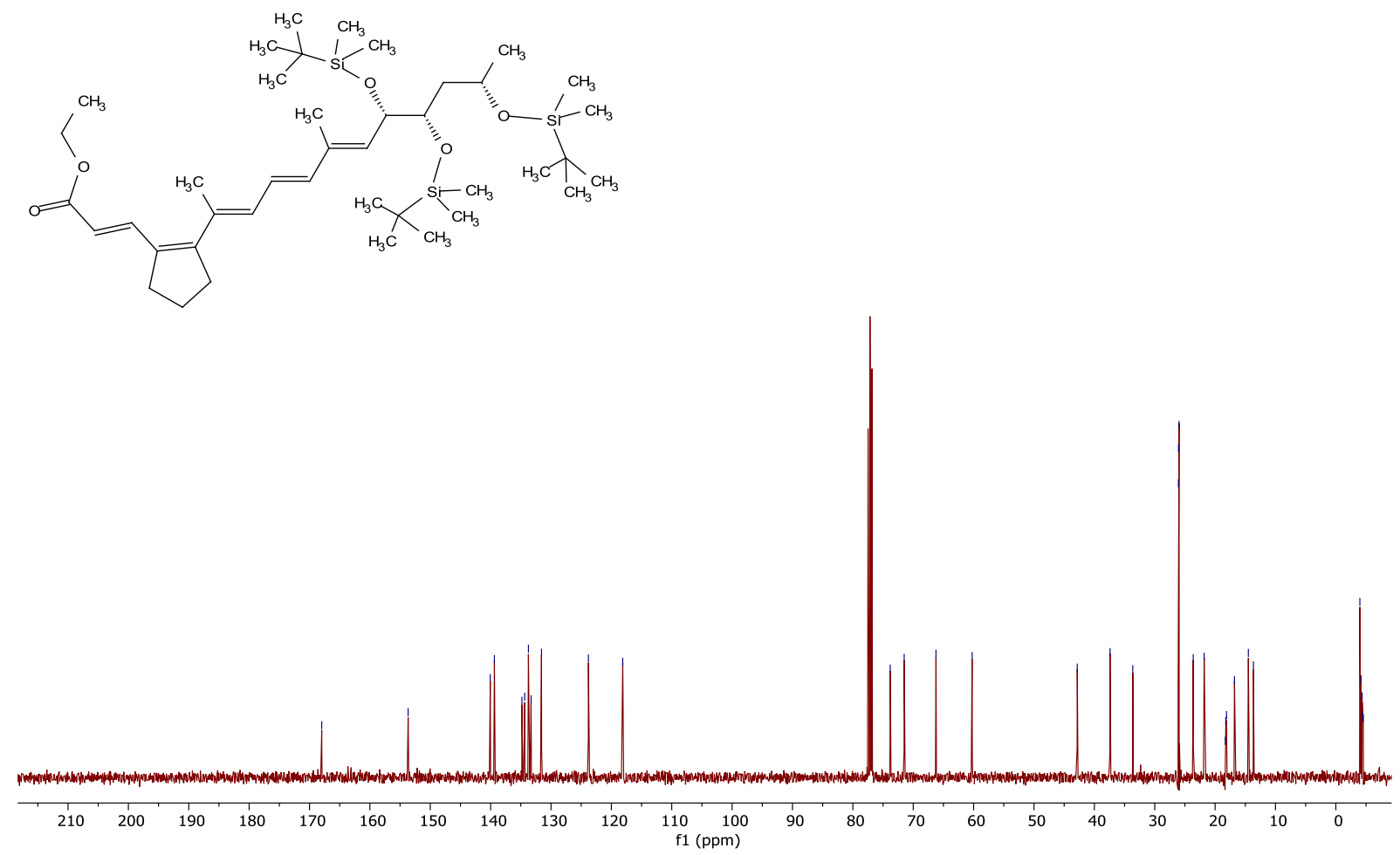


\section{${ }^{1} \mathrm{H}$-NMR of compound $\mathbf{4 c}$}

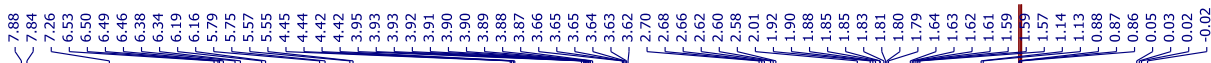
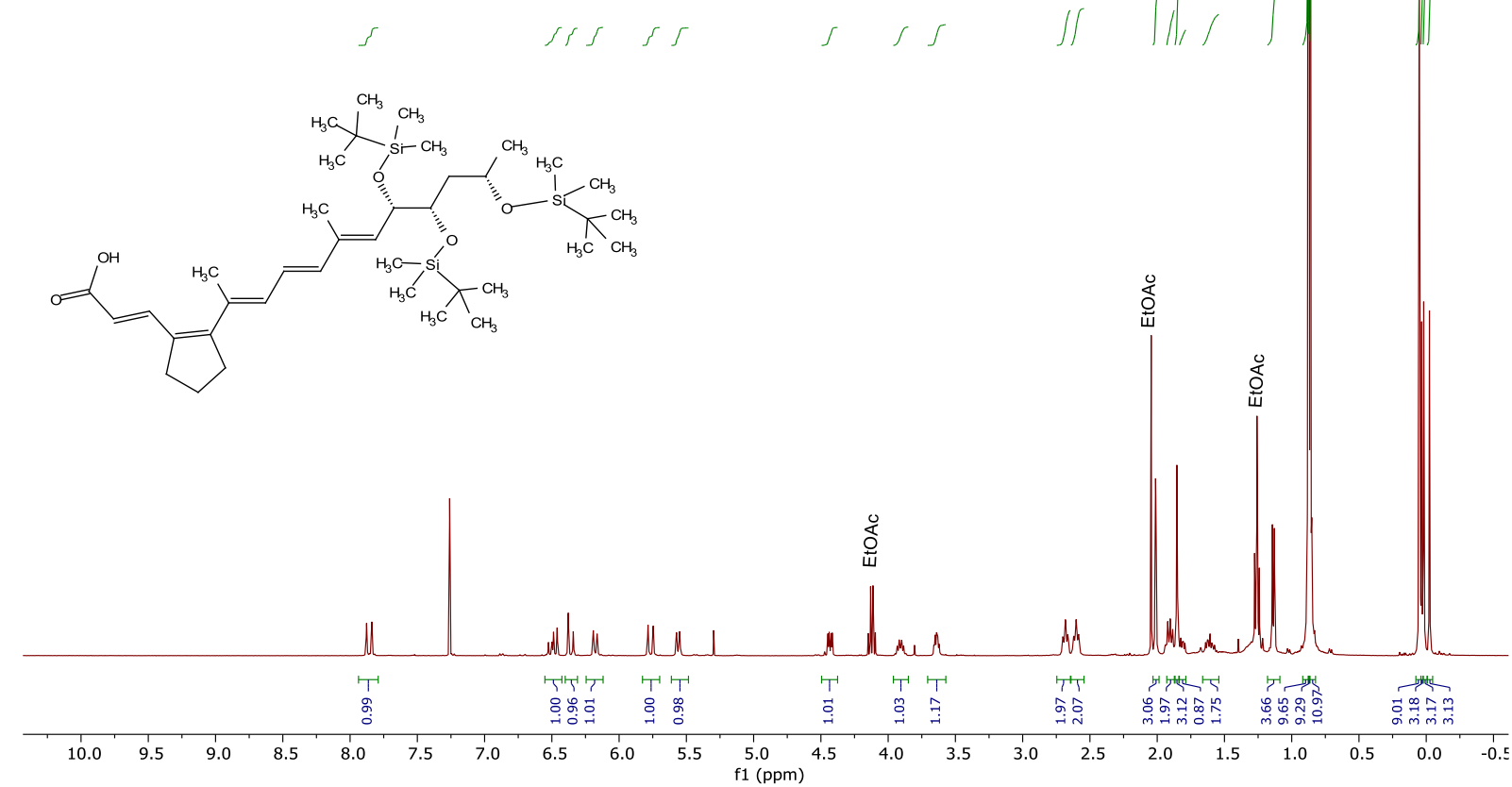

${ }^{13} \mathrm{C}-\mathrm{NMR}$ of compound $\mathbf{4 c}$

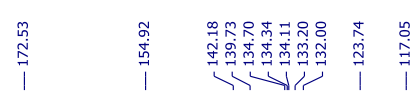

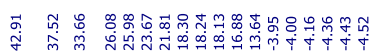
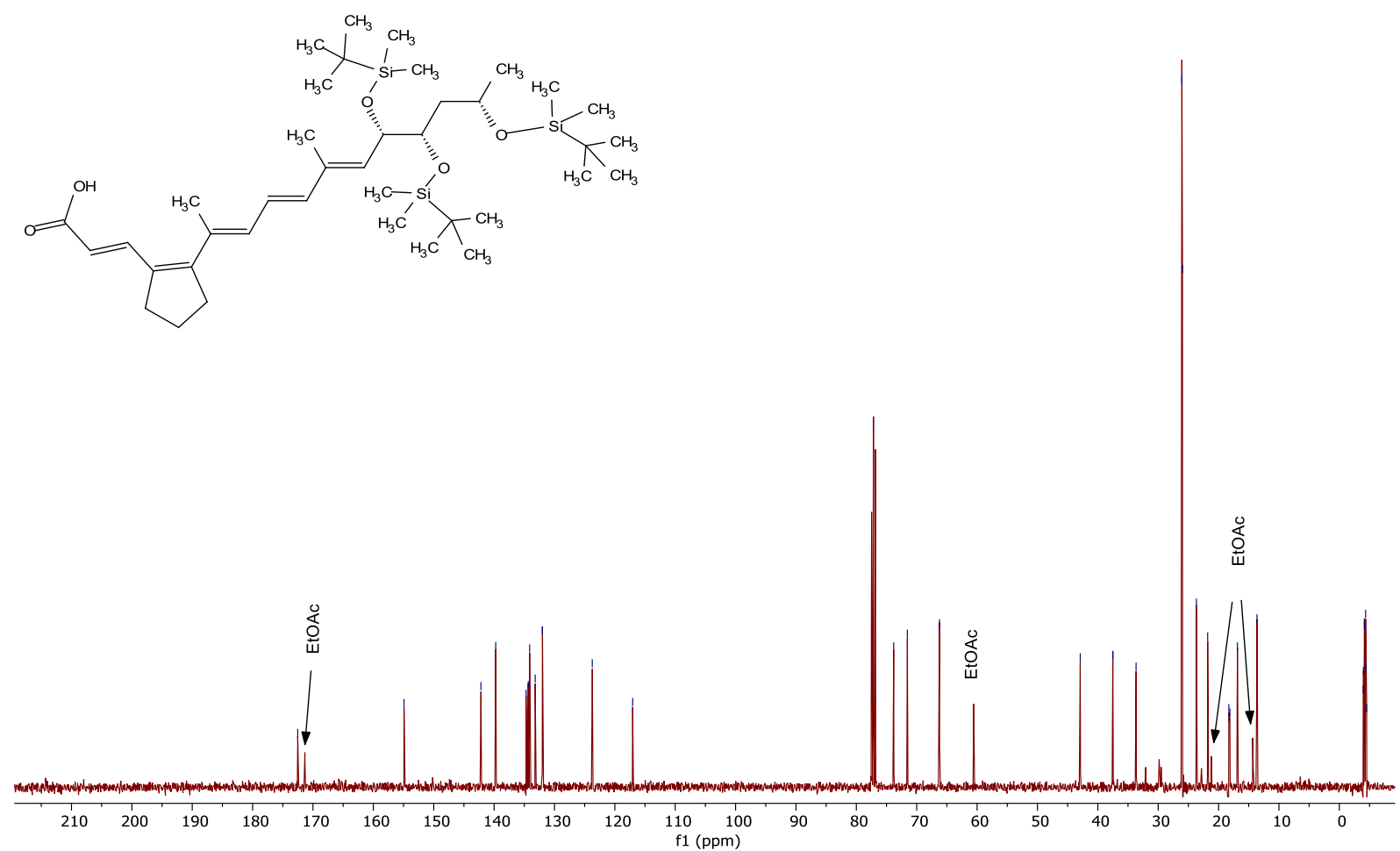

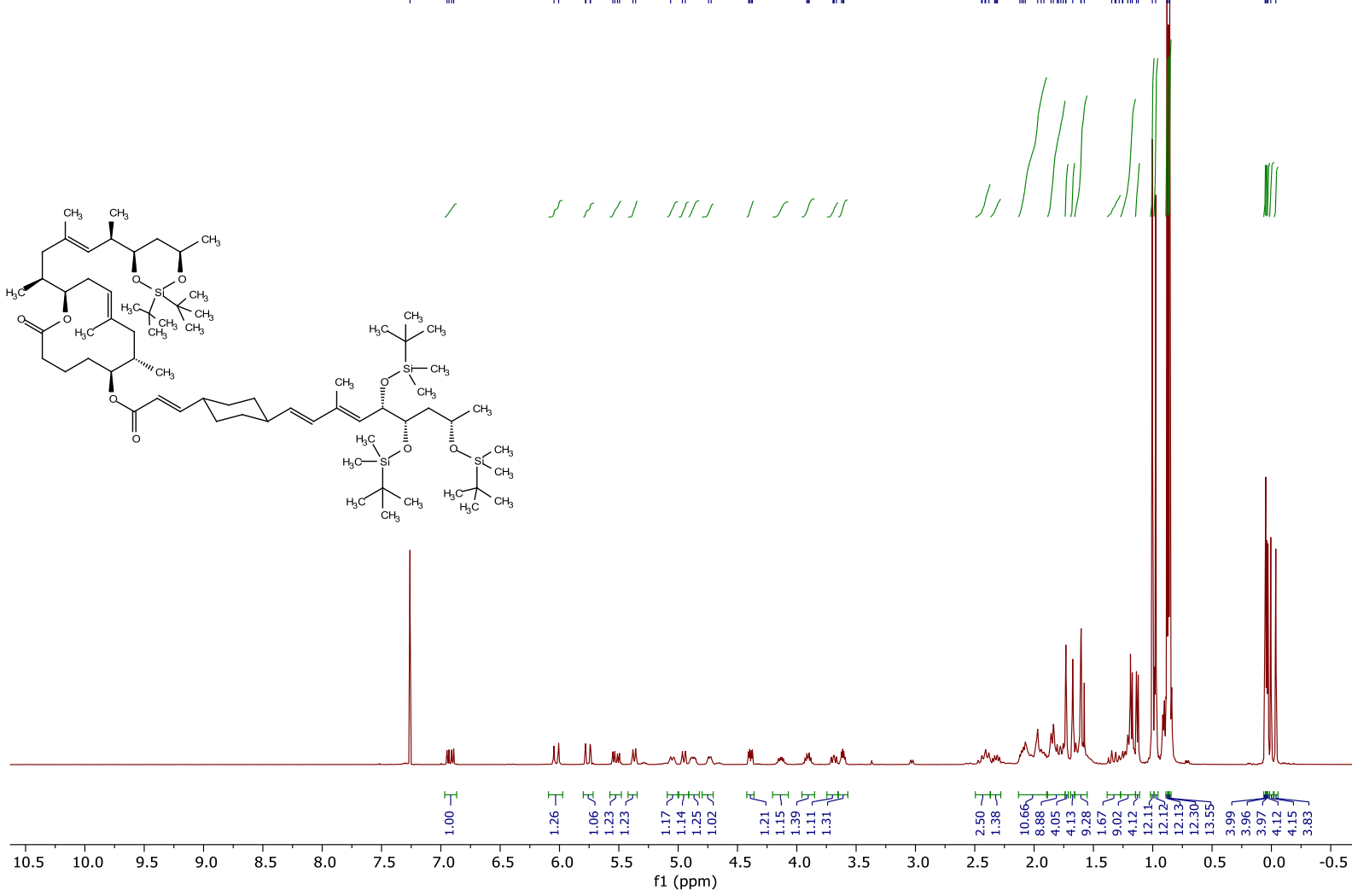

\section{${ }^{13}$ C-NMR of compound $\mathbf{S 1 5}$}

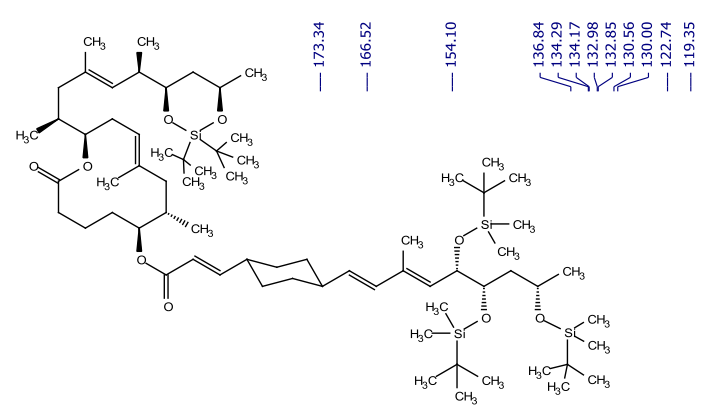

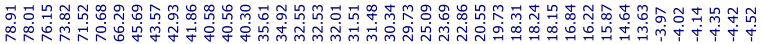




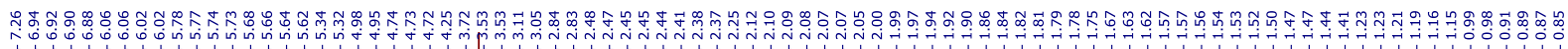

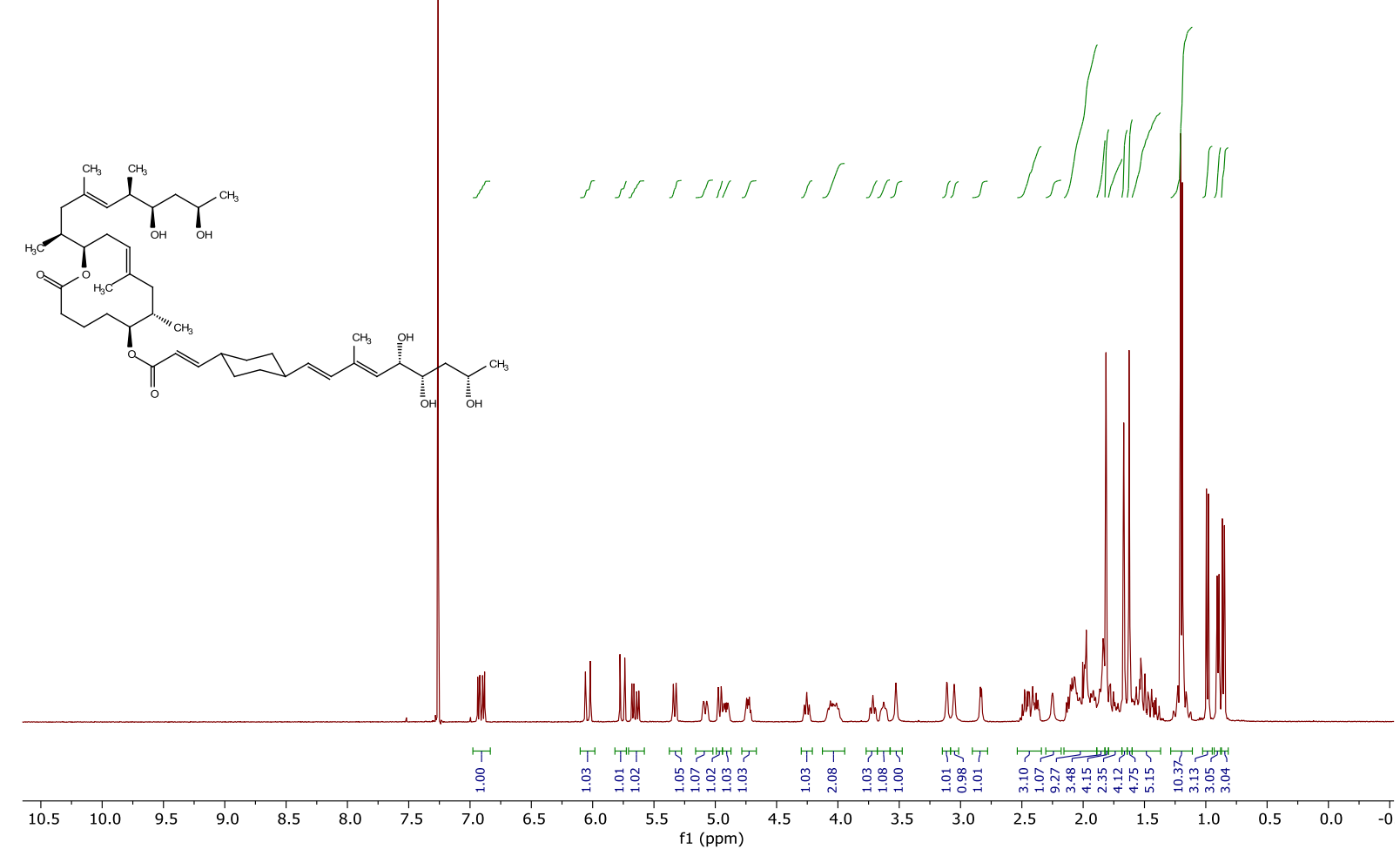

${ }^{13} C$-NMR of compound $2 a$

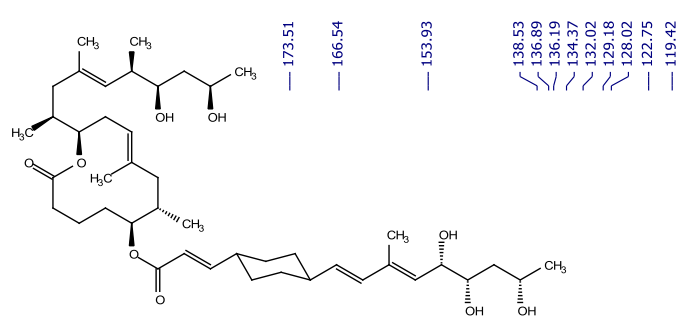

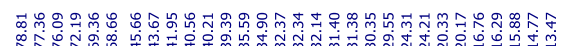

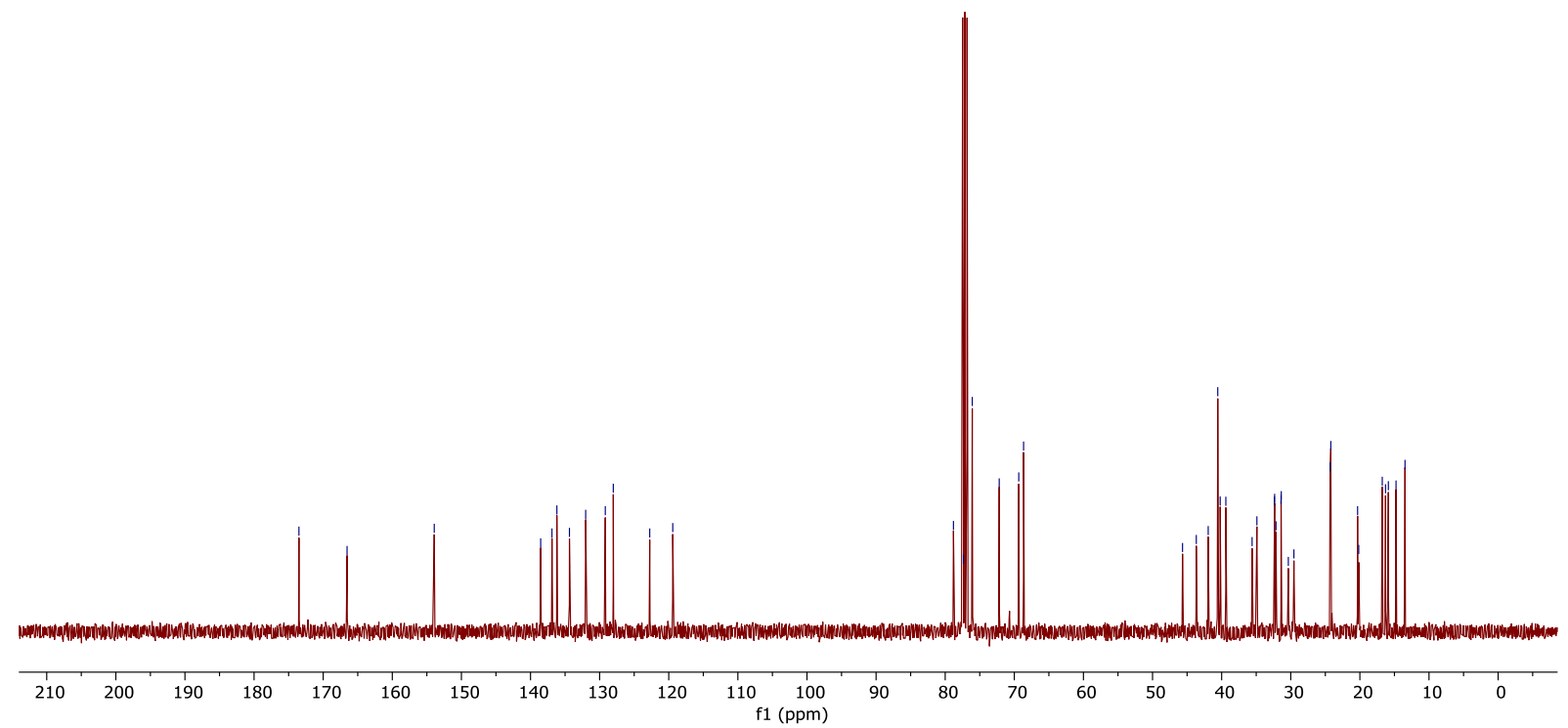




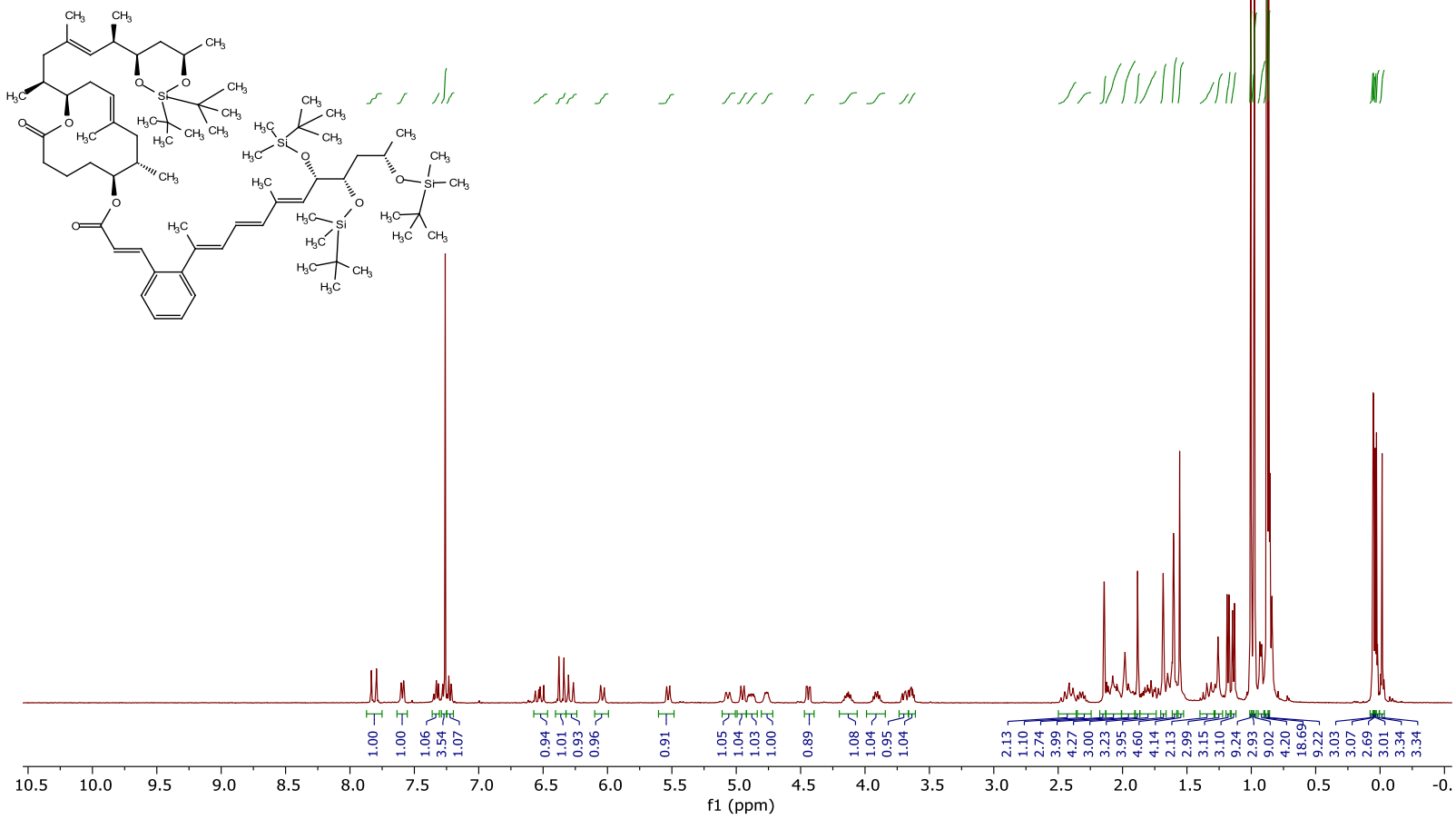

\section{${ }^{13}$ C-NMR of compound $\mathbf{S 1 6}$}

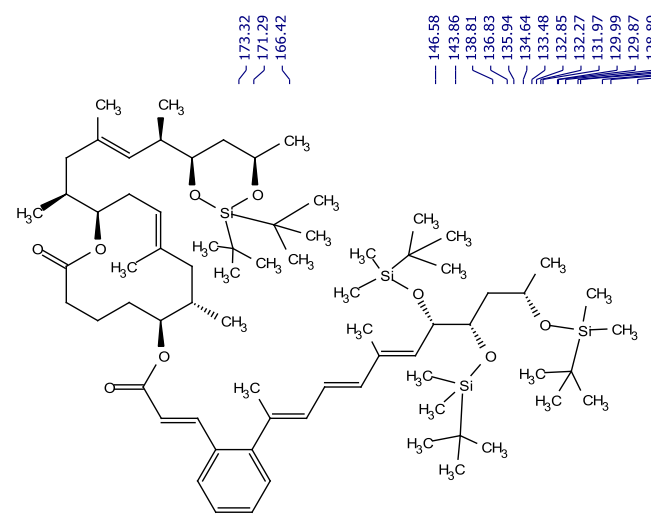

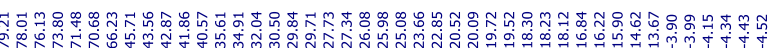

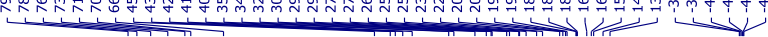

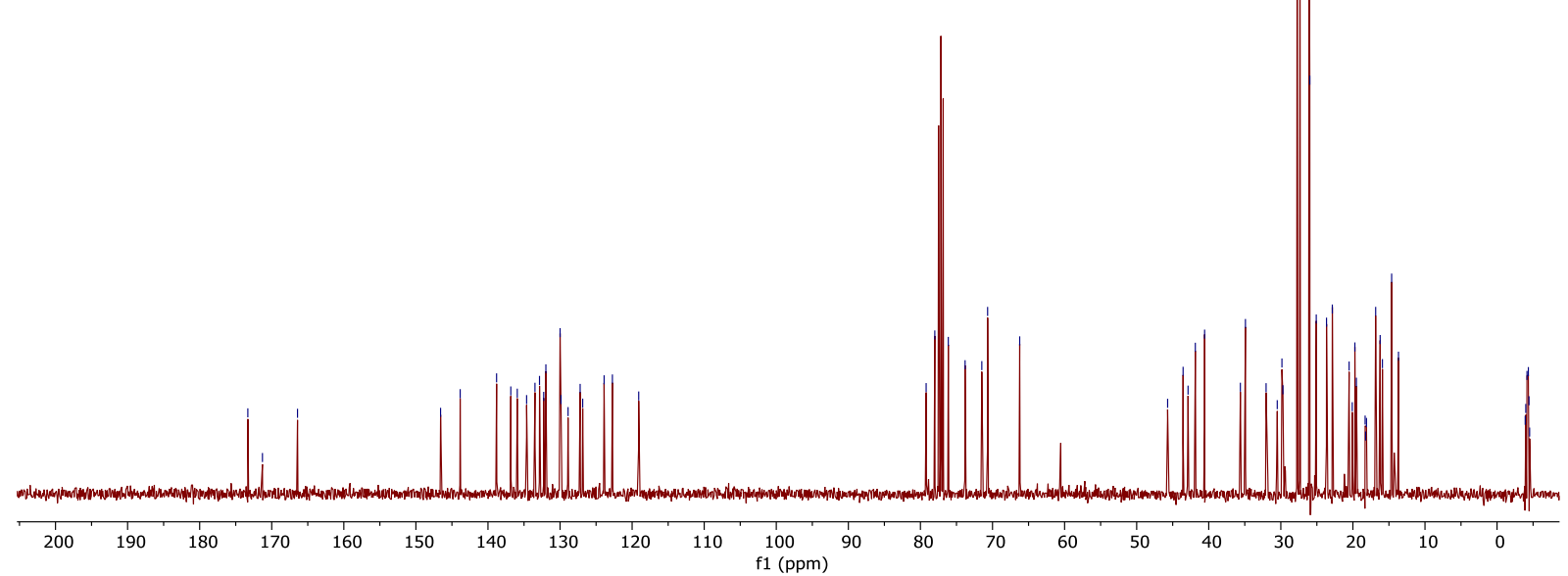




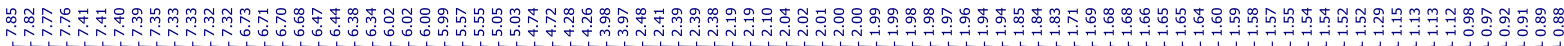

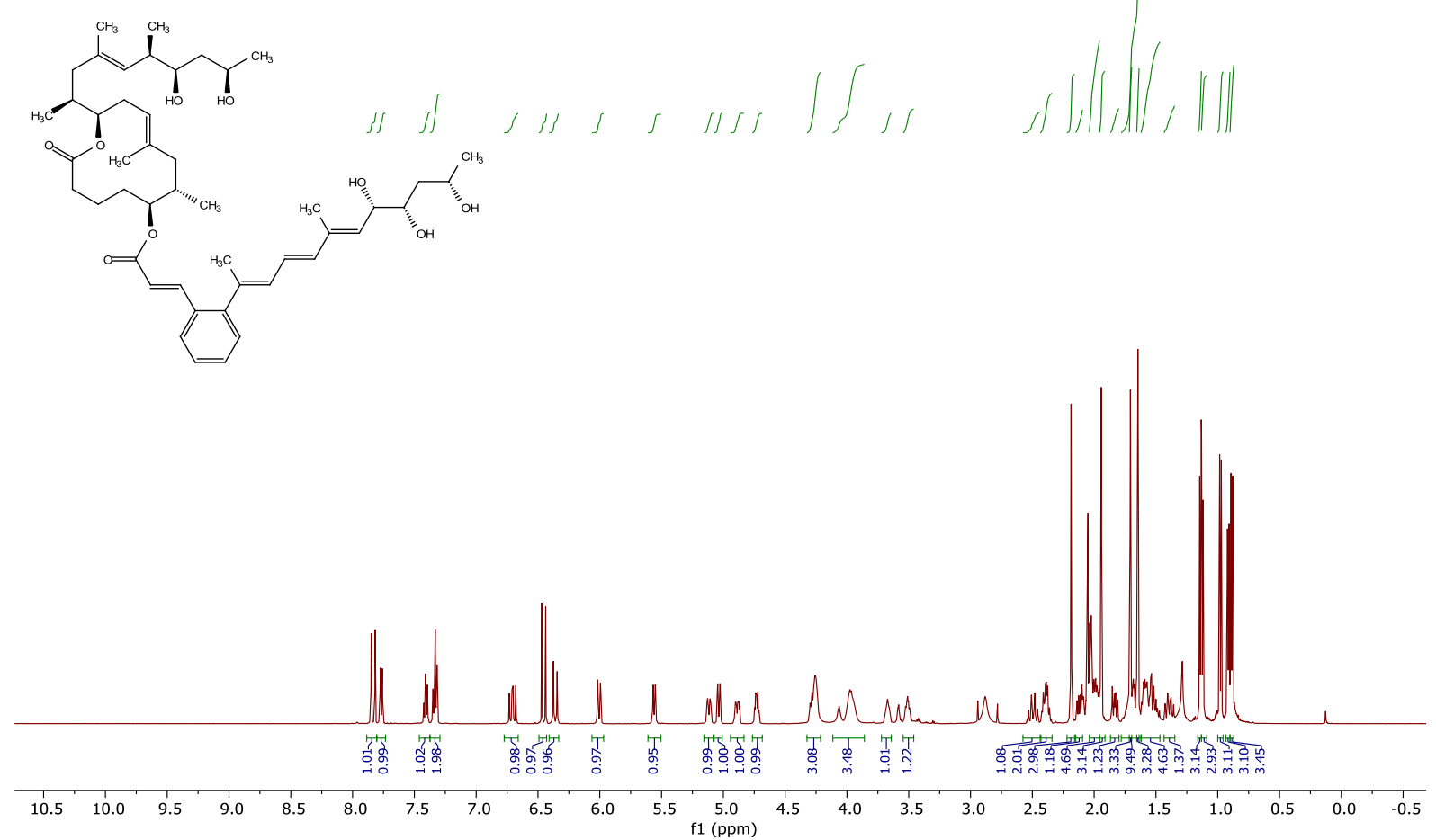

\section{${ }^{13} C$-NMR of compound $\mathbf{2 b}$}

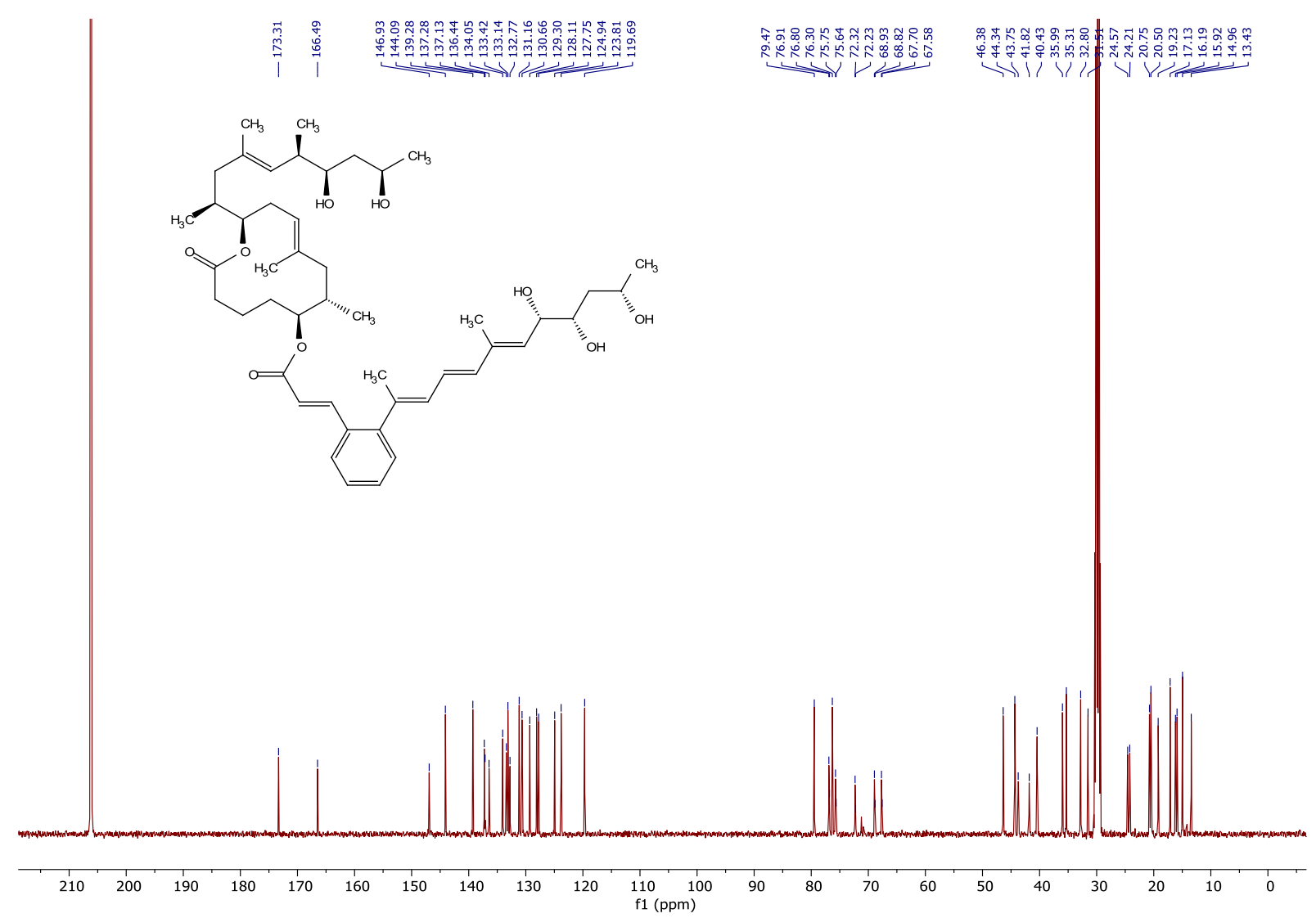




\section{${ }^{1} H$-NMR of compound $\mathbf{S 1 7}$}

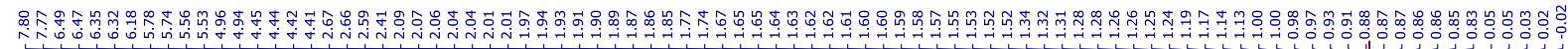

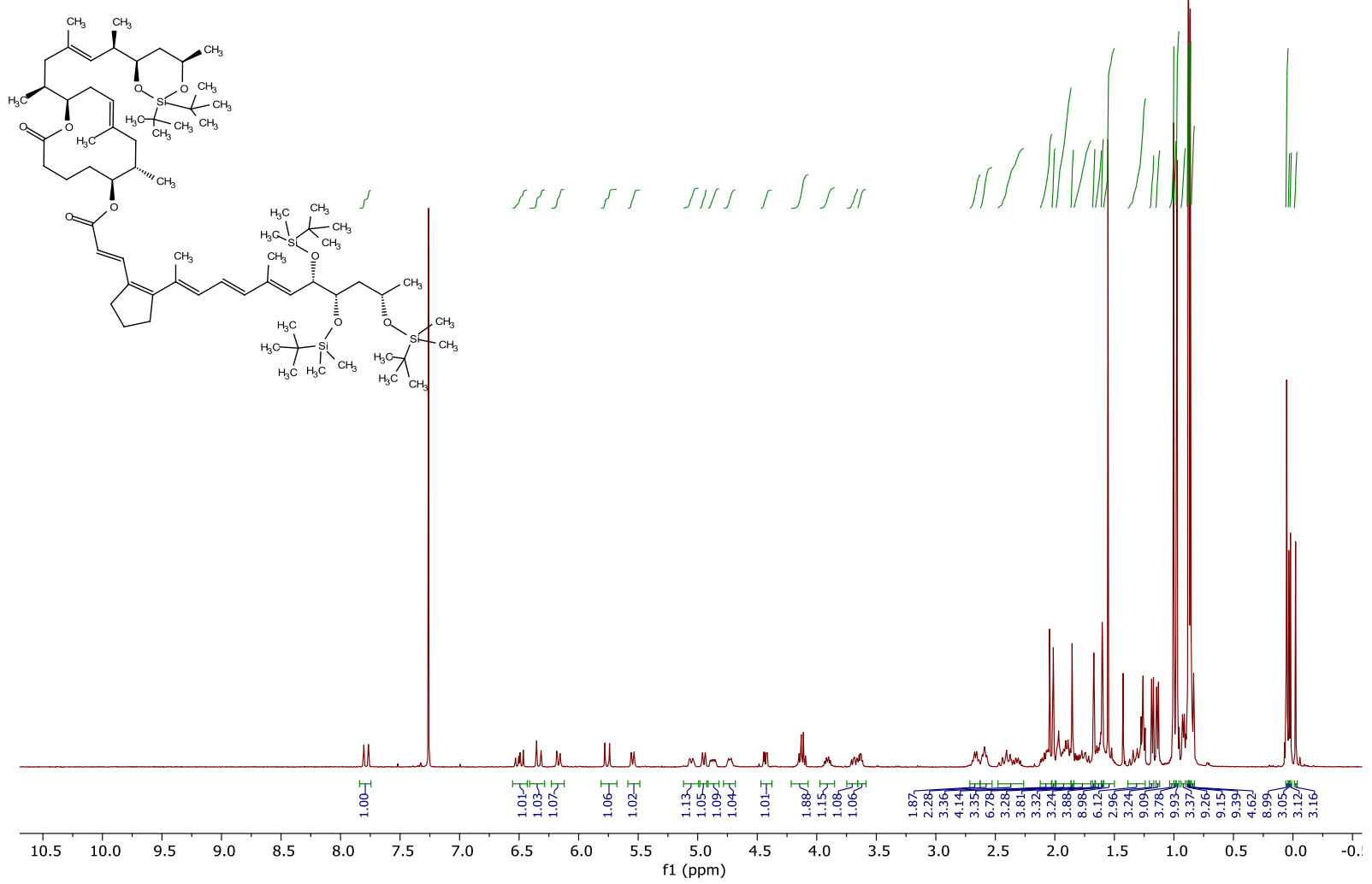

${ }^{13} \mathrm{C}$-NMR of compound $\mathrm{S17}$

|

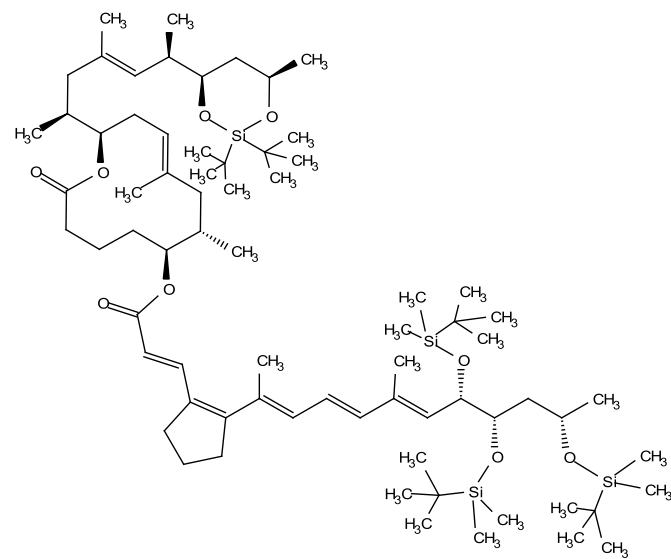

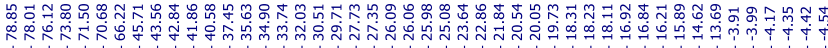

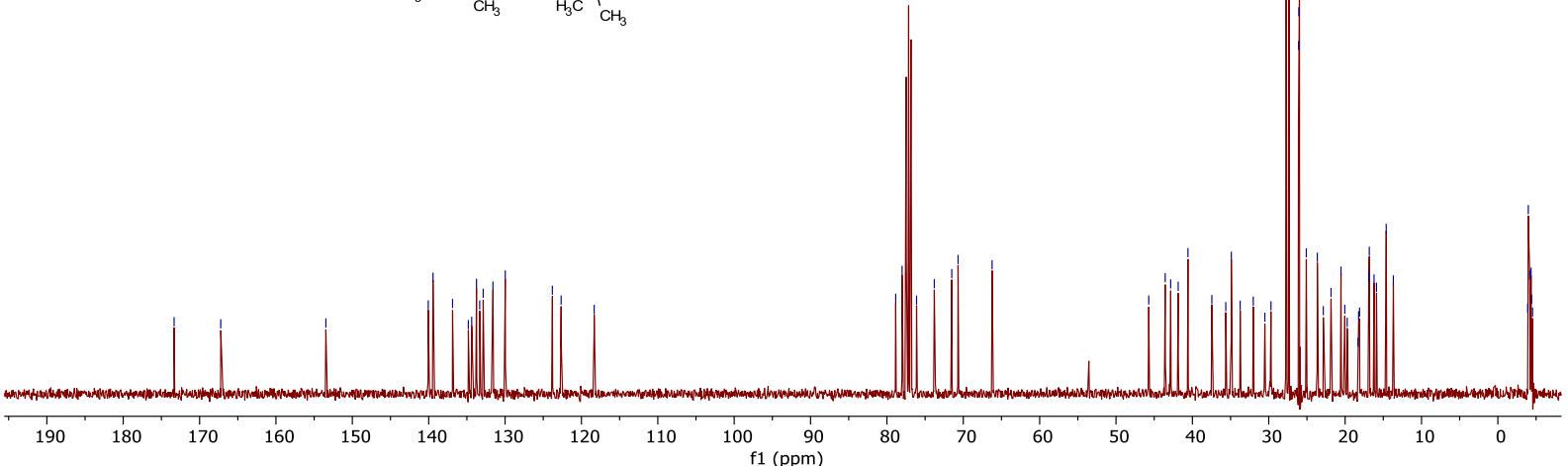




\section{${ }^{1} H$-NMR of compound $2 c$}

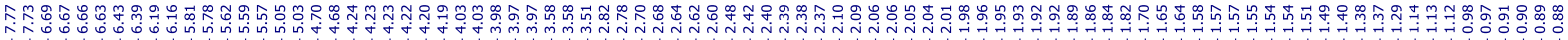

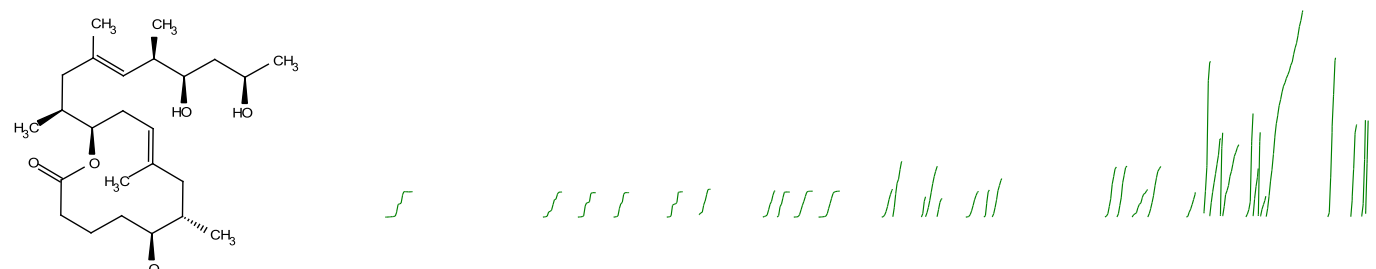<smiles>COC(=O)/C=C/C1=C(/C(C)=C/C=C/C(C)=C/C(O)[C@H](O)CC(C)C)CCC1</smiles>

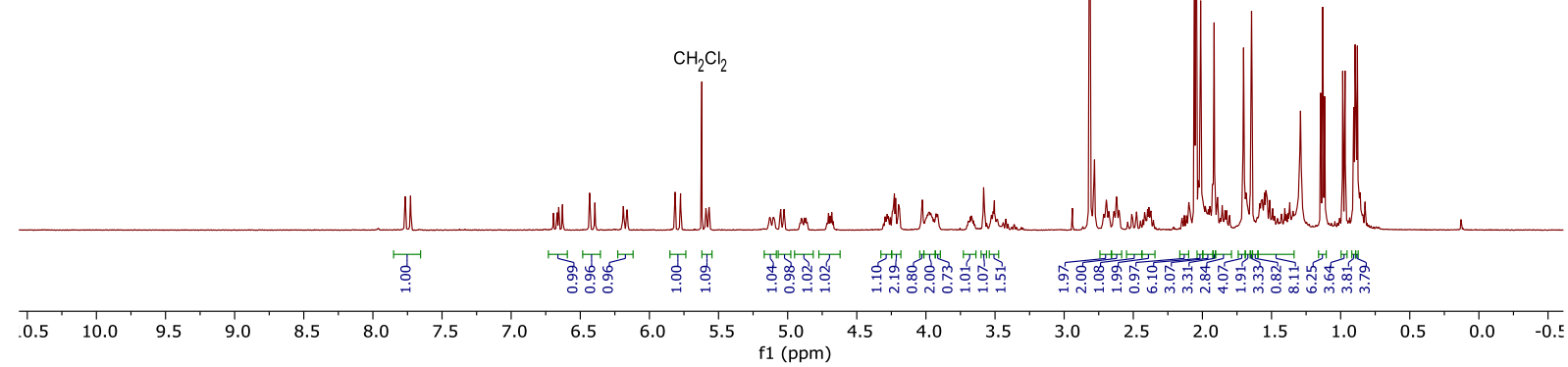

\section{${ }^{13} \mathrm{C}-\mathrm{NMR}$ of compound $2 \mathrm{c}$}

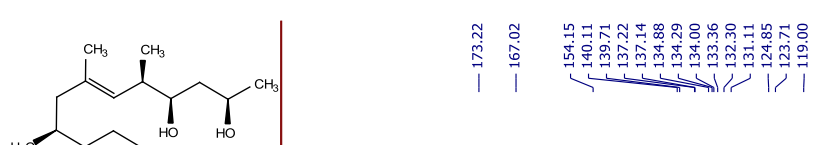

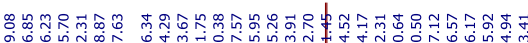

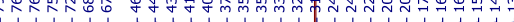

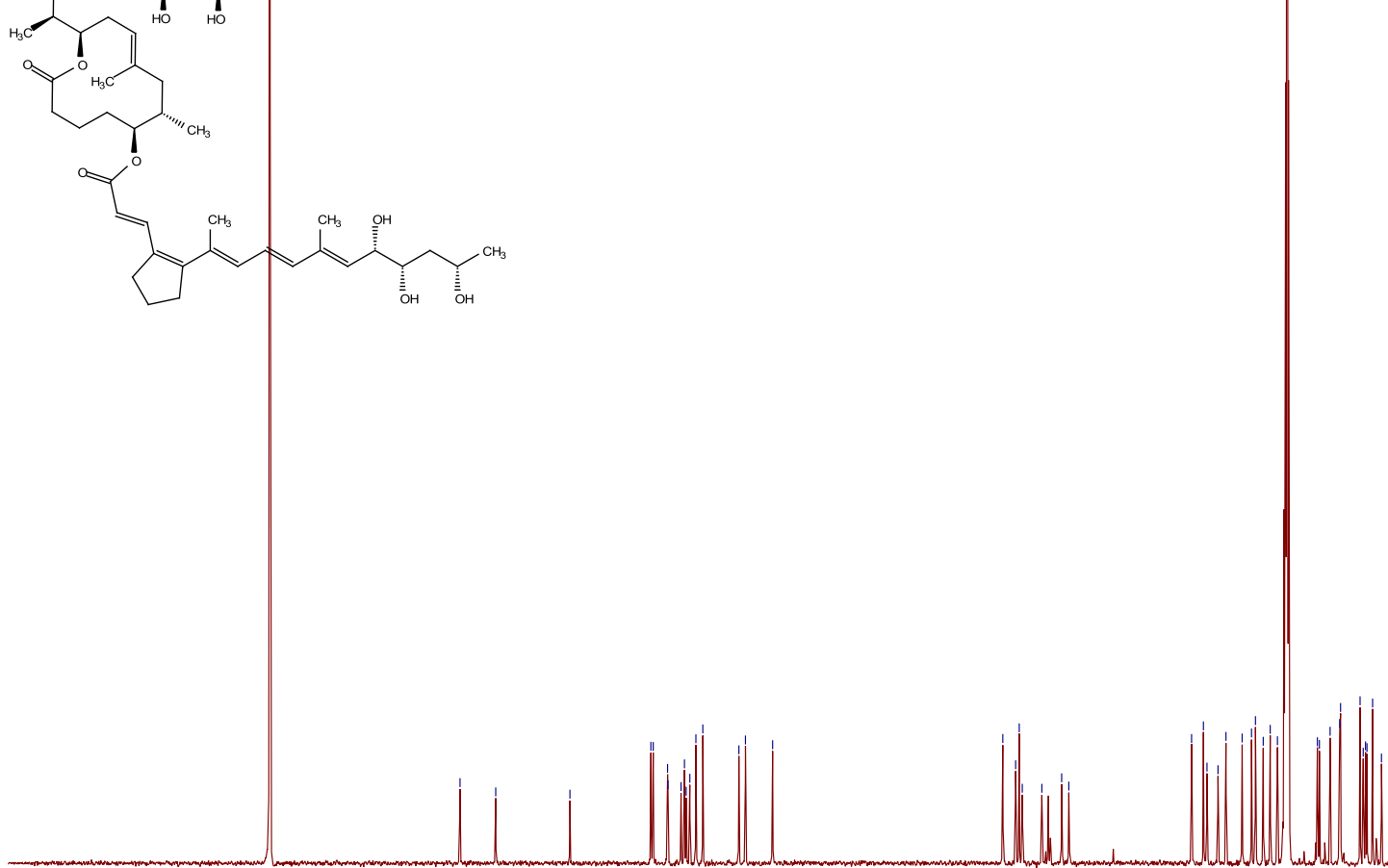

$\begin{array}{llllllllllllllllllllllllllll}150 & 240 & 230 & 220 & 210 & 200 & 190 & 180 & 170 & 160 & 150 & 140 & 130 & 120 & 110 & 100 & 90 & 80 & 70 & 60 & 50 & 40 & 30 & 20 & 10 & 0 & -\end{array}$ 